\title{
Spatially heterogeneous ages in glassy dynamics
}

\author{
Horacio E. Castillo ${ }^{1,2}$, Claudio Chamon ${ }^{1}$, Leticia F. Cugliandolo ${ }^{3,4,5}$, José Luis Iguain $^{4,6}$, Malcolm P. Kennett ${ }^{7,8}$ \\ ${ }^{1}$ Physics Department, Boston University, Boston, MA 02215, USA \\ 2 Department of Physics and Astronomy, Ohio University, Athens, OH 45701, USA \\ ${ }^{3}$ Laboratoire de Physique Théorique de l'École Normale Supérieure, Paris, France \\ ${ }^{4}$ Laboratoire de Physique Théorique et Hautes Energies, Jussieu, Paris, France \\ ${ }^{5}$ Lyman Laboratory of Physics, Harvard University, MA 02138, USA \\ 6 Département de Physique et Groupe de Recherche en Physique et Technologie des Couches Minces (GMC) \\ Université de Montréal, Case Postale 6128 Montréal, Québec H3H 3J7, Canada. \\ 7 Department of Physics, Princeton University, Princeton, New Jersey 08544, USA \\ 8 TCM Group, Cavendish Laboratories, Cambridge University, Madingley Rd, Cambridge CB3 OHE, UK
}

(November 19, 2018)

\begin{abstract}
We construct a framework for the study of fluctuations in the nonequilibrium relaxation of glassy systems with and without quenched disorder. We study two types of two-time local correlators with the aim of characterizing the heterogeneous evolution in these systems: in one case we average the local correlators over histories of the thermal noise, in the other case we simply coarse-grain the local correlators obtained for a given noise realization. We explain why the noise-averaged correlators describe the fingerprint of quenched disorder when it exists, while the coarse-grained correlators are linked to noise-induced mesoscopic fluctuations. We predict constraints on the distribution of the fluctuations of the coarse-grained quantities. In particular, we show that locally defined correlations and responses are connected by a generalized local out-of-equilibrium fluctuation-dissipation relation. We argue that large-size heterogeneities in the age of the system survive in the long-time limit. A symmetry of the underlying theory, namely invariance under reparametrizations of the time coordinates, underlies these results. We establish a connection between the probabilities of spatial distributions of local coarse-grained quantities and the theory of dynamic random manifolds. We define, and discuss the behavior of, a two-time dependent correlation length from the spatial decay of the fluctuations in the two-time local functions. We characterize the fluctuations in the system in terms of their fractal properties. For concreteness, we present numerical tests performed on disordered spin models in finite and infinite dimensions. Finally, we explain how these ideas can be applied to the analysis of the dynamics of other glassy systems that can be either spin models without disorder or atomic and molecular glassy systems.
\end{abstract}

PACS: 75.10.Nr, 75.10.Jm, 75.10.Hk, 05.30.-d

\section{INTRODUCTION}

A mean-field theory of glasses has been developed during the last two decades [1-3]. This approach is based on the study of fully-connected spin models with disorder. A fully-connected spin system with pairwise interactions, the Sherrington-Kirkpatrick (sK) model, is used to model spin-glasses [4-7]. Extensions in which the spins interact via all possible $p$-uplets, with $p \geq 3$, are used to describe structural glasses of fragile type $[8,9]$. These are the socalled $p$-spin models. Even though structural glasses consist of molecules moving in a finite dimensional volume, rather than spins interacting via random exchanges on a complete (hyper) graph, the disordered $p$-spin models yield a gross description of many important features of the structural glass phenomenology. For instance, they have dynamic and static transitions occurring at different values of the external temperature, mimicking the dynamic slowing down at the freezing temperature, $T_{g}$, and the entropy crisis at the Kauzmann temperature, $T_{s}$ [8]. More strikingly, these models capture the slow non-equilibrium dynamics characterized by macroscopic observables showing aging effects below $T_{g}[7,9]$.
Whilst they are successful in many respects, these models lack a geometric structure and hence cannot inform us about the spatial evolution of the glass former. In the context of spin-glasses, there is consensus about there being some kind of growing order below $T_{g}$. However, there has been a long-lasting debate about the characteristics of this order [10-20]. In other words, the question as to whether there are only two or many competing ground states, not related by symmetry, that grow during the nonequilibrium evolution $[12,20-24]$ has not been answered yet. In comparison, it is not even clear if there is a phase transition in structural glasses [25].

Very recently, a number of experiments have shown the appearance of mesoscopic regions in supercooled liquids and glasses that have distinctively different dynamics from the bulk of the system [26-30]. The position and identity of these "particles" changes in time. In general, these regions are referred to as dynamic heterogeneities and have also been identified in numerical simulations $[31,32]$.

Developing a theoretical description of the real-space dynamics of glassy systems is now a major challenge to theoreticians. The purpose of this paper is to expand on 
the theoretical framework we presented in Refs. [33,34] that allowed us to predict several properties of local dynamic fluctuations in spin-glasses. We test the predictions of our framework against numerical simulations on a spin model with disorder defined on a finite dimensional lattice, the Edwards-Anderson (EA) model $[4,5]$. Following the philosophy described in the first paragraph, we claim that the main results arising from our analysis will carry on rather simply to the particle models which are more relevant for real glasses. In the Conclusions and Perspectives sections we explain how one should translate our results and predictions to this case. We also list a number of glassy models on which our ideas could be tested.

\section{A. Background}

We first briefly summarize previous studies (mostly experimental and numerical) of dynamic heterogeneities in particle and spin systems that set the scene for our analysis.

There is great interest in the experimental observation of dynamic heterogeneous regions in super-cooled liquids and glasses. Many experimental techniques have been used to signal the existence of dynamically heterogeneous regions in samples of a variety of glasses [26-30], and to try to characterize their properties. The confocal microscopy technique $[28,29]$ is particularly useful for this, as it allows one to reconstruct the particle trajectories in $3 d$ space and have the complete configuration of the system at chosen times.

In the context of theoretical studies of structural and polymeric glasses, Bennemann et al. and Kob et al. identified fast moving particles embedded in a bulk of slow moving ones at temperatures above $T_{g}$ in the supercooled liquid phase of several models using molecular dynamics [31]. Other numerical studies of similar features appeared in [32].

In these experimental systems, and the models used to study them numerically, there is no quenched disorder. Moreover, the particles are identical and move in continuous space, so they cannot be identified by their position on a lattice, as in typical spin models. However, two possible ways of studying the heterogeneous dynamics of the system are as follows. (i) One can tag each particle, follow their evolution and detect which are the fast and slow moving particles during a previously chosen timewindow around some time after preparation. Particles are labeled by an index $i$. This is the route followed in Refs. [31,32]. (ii) One can divide the space into boxes of a chosen size and study the behavior of all particles within each box. The locality is then given by the position of the box which is labeled by $i$. At the end of this article we explain why we believe that the second approach will be very useful to characterize some spatial features of the nonequilibrium dynamics of glassy systems. An analysis of data obtained with the confocal microscopy technique of real systems and molecular dynamics of simple models along the lines described in this paper will yield valuable information for the future development of a complete analytic theory for glasses.

In the context of disordered spin models, fast and slow spins that decorrelate on totally different time-scales were identified in numerical simulations in Refs. [35] and [36] for the $3 d$ EA model above and below $T_{c}$, respectively. Ricci-Tersenghi and Zecchina [37] and Montanari and Ricci-Tersenghi [38] found a similar separation in the low temperature phase of spin models defined on random graphs. Even finite-size samples of mean-field models show important spin-to-spin fluctuations in the characteristic time-scale for individual decorrelation, as shown by Brangian and Kob for the disordered Potts model above its dynamic critical temperature [39]. A similar behavior, superposed on aging phenomena, is observed in the Sherrington-Kirkpatrick model at low temperatures, as shown in Sec. VIII B. In all these studies the noise-averaged correlations for fixed disorder were the focus of the studies. In this type of analysis the identity of a spin is determined by its position on the lattice or by the random exchanges.

In Refs. [33] and [34] we concentrated instead on the $3 d$ EA model and its two-time coarse-grained (but not noiseaveraged) local quantities. In Ref. [33] we showed that the action for the slow part of the local relaxation becomes reparametrization invariant asymptotically. This (approximate) symmetry allowed us to propose several properties of the dynamic behavior of the coarse-grained local correlations and responses that we tested numerically in Ref. [34]. These quantities are relevant both for spin models (with and without quenched disorder) and for continuous systems of interacting particles.

\section{B. Plan of the paper}

In this paper we complete the analysis that we started in Refs. [33] and [34]. We study several aspects of the local dynamics of the $2 d$ and $3 d$ EA spin-glass,

$$
\mathcal{H}_{J}=\sum_{\langle i, j\rangle} J_{i j} s_{i} s_{j}
$$

The sum runs over nearest neighbor sites $i, j$ on a $d$ dimensional cubic lattice. The couplings $J_{i j}$ take values $\pm J / \sqrt{2 z}$ with probability $1 / 2 . z$ is the coordination of the lattice, $z=2 d$ in the square/cubic case. The spins are Ising variables, $s_{i}= \pm 1$.

We also analyze the dynamic fluctuations in finite dimensional and fully-connected models with finite size. To test the latter we use the SK mean-field spin-glass model defined by 


$$
\mathcal{H}_{J}=\sum_{i \neq j} J_{i j} s_{i} s_{j}
$$

with $J_{i j}$ taken from a bimodal probability distribution with zero mean and variance $\left[J_{i j}^{2}\right]=J^{2} /(2 N)$. (We expect to find similar results using a Gaussian distribution of exchanges.) Here and in what follows we use square brackets to denote the average over disorder.

We fix the value of $J$ in such a way that the critical temperatures are at $T_{c} \sim 1.1$ for the $3 d$ EA model and at $T_{c}=1$ for the SK model. The $2 d$ EA model has a zero-temperature phase transition. We set the Boltzmann constant, $k_{B}$, to one.

We focus on two types of locally defined correlations and susceptibilities:

(i) coarse-grained local quantities $C_{i}^{c g}\left(t, t_{w}\right)$ and $\chi_{i}^{c g}\left(t, t_{w}\right)$.

(ii) noise-averaged local quantities $C_{i}^{n a}\left(t, t_{w}\right)$ and $\chi_{i}^{n a}\left(t, t_{w}\right)$.

The two time dependence reflects the out-of-equilibrium dynamics of these systems after the quench at time $t=0$. $t_{w}$ denotes the waiting-time elapsed after preparation and $t$ a longer time, $t \geq t_{w}$. We present a detailed comparison of the behavior of these local non-equilibrium correlations that have been averaged differently.

The numerical simulations were performed as follows. To study the finite $d$ EA model we evolved a cubic (square) system with side-length $L$ and periodic boundary conditions from a random initial condition using Metropolis dynamics at temperature $T$. The random initial configuration represents the result of an infinitely rapid quench from infinite temperature performed at $t=0$. In the $3 d$ case we considered $L=32$ and $L=64$ and for $d=2$ we considered $L=128$. To compute spatially coarse-grained two-time quantities we used a coarse-graining volume which is a cubic (square) box of linear size $2 M+1$. A coarse-graining time that serves to make the spin variable smooth varies from study to study and is noted in each plot. We considered several values of the external temperature that lie above and below $T_{c}$ and these are indicated as necessary. The noise-averaged data we present were obtained using shorter values of the waiting-time to allow for an average over many noiserealizations, typically $10^{3}$ samples.

When studying the SK model we evolved systems with $N=128$ and $N=512$ spins with Monte Carlo dynamics at $T=0.4$ also starting from a random configuration of spins. The noise-averaged data were obtained using, approximately, $10^{3}$ samples.

In short, the results in this paper are organized as follows:

First, we establish the dynamic scaling forms of both coarse-grained and noise-averaged local quantities numerically. In particular, we test scaling forms that we propose in Sec. II.
Second, we study the local relations between noiseaveraged correlation and integrated response and between the same quantities when coarse-grained.

Third, we show that the global quantities in finite size systems in finite dimensions and those defined on the complete graph show similar fluctuations as the local quantities in finite $d$ models.

Fourth, we propose a relation between the study of the probability distribution of local fluctuations and the theory of dynamic random surfaces.

Fifth, we define and analyze a dynamic correlation length that depends on two-times.

Sixth, we present a new way of looking at geometric properties in spin-glasses that should be relevant to future experiments with local probes. We analyze the realspace organization of local correlations, $C_{i}^{c g}$ and $C_{i}^{n a}$ by investigating the geometric properties of the (random) surfaces given by their evaluation on the substrate $d$ dimensional real space. In particular, we study the properties of clusters of spins with local correlation in the interval $[\mathrm{C}, \mathrm{C}+d \mathrm{C}]$ for which $\mathrm{C}$ is a parameter taking values between -1 and 1 . Again we compare the behavior of noise-averaged and coarse-grained quantities. A similar analysis could be applied to the random surface of local susceptibilities, $\chi_{i}^{c g}$ and $\chi_{i}^{n a}$.

Because real systems do not equilibrate on accessible time-scales, spatially resolved measurements will not be static; instead, they will still depend on the age of the system, very much like the bulk or global measurements. In these measurements one can monitor noise and response in a mesoscopic region of the sample. The results of our analysis will be of relevance to the interpretation and analysis of these experiments. They can also be used as a source of inspiration to analyze dynamic heterogeneities in super-cooled liquids and glasses $[27,31,32]$, as we discuss in the Conclusions and Perspectives sections.

\section{Summary of results}

Before getting into the technical details, let us summarize our results.

In Ref. [33] we showed that, in the limit of long-times, a zero mode develops in the dynamics of finite dimensional spin-glasses. This soft mode is related to the invariance of the effective action for the slow fields (that are actually two-time functions) under a global reparametrization of time. Thus, we argued that the least costly spatial fluctuations should be ones that smoothly change the local time reparametrization. In Ref. [34] we tested these ideas numerically by evaluating the local coarse-grained correlations and integrated responses in the $3 d \mathrm{EA}$ model. We observed that the fluctuations in these quantities are constrained to follow the fluctuation-dissipation relation (FDR) between the global quantities as a direct consequence of the existence of the asymptotic zero mode. 
In this paper we show further evidence that the coarsegrained two-time correlators reflect the existence of an asymptotic zero mode in the underlying theory. In particular, using the fact that the dynamics become "critical" in a well-chosen long time limit, we explain why a scaling limit of long-times and large coarse-graining volumes should exist in which the distributions of fluctuations approach a stable limit.

In disordered systems such as the $3 d$ EA model, another set of local two-time quantities can be defined using a different averaging procedure. Indeed, one can work with noise-averaged, as opposed to coarse-grained, two-time functions. Even if these quantities do not fluctuate in systems without quenched disorder, they do in spin-glasses and other random systems due to the fingerprint of disorder. One can then wonder if these quantities are also coupled to the asymptotic zero mode and whether their fluctuations are constrained in the same way as those of the coarse-grained quantities. We show numerically that this is not the case: the noise-averaged fluctuations behave in a rather different way.

In order to sustain further this claim, we also study the mesoscopic fluctuations in disordered models in finite and infinite dimensions. We show that the fluctuations in the global quantities, that are due to the finite size of the systems, behave just like the coarse-grained local quantities in finite dimensional models. We observed this property in the $3 d$ EA and the sK model.

We relate the study of the fluctuations in the local correlations (and susceptibilities) to the study of the evolution of random surfaces. Indeed, we propose that one can derive a "phenomenological" effective action for the fluctuations in the local quantities from the statistical analysis of the surfaces given by the evaluation of the two-time quantities on the $d$ dimensional substrate. This idea gives us a handle to describe analytically the fluctuations in a large variety of systems with slow dynamics.

We study numerically the random surfaces describing the fluctuations in space of both the coarse-grained and noise-averaged two-time local correlators. We show that noise-averaging leads to surfaces that encode the fingerprint of the disorder realization, and are static if the two times $t$ and $t_{w}$ have a fixed ratio (or $h(t) / h\left(t_{w}\right)$, with a more suitable function $h(t)$, is kept fixed).

The coarse-grained surfaces obtained for different pairs of times $\left(t, t_{w}\right)$, even if the ratio $t / t_{w}$ [or $\left.h(t) / h\left(t_{w}\right)\right]$ is fixed, fluctuate and cross each other at many points as a function of $t_{w}$. This result implies, as we show, that the relative age (as measured using the correlation value) between two sites $i$ and $j$ in the sample is not static, but fluctuates as a function of time. These are examples of sorpassi that we define in this paper, and show a clear contrast to noise-averaged local quantities, where the relative age between all sites in the sample keeps a constant, static, relative rank.

We define a two-time dependent correlation length $\xi\left(t, t_{w}\right)$ using the spatial correlation of the local two-time correlations. We study how this correlation length diverges asymptotically in the glassy phase of the $3 d$ EA model. We discuss how the ratio between the coarsegrained volume and the correlation length affects the probability distributions for the measured quantities. In particular, we argue that when the coarse-graining length is smaller than the correlation length, one probes the spatial fluctuations controlled by the zero mode. When using coarse-graining lengths that are larger, but still of the order of the correlation length one is measuring mesoscopic fluctuations of nearly independent finite size systems. If the coarse-graining length is much larger than the correlation length the fluctuations are suppressed. We show numerically that the qualitative features of the local and mesoscopic fluctuations are indeed very similar and we conjecture that they may have a similar origin.

Finally, we study the spatial organization of the local correlations (coarse-grained and noise-averaged). We propose that the analysis of the geometric properties of clusters of sites with similar values of the local two-time correlations can be useful to determine if one is at or below the lower critical dimension. More precisely, we claim that the geometric organization of the fluctuations in the $2 d$ EA model are different from those in the $3 d$ case signaling the fact that the former does not have a finite $T$ transition. The difference is very clear when one looks at the fractal dimension of clusters, $d_{f}$. In 3 dimensions $d_{f} \sim 2$, whilst in 2 dimensions, $d_{f} \leq 2$. These values of the fractal dimension are quite close to each other - however, the difference between the fractal dimension and that of the substrate space, $\Delta=d-d_{f}$, is very different. In 3 dimensions, $\Delta \sim 1$ and in 2 dimensions $\Delta \ll 1$. This suggests that the level surfaces in 2 dimensions lie on a much rougher underlying manifold, as we suggest from theoretical arguments in Sec. III D, and should be linked to the absence of a glass transition in $d=2$. However, a multifractal analysis gives less clear-cut distinctions, suggesting that the non-equilibrium aging regime in the 2 dimensional case has aspects that are very similar to the 3 dimensional case.

In the conclusions we discuss how to adapt this approach and the picture that emerges to super-cooled liquids and structural glasses.

\section{DEFINITIONS AND DISCUSSION}

In this section we define the two-time, global and local, correlators that we study numerically in the rest of the paper. We recall some known properties of the global correlators. We then discuss possible scaling forms for the local quantities, as well as the implications of these scaling forms. 


\section{A. Two-time dependent global functions}

To date, analytical, numerical, and experimental studies in glassy systems have mainly focused on the global correlation and integrated response:

$$
\begin{aligned}
C\left(t, t_{w}\right) & \equiv \frac{1}{N} \sum_{i=1}^{N} s_{i}(t) s_{i}\left(t_{w}\right), \\
\chi\left(t, t_{w}\right) & \left.\equiv \frac{1}{N} \sum_{i=1}^{N} \int_{t_{w}}^{t} d t^{\prime} \frac{\delta s_{i}(t) \epsilon_{i}}{\delta \eta_{i}\left(t^{\prime}\right)}\right|_{\eta=0},
\end{aligned}
$$

where $\eta_{i}$ is a (site-dependent) magnetic field given by $\eta_{i}=\eta \epsilon_{i}$ with $\epsilon_{i}= \pm 1$ with probability $\frac{1}{2}$ and $\eta$ its magnitude. The field couples linearly to the spin, $H_{J} \rightarrow$ $H_{J}-\sum_{i} s_{i} \eta_{i}$. The product $s_{i}(t) \epsilon_{i}$ is the "staggered local spin", i.e. the projection of $s_{i}$ on the direction of the local external field $\eta_{i}$. In order to extract the linear part of the response the variation is evaluated at zero field, $\eta=0$. The integrated response is usually averaged over many realizations of the random field.

For an infinite system that evolves out of equilibrium these quantities are self-averaging, and thus averages over the thermal noise and disorder (if existent) are not required. All the generic analytic arguments we shall develop assume that the thermodynamic limit, $N \rightarrow \infty$, has been taken at the outset. (We discuss finite size effects in Sec. VIII.)

\section{B. Two-time dependent local functions}

Quenched random interactions have a strong effect on the local properties of spin systems. For instance, Griffiths singularities in the free-energy of random ferromagnets are due to regions in space with strong ferromagnetic couplings [40]. These lead to dynamic slowing down even in the disordered phase of the random problem, below the transition temperature of the pure model. It is natural to expect that heterogeneous dynamics in spin-glasses arises for similar reasons. In these systems random exchanges can be very different between one region of the sample and another: some regions can have purely ferromagnetic interactions, others can have purely antiferromagnetic ones, others can be frustrated. One can analyze the fingerprint of the disorder on the local dynamics by choosing not to average over the random exchanges.

However, heterogeneous dynamics do not arise simply because of quenched random couplings. Glassy systems with no explicit quenched disorder also exist in nature [25]. Many models with spin or particle variables that capture their behavior have been proposed [41-43]. Even if there is no quenched disorder in these systems, one expects to find heterogeneous dynamics in which some regions evolve differently than others $[30,43]$.
An extreme example of the latter situation occurs in ferromagnetic domain growth [44]. At any finite time with respect to the size of the system, a coarsening system is heterogeneous. Observed on a very short timewindow, spins on interfaces behave very differently from spins in the bulk of domains. However, there is nothing special about the identity of these spins. Spins that belong to an interface at one time can later become part of a domain and even later be part of another wall. Importantly, no local region (not even of the minimum linear size given by the lattice spacing) can be considered to be equilibrated while coarsening is taking place.

If one wishes to analyze the local fluctuations in the dynamics of spin systems, two natural functions to monitor are the two-time local correlations and responses. These can be made continuous (in Ising spin systems) through different averaging procedures that highlight different properties of the systems. Each definition has a different theoretical motivation.

First, consider spatially coarse-grained functions [34]

$$
\begin{aligned}
C_{i}^{c g}\left(t, t_{w}\right) & \equiv \frac{1}{V} \sum_{j \in V_{i}} \bar{s}_{j}(t) \bar{s}_{j}\left(t_{w}\right), \\
\chi_{i}^{c g}\left(t, t_{w}\right) & \left.\equiv \frac{1}{V} \sum_{j \in V_{i}} \int_{t_{w}}^{t} d t^{\prime} \frac{\delta \bar{s}_{j}(t) \epsilon_{j}}{\delta \eta_{j}\left(t^{\prime}\right)}\right|_{\eta=0},
\end{aligned}
$$

where $V_{i}$ is a coarse-graining region centered on site $i$ with volume $V$, and the overline stands for a coarse-graining over a short time-window $\tau_{t}\left(\tau_{t_{w}}\right)$ around $t\left(t_{w}\right), \tau_{t} \ll t$ $\left(\tau_{t_{w}} \ll t_{w}\right)$. (Note that we use the same coarse-graining volume on all sites.) Only one realization of the thermal noise is used here, which mimics nature. This definition is natural for the study of finite dimensional models in which there is a notion of space and neighborhood. Indeed, a coarse-graining procedure of this type is usually used to derive a continuum field-theoretical description of a problem originally defined on a lattice [45]. Moreover, it is of use if one wants to compare the behavior of finite dimensional models with and without disorder since it is non-trivial in both cases. This quantity is also relevant to compare with experiments in which mesoscopic probes are limited to testing the behavior of regions with a minimum size that involve a large (though mesoscopic) number of spins.

Second, one can define single-site noise-averaged functions $[35-39,46]$ :

$$
\begin{aligned}
C_{i}^{n a}\left(t, t_{w}\right) & \equiv\left\langle\bar{s}_{i}(t) \bar{s}_{i}\left(t_{w}\right)\right\rangle, \\
\chi_{i}^{n a}\left(t, t_{w}\right) & \left.\equiv \int_{t_{w}}^{t} d t^{\prime} \frac{\delta\left\langle\bar{s}_{i}(t) \epsilon_{i}\right\rangle}{\delta \eta_{i}\left(t^{\prime}\right)}\right|_{\eta=0} .
\end{aligned}
$$

Here and in what follows the angular brackets represent the average over thermal histories. These functions will appear, for example, in a dynamic cavity method applied to a disordered model [47]. This definition is particularly 
useful for mean-field (fully-connected and dilute) systems with quenched disorder for which there is no notion of neighborhood. However, it completely erases the inherent heterogeneity of the dynamics in a non-disordered system such as ferromagnetic domain growth. Moreover, a single-spin experimental measurement is unlikely, and usually a region involving a large number of spins is probed, implying an effective coarse-graining.

Neither of the two definitions above include, in the case of random systems, an average over disorder realizations. This may allow us to detect regions that have special behavior due to the random interactions. We insist on the fact that the coarse-grained definition still contains noise-induced fluctuations.

\section{Correlation-scales}

The relaxation of glassy systems may take place on many different time scales. A precise definition of "correlation-scales" was given in [7]. Assuming that a chosen two-time correlation, $C$, is a monotonic function of both times $t$ and $t_{w}$, in the long waiting-time limit, one can relate the values it takes at any three times using a time-independent function. More precisely, $C\left(t_{1}, t_{3}\right)=f\left[C\left(t_{1}, t_{2}\right), C\left(t_{2}, t_{3}\right)\right]$ for $t_{1} \geq t_{2} \geq t_{3}$ when all three times are very long.

The correlation scales are defined as follows. Within a correlation scale, $f(x, y)=\jmath^{-1}[\jmath(x) \jmath(y)]$ and $C\left(t_{1}, t_{2}\right)=$ $\jmath^{-1}\left[h\left(t_{1}\right) / h\left(t_{2}\right)\right]$ with $h(t)$ a monotonic function of time and $\jmath(x)$ another function. Between correlation-scales the function $f$ is "ultrametric", $f(x, y)=\min (x, y)$.

To explain this definition with an example, the correlation function $C\left(t, t_{w}\right)=\left(1-q_{\mathrm{EA}}\right) \exp \left[-\left(t-t_{w}\right) / \tau\right]+$ $q_{\mathrm{EA}}\left(t_{w} / t\right)$ decays in two scales that are separated at the value $C=q_{\mathrm{EA}}$ that one sees as a plateau in $C$ that develops at long $t_{w}$ in a plot against $t-t_{w}$ in logarithmic scale. The first scale is stationary and characterized by $h_{\mathrm{ST}}(t)=\exp (-t / \tau)$, the second one ages and is characterized by $h_{\mathrm{AG}}(t)=t$.

The structure of scales can be different for different correlators. The local correlations defined in Eq. (5) [or in Eq. (7)] are different observables labelled by $i$. Their decay should follow these generic rules whenever one can assume that they are monotonic.

\section{Behavior of global two-time quantities}

In the long waiting-time limit, taken after the thermodynamic limit, one can prove analytically that a sharp separation of time-scales characterizes the dynamics of mean-field glassy models [7,9,48-50]. A similar separation of time-scales has been observed numerically [51-54] and experimentally [21] in a variety of glassy systems. In short, one finds: [55] (i) a fast stationary evolution at short time-differences in which the correlation approaches a plateau defined as the Edwards-Anderson parameter

$$
q_{\mathrm{EA}} \equiv \lim _{t-t_{w} \rightarrow \infty} C_{\mathrm{ST}}\left(t-t_{w}\right) \equiv \lim _{t-t_{w} \rightarrow \infty} \lim _{t_{w} \rightarrow \infty} C\left(t, t_{w}\right),
$$

with the integrated response linked to the correlation by the fluctuation-dissipation theorem (FDT)

$$
\begin{aligned}
\chi_{\mathrm{ST}}\left(t-t_{w}\right) & \equiv \lim _{t_{w} \rightarrow \infty} \chi\left(t, t_{w}\right) \\
& =\frac{1}{T}\left[1-C_{\mathrm{ST}}\left(t-t_{w}\right)\right],
\end{aligned}
$$

and, in particular,

$$
\lim _{t-t_{w} \rightarrow \infty} \lim _{t_{w} \rightarrow \infty} \chi\left(t, t_{w}\right)=\frac{1}{T}\left(1-q_{\mathrm{EA}}\right) .
$$

This regime is also called time-translational invariant (TTI). In general, the decay in this regime is not exponential ( $c f r$. the example given in Sec. II C).

(ii) A slow aging relaxation for longer time-differences

$$
\begin{aligned}
& C\left(t, t_{w}\right) \neq C\left(t-t_{w}\right), \\
& \chi\left(t, t_{w}\right) \neq \chi\left(t-t_{w}\right),
\end{aligned}
$$

when the value of the global correlation drops below $q_{\mathrm{EA}}$ [and the integrated response goes above $\left(1-q_{\mathrm{EA}}\right) / T$ ].

The number of scales that appear in this second decay depends on the model considered. For the fullyconnected $p$ spin model a single correlation-scale has been found below $q_{\mathrm{EA}}$ in which the global correlation scales with a power law, $h_{\mathrm{AG}}(t)=t[9,56]$. A sequence of globalcorrelation scales exists in the analytic solution to the SK model $[7,48]$. For a manifold moving in an infinite dimensional embedding space with a short-ranged random potential one finds that the Fourier modes of the correlation $C_{r}\left(t, t_{w}\right)=\int_{V} d^{d} r^{\prime}\left\langle\vec{\phi}\left(\vec{r}+\vec{r}^{\prime}, t\right) \vec{\phi}\left(\vec{r}^{\prime}, t_{w}\right)\right\rangle$ decay on two scales that are separated at a $k$-dependent EdwardsAnderson value, $q_{\mathrm{EA}}^{k}[49]$. The functions $h_{\mathrm{ST}, \mathrm{AG}}$ that characterize the two scales are identical for all wave-vectors. This has also been found in molecular dynamic simulations of Lennard-Jones mixtures [53,54]. If the manifold feels a a long-ranged random potential $[49,50]$ the $k$-modes decay in a sequence of scales. If this structure exists in any non-mean-field problem it is not clear yet. In particular, the $3 d$ EA model behaves more like a model with only two global-correlation scales [51] but this may be due to the shortness of the simulation times. In more complex problems one could even find that different correlators decay on totally different time scales.

In the second time regime the FDT is not satisfied. However, in many glassy models evolving out of equilibrium the global correlation [Eq. (3)] and the global integrated response [Eq. (4)] are linked in a rather simple manner [7]. Indeed, assuming that the global correlation decays monotonically as a function of $t$, one can invert this function and write $t=\tilde{f}_{g}^{-1}\left(C\left(t, t_{w}\right), t_{w}\right)$ and 


$$
\chi\left(t, t_{w}\right)=\chi\left(\tilde{f}_{g}^{-1}\left(C\left(t, t_{w}\right), t_{w}\right), t_{w}\right),
$$

implying

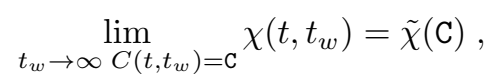

where $\mathrm{C}$ is held fixed as we take the limit which we assume exists. We call Eq. (14) a fluctuation-dissipation relation (FDR). In equilibrium $\tilde{\chi}(\mathrm{C})=(1-\mathrm{C}) / T$ holds for all values of $\mathrm{C}$. Out of equilibrium one finds a different relation with a kink: when $\mathrm{C}>q_{\mathrm{EA}}$ the equilibrium result holds, $\tilde{\chi}(\mathrm{C})=(1-\mathrm{C}) / T$; when $\mathrm{C}<q_{\mathrm{EA}}$ a nontrivial functional form $\tilde{\chi}(\mathrm{C})$ is found and its particular form depends on the model or system considered. For disordered spin models of the mean-field type two types of curves have been found:

(i) For $p$ spin models it is a broken line with two different slopes, one being the negative of the inverse temperature of the bath for $\mathrm{C} \in\left[q_{\mathrm{EA}}, 1\right]$; the other has a different slope and spans the interval $\mathrm{C} \in\left[0, q_{\mathrm{EA}}\right][9,56]$.

(ii) For the SK model the construction also has a breaking point at $q_{\mathrm{EA}}$ separating a straight line with slope minus the inverse temperature of the bath for $\mathrm{C} \in\left[q_{\mathrm{EA}}, 1\right]$, and a curved piece for $\mathrm{C} \in\left[0, q_{\mathrm{EA}}\right][7]$.

In the case of the random manifold the form of the modifications of FDT depend on the range of correlation of the random potential [49]. One finds a linear relation between the Fourier modes of the space-dependent correlator and susceptibility if it is short-ranged and a non-linear form if it is long-ranged. Moreover, one finds that all modes behave essentially in the same way in both cases. We shall discuss this issue in Sec. IIF.

Several simulations support the fact that the FDR of global quantities in models for structural glasses, such as Lennard-Jones mixtures, behave like a manifold in a short-range correlated random potential, for which the second slope does not vanish and is equal for all the wavevectors $k[53,54]$. In the case of the $3 d$ EA model the numerical results were interpreted as supporting the existence of a curve with non-constant slope below $q_{\mathrm{EA}}$ [57]. Similar conclusions were drawn from the experimental work presented in [58]. The numerical and experimental data are still rather far from the asymptotic time limit, and, in our opinion, it is quite difficult to decide from the present data if $\tilde{\chi}(C)$ is indeed curved or a straight line below $q_{\mathrm{EA}}$.

\section{E. Behavior of local correlations}

In this section we denote by $C_{i}$ a generic local correlation that has been coarse-grained, noise-averaged, or smoothed with any other prescription. We first discuss the possible time dependences of individual correlations at a very general level. Later we distinguish between coarse-grained and noise-averaged values.

\section{Local Edwards-Anderson parameter}

Similarly to our discussion of the decay of the global correlation, see Eq. (9), one can define a local EdwardsAnderson parameter as the value of the local correlation separating fast and slow decays,

$$
q_{\mathrm{EA}}^{i} \equiv \lim _{t-t_{w} \rightarrow \infty} \lim _{t_{w} \rightarrow \infty} C_{i}\left(t, t_{w}\right) .
$$

The local correlations should decay quickly towards $q_{\text {EA }}^{i}$ and then slowly below this value. If the structure of the global correlation is preserved at the local level, the first step of the relaxation should be stationary whilst the second one could be waiting-time dependent.

Site-to-site fluctuations in $q_{\mathrm{EA}}^{i}$ are possible. For some spins the local Edwards-Anderson parameter may vanish, i.e. $q_{\mathrm{EA}}^{i}=0$, and they may only show the first, fast decay. These are the fast spins identified in Refs. [36-38] in purely noise averaged local correlations. However, one expects the fluctuations in $q_{\mathrm{EA}}^{i}$ to be smoothed by spatial coarse-graining and to disappear if a sufficiently large coarse-graining volume $V$ is used [34].

\section{Slow local relaxation}

What about the decay of the local correlations below the plateau at $q_{\mathrm{EA}}^{i}$ ? Any monotonically decaying two-time correlation within a correlation-scale behaves as [7]

$$
C_{i}\left(t, t_{w}\right)=f_{i}\left(\frac{h_{i}(t)}{h_{i}\left(t_{w}\right)}\right) .
$$

There are two special cases that deserve special mention. On the one hand, the scaling arguments $h_{i}(t)$ could be site-independent, $h_{i}(t)=h(t)$, and thus

$$
C_{i}\left(t, t_{w}\right)=f_{i}\left(\frac{h(t)}{h\left(t_{w}\right)}\right) .
$$

On the other hand, the external function $f_{i}$ could be independent of the site index $i$ and the scaling could take the form

$$
C_{i}\left(t, t_{w}\right)=f\left(\frac{h_{i}(t)}{h_{i}\left(t_{w}\right)}\right) .
$$

This allows several combinations. The main ones to be discussed below are: (i) the scaling in Eq. (17) holds for all sites in the sample, (ii) the scaling in Eq. (18) holds everywhere in the sample. Evidently, one can also have more complicated behaviors: (iii) parts of the sample scale as in Eq. (17), other parts scale as in Eq. (18), and still other parts do not satisfy either of the special forms but are described by the more general form (16). Note that the noise-averaged and coarse-grained local correlations do not necessarily scale in the same way. 
We first explore the consequences of having the behavior in Eq. (17) for all sites in the sample. For simplicity, let us first assume that the global correlation decays in a single correlation-scale once its value drops below the global Edwards-Anderson parameter,

$$
C\left(t, t_{w}\right)=f_{g}\left(\frac{h_{g}(t)}{h_{g}\left(t_{w}\right)}\right), \quad C<q_{\mathrm{EA}} .
$$

This is the behavior of fully-connected $p$-spin models with $p \geq 3[9]$. One can expect it to hold in dilute ferromagnetic $p=3$ spin models [38] and, surprisingly, it is also suggested by numerical simulations in the $3 d$ EA model, at least for the waiting and total times explored [51]. If the hypothesis in Eq. (19) holds, the $h(t)$ in Eq. (17) must be identical to $h_{g}(t)$, and one can write

$$
C_{i}\left(t, t_{w}\right)=f_{i}\left(f_{g}^{-1}\left(C\left(t, t_{w}\right)\right)\right)=\tilde{f}_{i}\left(C\left(t, t_{w}\right)\right) .
$$

For all pairs of times $\left(t, t_{w}\right)$ leading to a fixed value of the global correlation, $C\left(t, t_{w}\right)=\mathrm{C}$, one has

$$
C_{i}\left(t, t_{w}\right)=\tilde{f}_{i}(\mathrm{C})
$$

All local correlations are then site-dependent functions of the global correlation. Following the line of thought in Ref. [7] one can extend this argument to the case with a global correlation that decays over many time scales. If one assumes that the global correlation is monotonic, one can write $C_{i}$ as a function of $C\left(t, t_{w}\right)$ and $t_{w}$ using $t=\tilde{f}_{g}^{-1}\left(C\left(t, t_{w}\right), t_{w}\right)$. If one further assumes that in the limit $t_{w} \rightarrow \infty$ with $C\left(t, t_{w}\right)=\mathrm{C}$ fixed each local correlation approaches a limit, then

$$
\lim _{t_{w} \rightarrow \infty, C\left(t, t_{w}\right)=\mathrm{C}} C_{i}\left(t, t_{w}\right)=\tilde{f}_{i}(\mathrm{C}) .
$$

This means that within each correlation scale all local correlations are locked into following the scaling of the global one.

Replacing the local space index $i$ by a wave vector $k$, this is exactly the behavior of a finite dimensional manifold embedded in an infinite dimensional space [49]. When the random potential is short-range correlated the dynamics are such that there are only two correlation scales in the problem, a TTI one satisfying FDT and an aging one in which all $k$-dependent correlators decay below $q_{\mathrm{EA}}^{k}$ as in Eq. (17). Instead, when the random potential is of long-range type, the correlations have a TTI scale that ends at a $k$-dependent Edwards-Anderson plateau, $q_{\mathrm{EA}}^{k}$, and a subsequent decay that takes place in a sequence of scales. All modes are locked in the sense that for all times one can write $C_{k}=f_{k}\left(C_{0}\right)$ where the argument can be any chosen mode, for instance, the $k=0$ one. The former behavior was also found numerically in the $k$ modes of the incoherent scattering function of a Lennard-Jones mixture where there is only one correlation scale below the $k$-dependent $q_{\mathrm{EA}}^{k}$ [53]. Recently it was also proposed for all site dependent correlators in the context of a ferromagnetic model on a random graph in Ref. [38] and for the $3 d$ EA model in Ref. [46]. In these cases the correlations and susceptibilities were averaged over the noise with no spatial coarse graining.

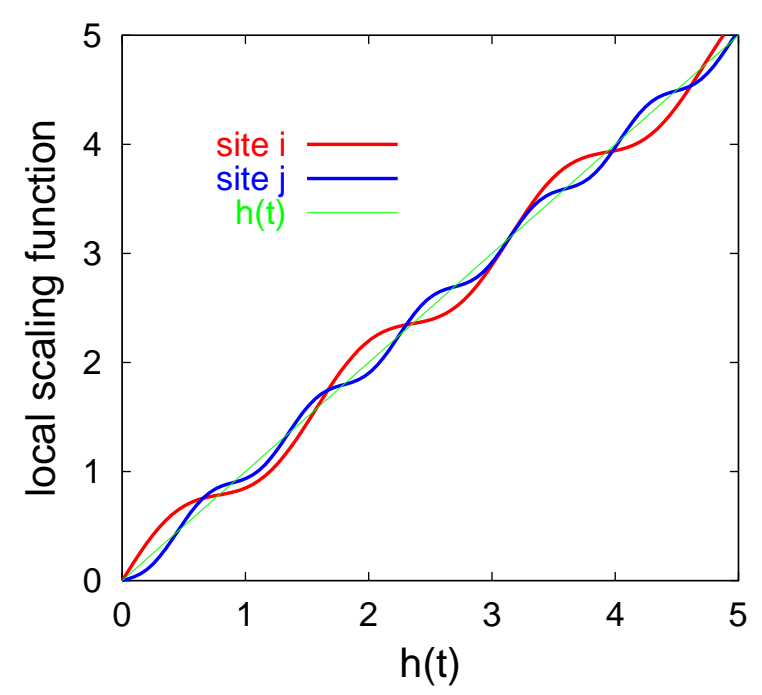

FIG. 1. Example of scaling functions $h_{i, j}(t) v s$. $h(t)$. Because the functions $h_{i, j}(t)$ cross, there are pairs of times $\left(t, t_{w}\right)$ for which $C_{i}>C_{j}$, but others for which $C_{i}<C_{j}$, even though $h_{i, j}(t)$ only fluctuate slightly around the scaling function $h(t)$.

Instead, if the hypothesis in Eq. (18) holds, there are many open possibilities. For instance, different sites can evolve on totally different time-scales. A simple example illustrating this point is given by two sites $i$ and $j$ that decay below their Edwards-Anderson values to zero in only one scale but that are different: $C_{i}\left(t, t_{w}\right)=$ $q_{\mathrm{EA}}^{i}\left(\ln t_{w}\right) /(\ln t)$ and $C_{j}\left(t, t_{w}\right)=q_{\mathrm{EA}}^{j} t_{w} / t$. In this case, site $i$ decays on a slower scale than site $j$. There is also the possibility that, while being in the same correlation scale, the value of the correlations at two sites $i, j$ may cross each other as a function of time. Let us be more explicit; consider two sites with local scaling functions

$$
\begin{aligned}
& h_{i}(t)=h(t)+\delta h_{i}(t), \\
& h_{j}(t)=h(t)+\delta h_{j}(t),
\end{aligned}
$$

with $\left|\delta h_{i, j}(t)\right| \ll h(t)$, so that the sites evolve within the same scale. [Note that both $h_{i}(t)$ and $h_{j}(t)$ are monotonic functions.] In this case, one can expand the expression in Eq. (18) and write:

$$
\begin{aligned}
C_{i}\left(t, t_{w}\right) \approx & f\left[\frac{h(t)}{h\left(t_{w}\right)}\left(1+\frac{\delta h_{i}(t)}{h(t)}-\frac{\delta h_{i}\left(t_{w}\right)}{h\left(t_{w}\right)}\right)\right] \\
\approx & f\left(\frac{h(t)}{h\left(t_{w}\right)}\right) \\
& +f^{\prime}\left(\frac{h(t)}{h\left(t_{w}\right)}\right) \frac{h(t)}{h\left(t_{w}\right)}\left(\frac{\delta h_{i}(t)}{h(t)}-\frac{\delta h_{i}\left(t_{w}\right)}{h\left(t_{w}\right)}\right),
\end{aligned}
$$


and similarly for $C_{j}\left(t, t_{w}\right)$. At any pair of times $\left(t, t_{w}\right)$, the difference between the two local correlations is

$$
C_{i}-C_{j} \propto \frac{\delta h_{i}(t)-\delta h_{j}(t)}{h(t)}-\frac{\delta h_{i}\left(t_{w}\right)-\delta h_{j}\left(t_{w}\right)}{h\left(t_{w}\right)} .
$$

In general, the right-hand-side (r.h.s.) of Eq. (25) can change sign as a function of the two times $t$ and $t_{w}$, allowing for sorpassi (passing events) between different sites. An example of functions $h_{i}$ and $h_{j}$ for which these sorpassi are realized is shown in Fig. 1.

Finally, one could also find cases in which $\delta h_{i, j}(t)$ are large $\left[\left|\delta h_{i, j}(t)\right| \ll h(t)\right.$ is not satisfied] and the sorpassi can be such that $C_{i}$ and $C_{j}$ move across correlation scales. These variations are quite dramatic. One could expect them to be penalized in such a way that they appear less frequently than the ones in which $\left|\delta h_{i, j}(t)\right| \ll h(t)$. We shall return to this issue in Sec. III.

\section{F. Behavior of local responses and fluctuation-dissipation relation}

Let us define a generalized local fluctuation-dissipation relation (FDR) via the limit

$$
\lim _{t_{w} \rightarrow \infty} \chi_{C_{i}\left(t, t_{w}\right)=\mathrm{C}_{i}} \chi_{i}\left(t, t_{w}\right)=\tilde{\chi}_{i}\left(\mathrm{C}_{i}\right) .
$$

This limit exists whenever the local correlations are not multivalued, i.e. if they are monotonic functions of $t$ for fixed $t_{w}$. Indeed, in this case one can invert for the time

$$
t=\tilde{f}^{-1}\left(C_{i}\left(t, t_{w}\right), t_{w}\right)
$$

and write

$$
\chi_{i}\left(t, t_{w}\right)=\chi_{i}\left(\tilde{f}^{-1}\left(C_{i}\left(t, t_{w}\right), t_{w}\right), t_{w}\right) .
$$

Taking the limit $t_{w} \rightarrow \infty$ while keeping $C_{i}\left(t, t_{w}\right)$ fixed to $\mathrm{C}_{i}$ one recovers Eq. (26).

The question now arises as to whether the fluctuations in $C_{i}$ and $\chi_{i}$ are independent or whether they are constrained to satisfy certain relations.

Based on thermometric arguments [59], we shall associate the variation of $\tilde{\chi}_{i}$ with respect to $C_{i}$ to a local effective-temperature. We would also like to know if the values of the local effective-temperatures are constrained or can freely fluctuate.

In the rest of this subsection we discuss different scenarios for the behavior of the joint probability distribution (PDF) $\rho\left(C_{i}, \chi_{i}\right)$ computed at two fixed times $t$ and $t_{w}$. We also discuss the behavior of the fluctuations in the local effective-temperature. In order to illustrate different possibilities we present several plots that sketch the following construction. Given a pair of times $t_{w} \leq t$, we depict the $N$ points $\left(C_{i}\left(t, t_{w}\right), T \chi_{i}\left(t, t_{w}\right)\right)$ with arrows that represent the velocity of the points $[i . e$, the rate at which the $\left(C_{i}, T \chi_{i}\right)$ positions change as one changes $\left.t\right]$, and are located at their position in the $C-T \chi$ plane. Note that all these points are evaluated at the same pair of times $\left(t, t_{w}\right)$. For the sake of comparison, in the same plots we also draw the parametric plot for the global $T \tilde{\chi}(C)$ constructed as usual [7]: for a fixed $t_{w}$ we follow the evolution of the pair $(C, T \chi)$ as time $t$ evolves from $t=t_{w}$ to $t \rightarrow \infty$.

In the figures showing the distributions of $\left(C_{i}, \chi_{i}\right)$ pairs we scale the $\mathrm{y}$-axis by temperature to work with dimensionless variables. This is important to compare the extent of fluctuations in the two directions.

\section{Similar times}

Let us first discuss the case in which the two times $t$ and $t_{w}$ chosen to evaluate the local correlation and integrated linear response are close to each other, in such a way that the global correlation between them lies above the Edwards-Anderson parameter. In this case the global correlator and the global linear integrated response are stationary and related by the FDT. In this regime of times we also expect the local quantities to be linked by the FDT.

When studying noise-averaged two-time quantities one can actually use the bound derived in Ref. [60] to justify this claim. For coarse-grained quantities we do not have an analytic argument to use but we find this expectation very reasonable. Note that the arguments put forward in Sec. III do not apply to these short time-differences since they hold for the slowly varying part of the two-time functions only. We have verified numerically that the fluctuations of the local coarse-grained correlations and responses are concentrated rather spherically around the global value $\chi=(1-C) / T$ when $C>q_{\mathrm{EA}}[34]$.

\section{Far away times: no constraint}

We now choose two times $t$ and $t_{w}$ such that the global correlation taken between them is less than $q_{\mathrm{EA}}$.

The simplest possibility is that the fluctuations in $C_{i}$ and $\chi_{i}$ are independent. If these are not specially constrained the pairs $\left(C_{i}, T \chi_{i}\right)$ can be scattered almost everywhere on the $C-T \chi$ plane.

The scaling in Eq. (16) means that different sites might evolve on different time-scales. This could happen, for example, if $h_{i}(t)=t$ and $h_{j}(t)=\ln \left(t / t_{0}\right)$. Hence, one can expect each site to have their own effective temperature,

$$
-\beta_{i}^{\mathrm{EFF}}\left(C_{i}\right) \equiv \frac{d \tilde{\chi}_{i}\left(C_{i}\right)}{d C_{i}}
$$

This situation is sketched in Fig. 2. 


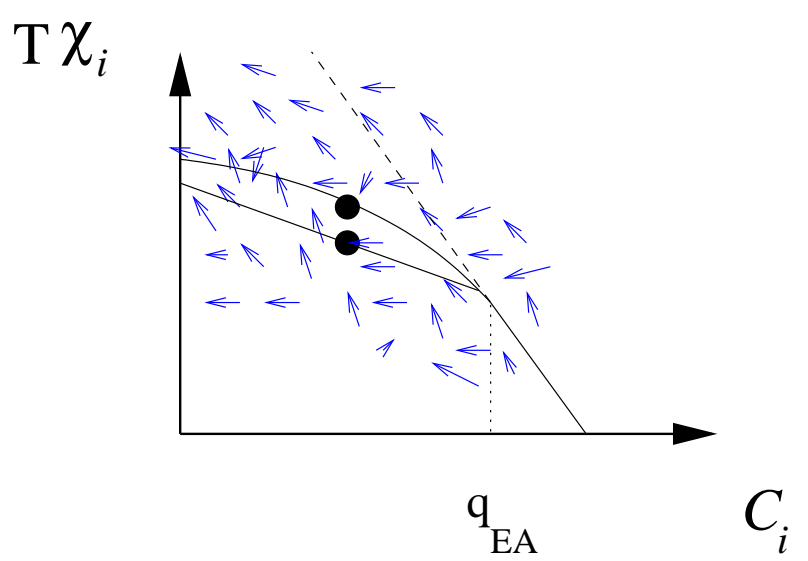

FIG. 2. Sketch of the local FDR between integrated responses and correlations on all sites, at a fixed pair of times $\left(t, t_{w}\right)$ such that the global correlation goes below $q_{\mathrm{EA}}$. In this case neither the positions of the pairs $\left(C_{i}, T \chi_{i}\right)$ nor the velocities of the points, i.e. the direction of the arrows that are associated to the effective temperatures, are constrained. This case represents Eqs. (16) and (29). The full line sketches the parametric plot $\tilde{\chi}(C)$ for the global quantities in a model with two (straight line below $q_{\mathrm{EA}}$ ) and a model with a sequence of global correlation-scales (curve below $q_{\mathrm{EA}}$ ). The dashed line is the continuation of the section where the FDT holds. The black dots indicate the location of the averaged values for the susceptibility and correlation, $\chi_{\mathrm{AV}}=1 / N \sum_{i} \chi_{i}$ and $C_{\mathrm{AV}}=1 / N \sum_{i} C_{i}$ at the times $t$ and $t_{w}$.

\section{Far away times: locked scales}

Naturally, one expects that the fluctuations in the local correlations and integrated linear responses at far away times are not completely independent and that they are constrained to satisfy certain relations. Here we explore the consequences of the scaling expressed in Eq. (22) for all sites in the sample. Hence,

$$
\lim _{t_{w} \rightarrow \infty C\left(t, t_{w}\right)=\mathrm{C}} \chi_{i}\left(t, t_{w}\right)=\tilde{\chi}_{i}\left(\tilde{f}_{i}(\mathrm{C})\right)=\bar{\chi}_{i}(\mathrm{C})
$$

with $\mathrm{C}$ the chosen value of the global correlation. Note that there is no constraint on the values of $\bar{\chi}_{i}(\mathrm{C})$ and $C_{i}=\tilde{f}_{i}(\mathrm{C})$. Thus, the pairs $\left(C_{i}, T \chi_{i}\right)$ can be scattered all over the plane.

However, since all sites are locked to evolve in the same time-scale as the global correlation for each choice of times $\left(t, t_{w}\right)$, one expects

$$
-\beta_{i}^{\mathrm{EFF}}\left(C_{i}\right) \equiv \frac{d \tilde{\chi}_{i}\left(C_{i}\right)}{d C_{i}}=-\beta^{\mathrm{EFF}}(\mathrm{C}),
$$

based on the expectation of having partial equilibration between observables in the same time-scales [59]. Thus, if the scaling in Eq. (22) holds for all sites in the sample, there should be a site independent effective temperature.

The content of Eqs. (20) and (31) is illustrated in Figs. 3 and 4 for a system with two correlation-scales and a system with a continuous sequence of correlation scales, respectively.

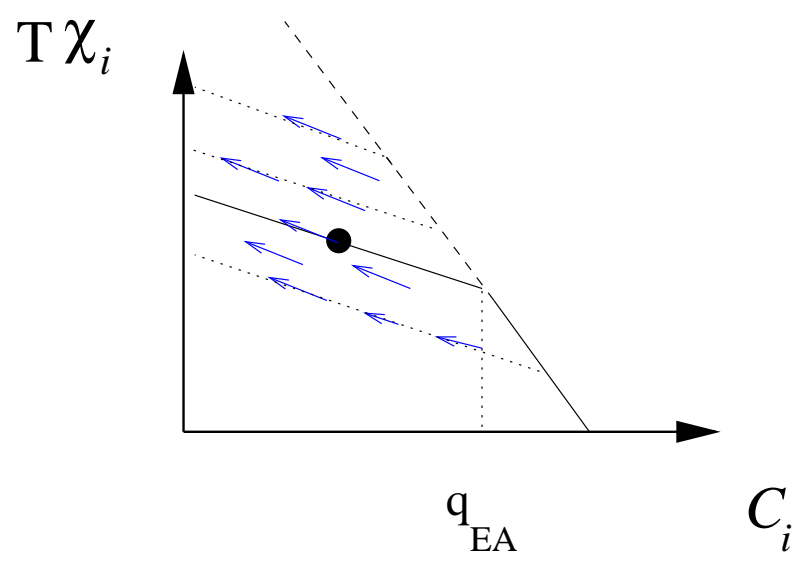

FIG. 3. Fluctuations in a system with only two correlation scales defined using the global correlator. The plot shows fluctuations in the local Edwards-Anderson parameter, $q_{\mathrm{EA}}^{i}$. $\left(t, t_{w}\right)$ are such that the global correlation goes below $q_{\mathrm{EA}}$ and the pairs $\left(C_{i}, T \chi_{i}\right)$ are not constrained to lie close to the global curve, $\tilde{\chi}(C)$, indicated with a full broken line. The effective temperature is the same for all sites, as shown by the fact that all arrows are parallel to the second piece of the global curve. Consequently, once a site enters a "track" after leaving $q_{\text {EA }}^{i}$ (indicated with straight lines drawn with dots), it will follow it as time $t$ evolves. The dashed line is the continuation of the part where the FDT holds. The black dot indicates the location of the values for the susceptibility and correlation averaged over the distribution.

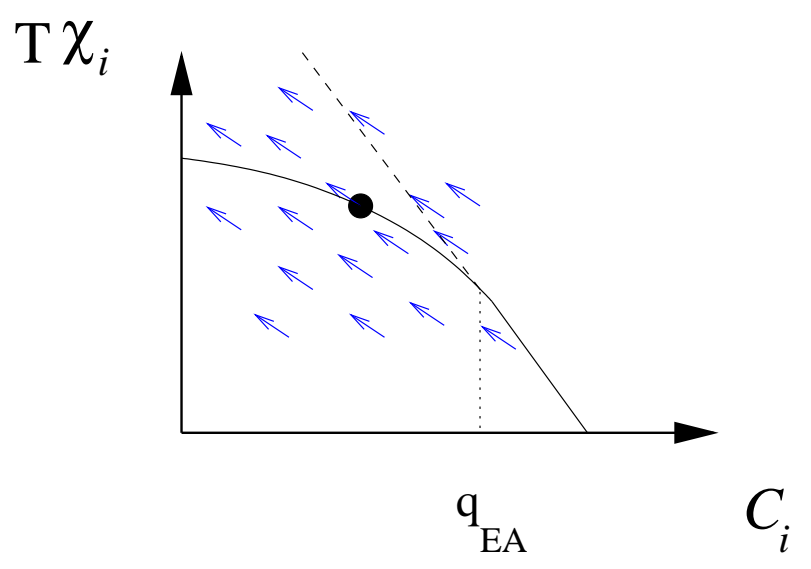

FIG. 4. Fluctuations in a system with a sequence of correlation scales defined using the global correlator. The points are scattered on the plane with no constraint on their positions. The local Edwards-Anderson parameter fluctuates. The velocities with which the points move are all the same. Their positions are not constrained but their velocity moduli and directions are all identical. This is given by the slope at the value of the global correlation at the chosen times $\left(t, t_{w}\right)$, indicated with a black dot on the global line.

Note that in this time regime, at longer times $t$ the 
points will also be scattered on the plane but the average of the correlation will take a smaller value. Since this determines the velocity of the points (and their effective temperature) the direction of the arrows will be different. We can say that there is a nonvanishing acceleration.

One finds two correlation scales in the noise and disorder averaged wavevector-dependent two-time functions of a $d$ dimensional manifold moving in a short-ranged random potential in infinite dimensions [49]. It has also been found in the noise-averaged local dynamics of a dilute ferromagnetic model [38] and in molecular dynamic simulations of Lennard-Jones mixtures $[53,54]$ for different wave-vectors.

\section{Far away times: fluctuations in time-scales}

As opposed to the case just discussed, the scaling in Eq. (18) means that different sites might evolve on different time-scales (if $\left|\delta h_{i}\right| \gg h(t)$ ) and hence have their own effective temperature.

As we shall argue in Sec. III, under certain circumstances the local responses and fluctuations are constrained to follow the global curve, i.e.

$$
\tilde{\chi}_{i}\left(C_{i}\right)=\tilde{\chi}\left(C_{i}\right)
$$

with $C$ and $\tilde{\chi}(C)$ defined in Eqs. (3) and (14), respectively. Then we have

$$
-\beta_{i}^{\mathrm{EFF}}\left(C_{i}\right) \equiv \frac{d \tilde{\chi}_{i}\left(C_{i}\right)}{d C_{i}}=\frac{d \tilde{\chi}\left(C_{i}\right)}{d C_{i}}=\left.\frac{d \tilde{\chi}(C)}{d C}\right|_{C=C_{i}} .
$$

If there are only two time-scales for the decay of the global correlation and, below the global EdwardsAnderson parameter, $\tilde{\chi}(C)$ is linearly dependent on $C$, this equation yields

$$
\beta_{i}^{\mathrm{EFF}}=\beta^{\mathrm{EFF}}
$$

for all sites, and values of $C_{i}<q_{\mathrm{EA}}$.

If, on the contrary, the global correlation decays in a sequence of scales and $\tilde{\chi}(C)$ is not a linear function of $C$, one has fluctuations in the local effective temperature due to the fluctuations in $C_{i}$.

This behavior is sketched in Figs 5 and 6 for a system with two global correlation-scales and a system with a sequence of global correlation-scales, respectively. In [34] we showed that the coarse-grained two-time quantities in the $3 d$ EA model behave as in Fig. 6 . We expect that the coarse-grained two-time correlators of structural glasses will show a behavior as in Fig. 5 .

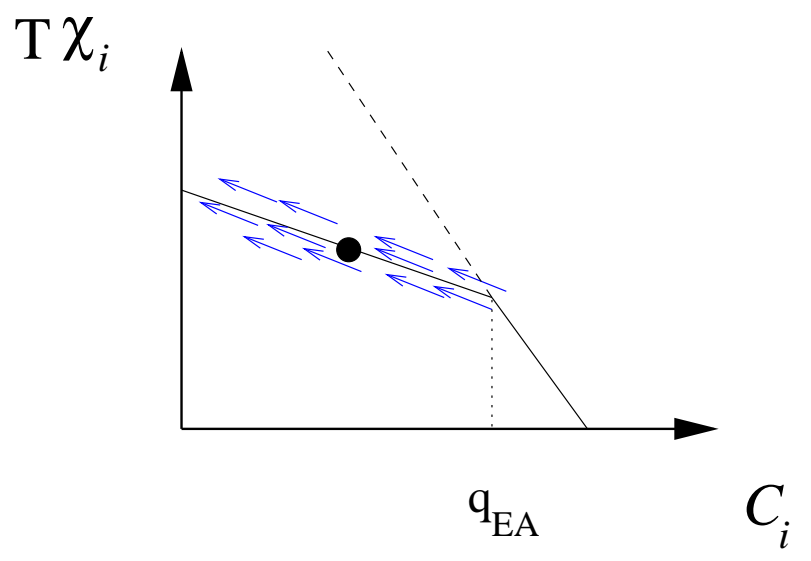

FIG. 5. Fluctuations in a system with a single correlation scale below the Edwards-Anderson plateau. The constraint on the location of the pairs $\left(C_{i}, T \chi_{i}\right)$ implies that there are no fluctuations of the local Edwards-Anderson parameter and that all pairs are concentrated along the global straight line with little dispersion perpendicular to it. The arrows have the direction of the second slope in the global $\tilde{\chi}(C)$ curve due to the result in Eq. (34). As in previous figures, the dashed line continues the FDT line and the black dot indicates the location of the values for the susceptibility and correlation averaged over the distribution.

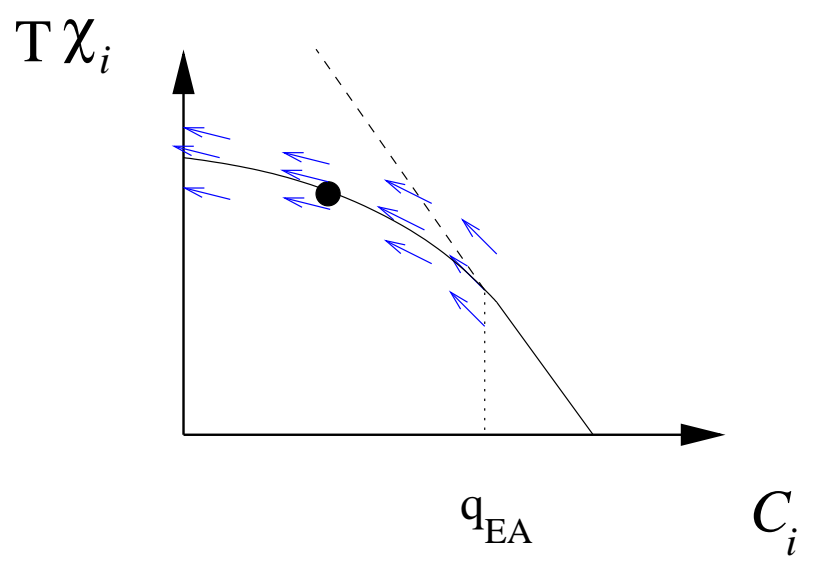

FIG. 6. This is the possibility expressed in Eqs. (32) and (33) for a system with a sequence of correlation scales below the global $q_{\mathrm{EA}}$. All point positions are constrained to be near the global $\tilde{\chi}(C)$ curve for $C<q_{\mathrm{EA}}$ [Eq. (32)], and their velocities are forced to be parallel to the global $\tilde{\chi}(C)$ curve for $C<q_{\mathrm{EA}}$ [Eq. (33)]. The velocities fluctuate from site to site, but since they are determined by the global curve, they are identical for all sites with the same value of the local correlation $C_{i}$. The dashed line and the black dot are as in previous figures.

\section{CONSTRAINTS ON THE LOCAL FLUCTUATIONS}

We recently studied the symmetry properties of the dynamic action for the aging dynamics of finite, $d$ - 
dimensional spin-glasses [33]. Using the SchwingerKeldysh functional formalism we derived a disorderaveraged dynamic generating functional that is a path integral over local two-time functions, $Q_{i}^{\alpha \beta}\left(t, t_{w}\right)$, with $\alpha, \beta=0,1$ labelling the Keldysh components (see details below). This generating functional becomes the classical one, derived in Ref. [61], when $\hbar \rightarrow 0$. This treatment allowed us to discuss the classical and quantum problems simultaneously. The aim of that work was to show that the slow part of the action is a fixed point of a group of time-reparametrizations $(\mathrm{R} p \mathrm{G})$ and to build upon this result an argument to constrain how the local fluctuations should behave in time and space. Here we recall this argument and summarize its main consequences. Indeed, one of the main results arising from this analysis is that the coarse-grained two-time quantities should behave as in Fig. 5 or Fig. 6 if the global correlations decay on one or a sequence of scales, respectively.

\section{A. Time-reparametrization transformations}

The local two-time fields $Q_{i}^{\alpha \beta}\left(t, t_{w}\right)$ are related to the fluctuating local physical two-time functions. For example, the expectation values $\left\langle\left\langle Q_{i}^{\alpha \beta}\left(t, t_{w}\right)\right\rangle\right\rangle$ (we use $\langle\langle\cdots\rangle\rangle$ to denote expectation with respect to the $Q$-action $S[Q])$ give the disorder and thermal averaged local correlations and instantaneous responses:

$$
\begin{aligned}
& \left\langle\left\langle Q_{i}^{00}\left(t, t_{w}\right)\right\rangle\right\rangle=\left\langle\left\langle Q_{i}^{K}\left(t, t_{w}\right)\right\rangle\right\rangle \\
& =\sum_{j} K_{i j}\left[C_{j}^{n a}\left(t, t_{w}\right)\right]=\sum_{j} K_{i j}\left[\left\langle s_{j}(t) s_{j}\left(t_{w}\right)\right\rangle\right], \\
& \left\langle\left\langle Q_{i}^{01}\left(t, t_{w}\right)\right\rangle\right\rangle=\left\langle\left\langle Q_{i}^{R}\left(t, t_{w}\right)\right\rangle\right\rangle \\
& =\sum_{j} K_{i j}\left[R_{j}^{n a}\left(t, t_{w}\right)\right]=\sum_{j} K_{i j}\left[\left.\frac{\left\langle\delta s_{j}(t)\right\rangle}{\delta \eta_{j}\left(t_{w}\right)}\right|_{h=0}\right] .
\end{aligned}
$$

The connectivity matrix $K_{i j}$ contains the variance of the random exchanges $J_{i j}$. For example, for the EdwardsAnderson model with Gaussian distributed disorder, $K_{i j}=J$ for nearest neighbors $i, j$, and zero otherwise. Notice that the $\left\langle\left\langle Q_{i}^{\alpha \beta}\right\rangle\right\rangle$ are already an average over a small region around site $i$ (because of the $K_{i j}$ ) even before coarse graining. There are two other components of the $2 \times 2$ matrix $Q_{i}: Q_{i}^{10}\left(t, t_{w}\right)=Q_{i}^{A}\left(t, t_{w}\right)$ is related to the advanced response and the remaining two-time function $Q_{i}^{11}\left(t, t_{w}\right)=Q_{i}^{D}\left(t, t_{w}\right)$ is related to a correlator whose average $\left\langle\left\langle Q_{i}^{D}\left(t, t_{w}\right)\right\rangle\right\rangle$ vanishes for all causal problems. The two-time fields $Q_{i}^{\alpha \beta}\left(t, t_{w}\right)$ in the action are fluctuating quantities.

We defined a time-reparametrization transformation $t \rightarrow h(t)$ that acts on the fluctuating fields $Q_{i}^{\alpha \beta}\left(t, t_{w}\right)$ as $[33,48]$

$\hat{Q}_{i}^{\alpha \beta}\left(t, t_{w}\right) \equiv\left(\frac{d h(t)}{d t}\right)^{\Delta_{A}^{Q_{i}}}\left(\frac{d h\left(t_{w}\right)}{d t_{w}}\right)^{\Delta_{R}^{Q_{i}}} Q_{i}^{\alpha \beta}\left(h(t), h\left(t_{w}\right)\right)$ where $\Delta_{A}^{Q_{i}}$ and $\Delta_{R}^{Q_{i}}$ are the advanced and retarded scaling dimensions of the field $Q_{i}$ under the rescaling of the time coordinates [48]. These transformations generalize the well-known ones for the expected values of the global $Q$ 's $[5-7,50,61-64]$ to the local and fluctuating case, with $h(t)$ a differentiable function. This choice of transformation is motivated as follows.

Since the local fields $Q_{i}$ are related to the local correlation, linear response and causality breaking correlator, it is natural to associate with them the same scaling dimensions that correspond to the global counterparts of these two-point functions. The appearance of an (approximate) time-reparametrization invariance of the slow part of the relaxation was noticed long ago in the asymptotic solution of the dynamics of mean-field models $[5-7,50,61-64]$. Indeed, when one studies the effective dynamic equations for the slow decay, one drops the timederivatives and approximates the time-integrals. These approximations can be justified because the neglected terms are irrelevant in the $\mathrm{R} p \mathrm{G}$ sense [48]. After applying these approximations the resulting equations become invariant under time-reparametrizations that transform the global correlator and linear response according to

$$
\begin{aligned}
\hat{C}\left(t_{1}, t_{2}\right) & =C\left(h(t), h\left(t_{w}\right)\right), \\
\hat{R}\left(t, t_{w}\right) & =\frac{d h\left(t_{w}\right)}{d t_{w}} R\left(h(t), h\left(t_{w}\right)\right) \theta\left(t-t_{w}\right),
\end{aligned}
$$

for any differentiable and monotonic function $h(t)$ [65]. Note that once one proposes this transformation of the correlator, the transformation of the linear response is forced to take the form in Eq. (39) if one wishes to preserve their link via the FDT.

Extending these definitions to the local and fluctuating fields $Q_{i}^{\alpha \beta}$ we propose:

$$
\begin{gathered}
\left(\Delta_{A}^{Q_{i}^{K}}, \Delta_{R}^{Q_{i}^{K}}\right)=(0,0) \\
\left(\Delta_{A}^{Q_{i}^{R}}, \Delta_{R}^{Q_{i}^{R}}\right)=(0,1), \\
\left(\Delta_{A}^{Q_{i}^{A}}, \Delta_{R}^{Q_{i}^{A}}\right)=(1,0), \\
\left(\Delta_{A}^{Q_{i}^{D}}, \Delta_{R}^{Q_{i}^{D}}\right)=(1,1) .
\end{gathered}
$$

This explains the choice of indices 0,1 for the SchwingerKeldysh components, which conveniently label both the matrix components and scaling dimensions at the same time.

Note that all sites are transformed in the same way under the reparametrization of time just defined. This is a global transformation that leaves invariant any local FDR of the form

$$
\int_{t_{w}}^{t} d t^{\prime} Q_{i}^{01}\left(t, t^{\prime}\right)=f\left(Q_{i}^{00}\left(t, t_{w}\right)\right)
$$


cfr. Eq. (37). This relation is a "constant of motion" with respect to this symmetry. Explicitly:

$$
\begin{aligned}
\int_{t_{w}}^{t} d t^{\prime} \hat{Q}_{i}^{01}\left(t, t^{\prime}\right) & =\int_{t_{w}}^{t} d t^{\prime}\left(\frac{d h\left(t^{\prime}\right)}{d t^{\prime}}\right) Q_{i}^{01}\left(t, t^{\prime}\right) \\
& =\int_{h_{w}}^{h} d h^{\prime} Q_{i}^{01}\left(h, h^{\prime}\right)=f\left(Q_{i}^{00}\left(h, h_{w}\right)\right) \\
& =f\left(\hat{Q}_{i}^{00}\left(t, t_{w}\right)\right) .
\end{aligned}
$$

These transformations imply that the physical noise and disorder averaged saddle-point values transform as expected,

$$
\begin{aligned}
{\left[C_{i}^{n a}\left(t, t_{w}\right)\right] } & \rightarrow\left[C_{i}^{n a}\left(h(t), h\left(t_{w}\right)\right)\right], \\
{\left[\chi_{i}^{n a}\left(t, t_{w}\right)\right] } & \rightarrow\left[\chi_{i}^{n a}\left(h(t), h\left(t_{w}\right)\right)\right],
\end{aligned}
$$

and the FDR in Eq. (26) is respected.

\section{B. Invariance of the action}

In Ref. [33] we studied the symmetries of the action $S[Q]$. We showed that the long-time limit of the effective Landau-Ginzburg aging action for the two-point functions $Q_{i}^{\alpha \beta}\left(t, t_{w}\right)$ is a fixed point of the group of time reparametrizations $(\mathrm{R} p \mathrm{G})$. By this we mean that, after separating the field $Q_{i}^{\alpha \beta}\left(t, t_{w}\right)=Q_{i \text { FAST }}^{\alpha \beta}\left(t, t_{w}\right)+$ $Q_{i \text { SLOW }}^{\alpha \beta}\left(t, t_{w}\right)$, and then integrating out the fast part of the fields, all terms in the effective slow action are invariant under a reparametrization of time, $t \rightarrow h(t)$, that transforms the fields as in Eq. (37). In deriving the effective action for the slow contribution $Q_{i}^{\alpha \beta}$ sLow $\left(t, t_{w}\right)$, we assumed that there is a local separation of time scales. The only other ingredient in the proof was that the system must be causal.

The approach to the fixed point is asymptotic, and there will be corrections to scaling at finite times. In particular, the kinetic contribution to the effective action is irrelevant at long times. However, irrelevant as it is at long times, this term acts as a (time-decaying) symmetry breaking field that selects a particular reparametrization.

The importance of what we have shown is that it holds for infinite and short-range models alike, and at the level of the action, not just the equations of motion. Moreover, it suggests an approach to study spatial fluctuations of aging dynamics, as we discuss below.

\section{Implications of $\mathbf{R} p \mathbf{G}$ invariance - connection with a sigma model}

In view of this approximate (asymptotic) symmetry we constructed an argument that allowed us to predict how the local fluctuations of the disordered averaged theory should behave. In this section we explain in more detail the arguments sketched in $[33,34]$.

\section{Parallel with the $O(N)$ model}

To better explain the argument, it is useful to explore an analogy with the static, coarse-grained, $O(N)$ theory of magnets in $d$ dimensions:

$$
\mathcal{H}=\int d^{d} r\left[(\vec{\nabla} \cdot \vec{m}(\vec{r}))^{2}+\vec{H} \cdot \vec{m}(\vec{r})+V(\vec{m})\right],
$$

where $\vec{m}(\vec{r})$ is a continuous $N$-dimensional variable, $\vec{m}(\vec{r})=\left(m^{1}(\vec{r}), m^{2}(\vec{r}), \ldots, m^{N}(\vec{r})\right)$, that represents the local magnetization. $V(\vec{m})$ is a potential energy with the form of a Mexican hat. $\vec{H}$ is an external magnetic field. A particular case of this model is the well-known $3 d$ Heisenberg ferromagnet obtained when $N=3$ and $d=3$.

The parallel between the two models is as follows.

1. Fields - The two-time fields $Q_{i}^{\alpha \beta}\left(t, t_{w}\right)$, once coarsegrained over a volume $V$, play the role of the static magnetization $\vec{m}(\vec{r})$.

2. Symmetry - When $\vec{H}=0$ the energy function of the magnetic problem is invariant under a global rotation of the magnetization, $m^{a}(\vec{r})=R^{a b} m^{b}(\vec{r})\left(R^{a b}(\vec{r})=R^{a b}\right.$, for all $\vec{r})$. The potential $V(\vec{m})$ has a zero mode along the bottom of the Mexican hat potential.

In the dynamic problem, for longer and longer waitingtimes, the symmetry breaking terms become less and less important and the action progressively acquires a global symmetry (a zero mode develops).

3. Spontaneous symmetry breaking - In the absence of a pinning field, the ferromagnetic model spontaneously chooses a direction of the vector $\vec{m}$ everywhere in real space, $\vec{m}(\vec{r})=\vec{m}_{0}$ in the broken symmetry phase.

In the $\mathrm{R} p \mathrm{G}$ invariant asymptotic regime, the minima of the dynamic action satisfy the global reparametrization symmetry. A given direction in the minima manifold is described by one uniform time-reparametrization everywhere in space, $h(\vec{r}, t)=h(t)$,

4. Explicit symmetry breaking - A non-zero magnetic field $\vec{H}$ breaks the symmetry explicitly by tilting the Mexican hat potential. It forces the magnetization, $\vec{m}$, to point in its direction in the $N$ dimensional space everywhere in the real $d$-dimensional space.

$\mathrm{R} p \mathrm{G}$ irrelevant terms, which vanish asymptotically, play the role of (time-decaying) symmetry breaking fields that select a particular time-reparametrization. The particular scaling function $h(t)$ chosen by the system is determined by matching the fast and the slow dynamics. It depends on several details - the existence of external forcing, the nature of the microscopic interactions, etc. In other words, the fast modes which are absent in the slow dynamics act as symmetry breaking fields for the slow modes.

5. Fluctuations - These correspond to smooth variations in the magnetization as a function of position, 
$\vec{m}(\vec{r})=\vec{m}_{0}+\delta \vec{m}(\vec{r})$. There are two types of fluctuations, longitudinal and transverse. The former change the length of the magnetization vector, $|\vec{m}(\vec{r})|$; these move off the saddle point manifold of potential minima, and are thus massive excitations. The latter change direction only, $\vec{m}_{0} \cdot \delta \vec{m}(\vec{r})=0$; these remain in the potential minima manifold, and are massless excitations. The transverse fluctuations, which correspond to smoothly spatially varying rotations of the magnetization vector, are therefore the most energetically favored. These are the spin-waves or Goldstone modes in the $O(N)$ model.

The equivalent of the transverse modes for the dynamic $Q$-theory are smooth spatially-varying time reparametrizations, $h(\vec{r}, t)=h(t)+\delta h(\vec{r}, t)$. Uniform or global reparametrization is the symmetry of the model; the smooth spatially fluctuating $\delta h(\vec{r}, t)$ are excitations that cost the lowest action, or the Goldstone modes of the $Q$-action.

What controls the distance scale on which the fluctuations can vary? The first term in the ferromagnetic model in Eq. (48) restricts the magnitude of the variation of the direction of $\vec{m}$. For the second model, one expects that a similar stiffness will be generated once it is coarsegrained, making sharp variations in $\delta h(\vec{r}, t)$ difficult to achieve.

The longitudinal fluctuations in the ferromagnet, those in which the modulus of the magnetization vector changes, $m=m_{0}+\delta m$, cost more energy due to the Mexican hat potential and hence are less favorable. Similarly, in the dynamic problem the fluctuations in which the "external form" [for example, imagine the function $f$ in Eq. (18) varying with position] of the coarse-grained two-time functions changes are less favorable.

\section{Spatial fluctuations of aging dynamics}

The penalties for longitudinal fluctuations, and the soft transverse fluctuations corresponding to local reparametrizations $t \rightarrow h(\vec{r}, t)$, led us to propose that the coarse-grained local correlations in the aging regime scale as in Eq. (17). In the $C-T \chi$ plane, these soft modes correspond to displacements along the global $\tilde{\chi}(C)$ curve; this is shown in Fig. 7. Displacements that move points off the $\tilde{\chi}(C)$ curve are the longitudinal modes, which do not correspond to smoothly varying time reparametrizations.

A natural consequence of this is the prediction that the fluctuations in the coarse-grained local FDR should be such that the distribution of points $\chi_{i}^{c g}\left(C_{i}^{c g}\right)$ follow the global FDR, $\tilde{\chi}(C)$, defined in Eq. (14). Thus, Eq. (33) should hold (see Fig. 6). Given any pair of times $\left(t, t_{w}\right)$ such that the global correlation equals a prescribed value, $C\left(t, t_{w}\right)=\mathrm{C}$, the local effective temperature should be the same for all regions of space having the same value of the local correlation $C_{i}\left(t, t_{w}\right)$.

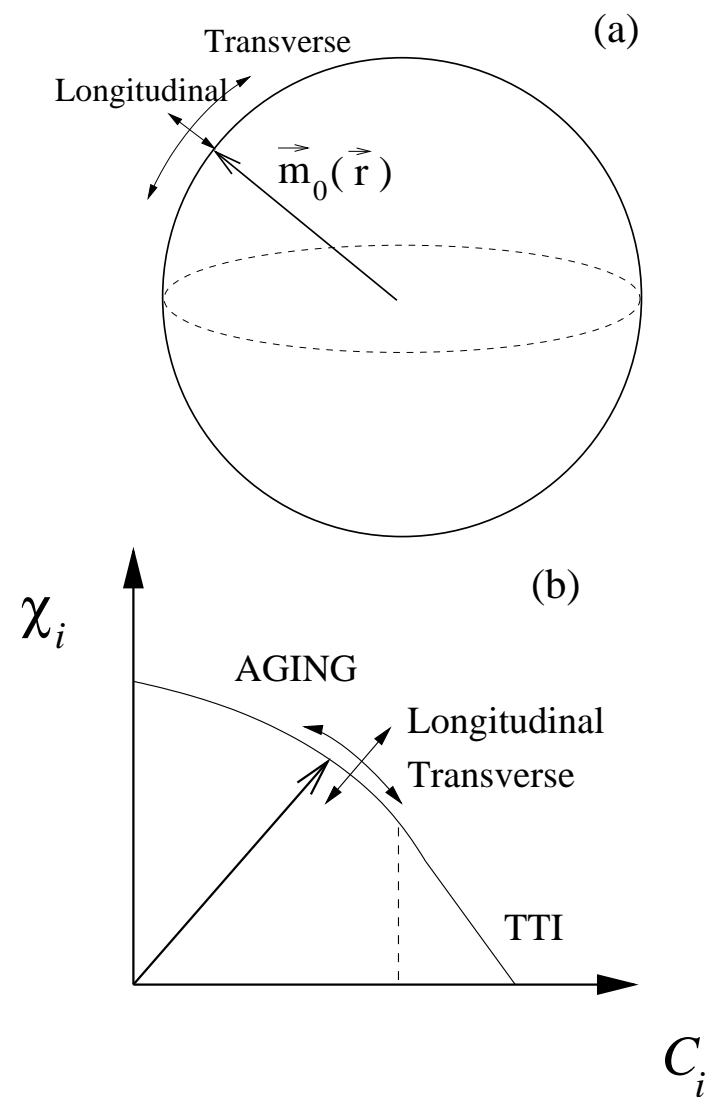

FIG. 7. Panel a): soft modes in the Heisenberg magnet. $N$-dimensional sphere with radius $\left|\vec{m}_{o}(\vec{r})\right|$ centered at the spatial point $\vec{r}$. The longitudinal and transverse directions for the fluctuations are indicated with arrows. Panel b): soft modes in the spatial fluctuations during aging. The full line represents the global $\tilde{\chi}(C)$ curve in a model with a sequence of correlation scales below $q_{\mathrm{EA}}$. The vertical dashed line shows the value of $q_{\mathrm{EA}}$ separating the slow stationary decay (ТTI) from the slow aging regime. The directions for longitudinal and transverse fluctuations as explained in the text are indicated with arrows.

We would like to remark that the above theoretical argument was developed for the fluctuating fields $Q_{i}^{\alpha \beta}\left(t, t_{w}\right)$, not their thermal averages; these correspond, instead, to the expectation values $\left\langle\left\langle Q_{i}^{\alpha \beta}\left(t, t_{w}\right)\right\rangle\right\rangle$. The average over the disorder is carried from the outset in the field-theoretical approach, while fluctuations due to noise histories are kept by writing the $Q$-theory using a path integral formulation. How can one test the predictions from this disordered average field theory against numerical simulations? The answer lies on whether the distributions that one studies are self-averaging or not. What we find is that, if we study coarse-grained local correlations and integrated responses [34] through numerical simulations, their distributions become independent of the disorder realization for large enough coarse-graining cell sizes. This is an indication that self-averaging holds for these non-equilibrium distributions, and in fact the 
coarse-graining procedure is somehow averaging over disorder. This is perhaps another reason to work with coarse-grained quantities, as opposed to noise averaged quantities.

What can we say about the behavior of noise-averaged (and neither disorder averaged nor coarse-grained) local two-time functions? We argue that this procedure corresponds to "fingerprinting" the particular disorder realization of the system. By a "fingerprint" of disorder we mean that noise-averaged correlations and integrated responses at the different sites purely reflect their disorder environment, since there is no other source of fluctuations left in the problem. Of course, further averaging over the disorder would erase the "fingerprint", and simply give the global result. For this reason, coarse-graining also erases the "fingerprint" for a self-averaging system. The noise-averaging approach should correspond to the scaling in Eq. (17), and fluctuations are then not constrained to follow the global $\tilde{\chi}(C)$ curve, as in Fig. 6 .

\section{Random surface action - effective action for the fluctuations}

In the spirit of the usual approach in deriving a coarsegrained effective action for the relevant fields in a problem [45], we search for the "minimal" effective action describing the aging dynamics of the system in terms of coarse-grained fields $Q^{\alpha \beta}\left(\vec{r} ; t, t_{w}\right)$. More precisely, in Sec. III A we mentioned that the dynamic generating function is expressed in terms of local two-time fields, $Q_{i}^{\alpha \beta}\left(t, t_{w}\right)$, that are coarse-grained over a cube involving the first neighbors of the site $i$. Since we are interested in postulating an effective action for the soft fluctuations we shall consider a further spatial coarse-graining of these fields and work with $Q^{\alpha \beta}\left(\vec{r}, t, t_{w}\right)$ that represents a smooth two-time field at the position $\vec{r}$ in real space.

In a coarse grained theory, one expects gradient terms to be present in the effective action. Their effect is to penalize rapid variations (in time and space) of the coarse grained fields. These terms play the role of the term $\int d^{d} r(\vec{\nabla} \cdot \vec{m}(\vec{r}))^{2}$ in the energy Eq. (48) for the Heisenberg ferromagnet.

As we discussed above, global $\mathrm{R} p \mathrm{G}$ invariance is, in the limit of long times, a symmetry of the total action and we should preserve it when constructing the effective action for the fields $Q_{i}^{\alpha \beta}\left(\vec{r} ; t, t_{w}\right)$. Thus, this symmetry poses strong restrictions on the form of allowed gradient terms.

In general, the effective action takes the form:

$$
S[Q]=S_{\text {grad }}[Q]+S_{\text {local }}[Q],
$$

with $S_{\text {local }}[Q]$ an $\mathrm{R} p \mathrm{G}$ invariant action comprised solely of local terms and $S_{\text {grad }}[Q]$ including the gradient (nonlocal) dependence. Let us propose, for the latter,

$$
\begin{aligned}
S_{\text {grad }}[Q]=- & \int d^{d} r \int_{0}^{\infty} d t_{1} \int_{0}^{\infty} d t_{2}\left(\nabla \partial_{t_{1}} Q^{00}\left(\vec{r} ; t_{1}, t_{2}\right)\right) \\
& \times\left(\nabla \partial_{t_{2}} Q^{00}\left(\vec{r} ; t_{1}, t_{2}\right)\right)
\end{aligned}
$$

Notice that a global $\mathrm{R} p \mathrm{G}$ transformation $t \rightarrow h(t)$, $Q^{00}\left(\vec{r} ; t_{1}, t_{2}\right) \rightarrow \hat{Q}^{00}\left(\vec{r} ; t_{1}, t_{2}\right)=Q^{00}\left(\vec{r} ; h\left(t_{1}\right), h\left(t_{2}\right)\right)$ leaves $S_{\text {grad }}[Q]$ invariant as well, as can be explicitly checked:

$$
\begin{aligned}
S_{\text {grad }}[\hat{Q}]=- & \int d^{d} r \int_{0}^{\infty} d t_{1} \int_{0}^{\infty} d t_{2}\left(\nabla \partial_{t_{1}} \hat{Q}^{00}\left(\vec{r} ; t_{1}, t_{2}\right)\right) \\
& \times\left(\nabla \partial_{t_{2}} \hat{Q}^{00}\left(\vec{r} ; t_{1}, t_{2}\right)\right) \\
=- & \int d^{d} r \int_{0}^{\infty} d h_{1} \int_{0}^{\infty} d h_{2}\left(\nabla \partial_{h_{1}} Q^{00}\left(\vec{r} ; h_{1}, h_{2}\right)\right) \\
& \times\left(\nabla \partial_{h_{2}} Q^{00}\left(\vec{r} ; h_{1}, h_{2}\right)\right)=S_{\text {grad }}[Q] .
\end{aligned}
$$

Before proceeding, we would like to remind the reader that $Q^{00}$ and $C^{c g}$ are indeed related, so what we discuss below applies to the spatially-varying coarsegrained correlation $C^{c g}\left(\vec{r} ; t_{1}, t_{2}\right)$. The expectation value $\left\langle\left\langle Q^{00}\left(\vec{r} ; t_{1}, t_{2}\right)\right\rangle\right\rangle$ is related to the noise (and disorder) average of $C^{c g}\left(\vec{r} ; t_{1}, t_{2}\right)$ [see Eq. (35)]. Similarly, one can show a relation between all $n$-moments $\left\langle\left\langle Q^{00}\left(\vec{r}_{1} ; t_{1}, t_{2}\right) \cdots Q^{00}\left(\vec{r}_{n} ; t_{1}, t_{2}\right)\right\rangle\right\rangle$ and the noise (and disorder) average of $C$ 's at $n$ points. Hence, the fluctuations of these quantities are akin.

The reader might also note that there are several other simple $\mathrm{R} p \mathrm{G}$ invariant actions that we could have written down, involving either $Q^{01}$ and $Q^{10}$, or $Q^{11}$ and $Q^{00}$. However, such terms vanish when evaluated on a saddlepoint configuration that satisfies causality. Since we focus on the causal solution and its fluctuations, we do not consider such terms. The gradient penalizing term in Eq. (50) is chosen as one of the simplest non-trivial terms that respects $\mathrm{R} p \mathrm{G}$ invariance.

In analogy with the study of the $O(N)$ model and the derivation of an effective action describing the spin waves, we start by identifying the uniform (in space) saddle point configuration and we then consider small fluctuations around it.

Since the saddle-point solution does not depend on the spatial position, $S_{\text {grad }}\left[Q_{\mathrm{SP}}\right]=0$. Thus, $Q_{\mathrm{SP}}$ is completely determined by $S_{\text {local }}[Q]$ and its precise form depends on the details of the model. We argue that one can still learn a great deal about the spatial fluctuations by considering simplified, approximate saddle-point solutions. We know from numerical simulations of the $3 d$ EA model $[51,23]$ that the global correlation scales rather well as

$$
C\left(t_{1}, t_{2}\right) \approx q_{\mathrm{EA}} f\left(\frac{\min \left(h\left(t_{1}\right), h\left(t_{2}\right)\right)}{\max \left(h\left(t_{1}\right), h\left(t_{2}\right)\right)}\right),
$$

with $h(t)$ and $f(x)$ two monotonic functions. $f(x)$ satisfies $f(1)=1$ and $f(0)=0$. A very good scaling of the data is obtained using $h(t)=\exp \left(t^{1-\mu} /(1-\mu)\right)$ with $\mu$ taking values that are slightly above 1 . The simpler 
scaling $h(t)=t$ is not perfect but it provides a rather good approximation. It will be convenient to define a new time-dependent function $\phi$ by

$$
h(t)=e^{\phi(t)} .
$$

The role of spatially varying rotations in the $O(N)$ model is here played by the spatially varying time reparametrizations that we write as

$$
h(t) \rightarrow h(\vec{r}, t)=e^{\phi(\vec{r}, t)} .
$$

Thus, we express the saddle-point solution as

$$
Q_{\mathrm{SP}}^{00}\left(t_{1}, t_{2}\right) \approx q_{\mathrm{EA}} f\left(e^{-\left|\phi\left(t_{1}\right)-\phi\left(t_{2}\right)\right|}\right),
$$

and we parametrize the fluctuations around it with

$$
Q^{00}\left(\vec{r} ; t_{1}, t_{2}\right) \approx q_{\mathrm{EA}} f\left(e^{-\left|\phi\left(\vec{r}, t_{1}\right)-\phi\left(\vec{r}, t_{2}\right)\right|}\right) .
$$

Let us now split $\phi(\vec{r}, t)=\tau[h(t)]+\delta \phi(\vec{r}, t)$, where $\tau[h(t)]$ selects the global reparametrization, i.e. fixes the "direction" of the saddle-point solution, and $\delta \phi(\vec{r}, t)$ controls the small fluctuations around it. Furthermore, it is best to think of $\tau$ as the proper time variable and work with $\varphi(\vec{r}, \tau) \equiv \delta \phi(\vec{r}, t(\tau))$. Changing integration variables in $S_{\text {grad }}[Q]$ from $t$ to $\tau$, and expanding around small $\varphi$, we obtain [for the simplest case $f(x)=x$ ]:

$$
\begin{aligned}
S_{\mathrm{grad}} \approx & q_{\mathrm{EA}}^{2} \int d^{d} r \int_{-\infty}^{\infty} d \tau_{1} \int_{-\infty}^{\infty} d \tau_{2}\left(\nabla \dot{\varphi}\left(\vec{r}, \tau_{1}\right)\right) \\
& \times\left(\nabla \dot{\varphi}\left(\vec{r}, \tau_{2}\right)\right)\left[1-\left|\tau_{1}-\tau_{2}\right|\right]^{2} e^{-2\left|\tau_{1}-\tau_{2}\right|} \\
= & \frac{1}{2} q_{\mathrm{EA}}^{2} \int d^{d} r \int_{-\infty}^{\infty} d \tau(\nabla \dot{\varphi}(\vec{r}, \tau))^{2}+\cdots,
\end{aligned}
$$

where $\dot{\varphi}=\partial \varphi / \partial \tau$. In Eq. (57) we retained only the leading relevant term (we neglected terms with higher $\tau$ derivatives). It is very simple to show that in the case of a general function $f(x)$ the resulting action is identical to the above up to a renormalized stiffness constant, namely:

$$
S_{\text {grad }} \approx \frac{1}{2} \lambda_{f} q_{\mathrm{EA}}^{2} \int d^{d} r \int_{-\infty}^{\infty} d \tau(\nabla \dot{\varphi}(\vec{r}, \tau))^{2}+\cdots,
$$

with

$$
\lambda_{f}=4 \int_{0}^{1} d x x\left\{[1+\ln x] f^{\prime}(x)+x \ln x f^{\prime \prime}(x)\right\}^{2}
$$

a positive definite constant. In particular, for a power law $f(x)=x^{\lambda}$, the renormalization factor of the stiffness is simply $\lambda_{f}=\lambda$.

The $\varphi(\vec{r}, \tau)$ that parametrize the fluctuations can be thought of as the height of a random surface. Equation (56) relates the fluctuations of the local correlators with the reparametrization field $\phi$. In the case $f(x)=x^{\lambda}$, this relation takes the simple form

$$
Q^{00}\left(\vec{r} ; t_{1}, t_{2}\right)=Q_{\mathrm{SP}}^{00}\left(t_{1}, t_{2}\right) e^{-\lambda\left|\varphi\left(\vec{r}, \tau\left(t_{1}\right)\right)-\varphi\left(\vec{r}, \tau\left(t_{2}\right)\right)\right|},
$$

so spatial fluctuations of the correlations $C^{c g}\left(\vec{r} ; t_{1}, t_{2}\right)$ are related to the fluctuations of the height differences from two $\varphi$ surfaces evaluated at two proper times $\tau_{1}$ and $\tau_{2}$, as sketched in Fig. 8. It is simple to understand how these height differences, which fluctuate as a function of time, can very simply explain the sorpassi we discussed in Sec. II E 2.

What are the statistics of these height differences? Because $\dot{\varphi}$ is a Gaussian surface, it is simple to show that

$$
\left\langle\left[\varphi\left(\vec{r}, \tau_{1}\right)-\varphi\left(\vec{r}, \tau_{2}\right)\right]^{2}\right\rangle=2 G_{d}(\vec{r}, \vec{r})\left|\tau_{1}-\tau_{2}\right|,
$$

where $G_{d}\left(\vec{r}, \vec{r}^{\prime}\right)$ is the correlation function for a Gaussian random surface in $d$-dimensions. When $\tau_{1}$ and $\tau_{2}$ get close, irrelevant terms neglected in Eq. (57) become important, and $\left|\tau_{1}-\tau_{2}\right|$ should be replaced by a short-time cut-off $\tau_{c}$. The details of the crossover depend on those neglected terms, but one can capture its most important features by approximately replacing $\left|\tau_{1}-\tau_{2}\right| \rightarrow \tau_{c}+\left|\tau_{1}-\tau_{2}\right|$ in Eq. (61).

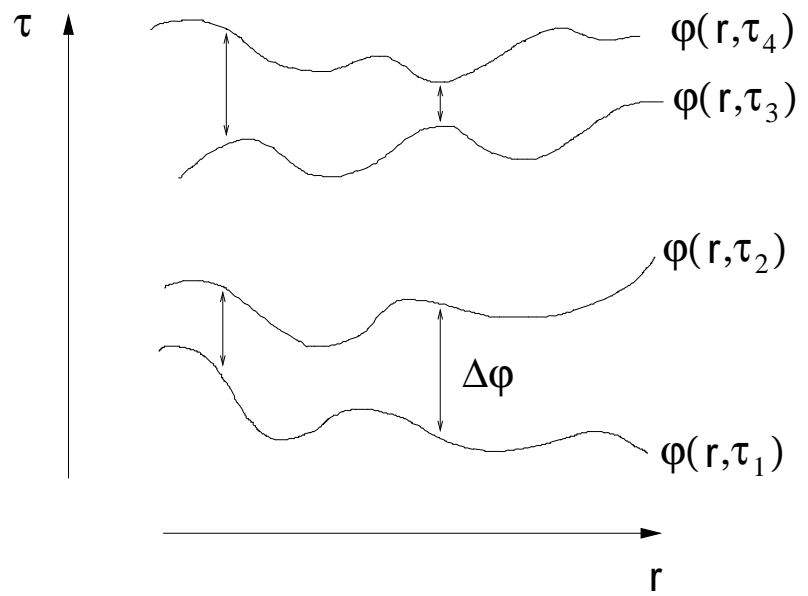

FIG. 8. Schematics of the height separation between surfaces $\varphi(\vec{r}, \tau)$ at different proper times $\tau_{1}, \cdots, \tau_{4}$. Notice that height differences for two different points $\vec{r}, \vec{r}^{\prime}$ can increase or decrease for different pairs of $\tau$ 's. Such fluctuations can explain the sorpassi we described previously.

The simple expression Eq. (61) has some powerful consequences. First, it implies that the statistical distribution of local correlation functions $C\left(\vec{r} ; t_{1}, t_{2}\right)$ is controlled by the proper time difference $\tau_{1}-\tau_{2}$. Because $\tau=\ln h(t)$ follows from the scaling of the global correlations of the EA model $[51,23]$, we can conclude that the full PDF for the local correlations is also a function that scales with the ratio $h(t) / h\left(t_{w}\right)$; this scaling is confirmed in our numerical simulations in the $3 d$ EA model, as discussed in Sec. IV D, where for simplicity, we use $h(t) \approx t$. 
Second, it suggests that fluctuations of the height differences are much more pronounced in $2 d$ than in $3 d$. $G_{d}(\vec{r}, \vec{r})$, the correlation function for a Gaussian random surface in $d$-dimensions, goes to an ultraviolet cut-off dependent constant in $3 d$, but diverges logarithmically with the system size $L$ in $2 d$. Hence, this may provide a simple explanation why in $2 d$ fluctuations destroy the global order in spite of the existence of non-zero local correlations.

Additionally, Eq. (61) allows us to obtain more detailed predictions about the leading order behavior of the fluctuating reparametrization. Within the present approximation, $\varphi\left(\vec{r}, \tau_{1}\right)-\varphi\left(\vec{r}, \tau_{2}\right)$ is a Gaussian random variable with a variance given by the square root of the r.h.s. of Eq. (61). Combining this with the physical information that $\tau(t)=\ln h(t)$ (where again for simplicity we can take $h(t) \approx t$ for the scaling of the global functions), and noting $\phi(\vec{r}, t)=\tau(t)+\delta \phi(\vec{r}, t)$, we can write the following scaling form:

$$
\begin{aligned}
\phi\left(\vec{r}, t_{1}\right)-\phi\left(\vec{r}, t_{2}\right)=\ln t_{1}-\ln t_{2} \\
+\left[G_{d}(\vec{r}, \vec{r})\left(\tau_{c}+\left|\ln t_{1}-\ln t_{2}\right|\right)\right]^{1 / 2} X_{r}\left(t_{1}, t_{2}\right) \\
=\ln \left(t_{1} / t_{2}\right)+\left[a+b\left|\ln \left(t_{1} / t_{2}\right)\right|\right]^{\alpha} X_{r}\left(t_{1}, t_{2}\right)
\end{aligned}
$$

where, to Gaussian level $\alpha=1 / 2$, and $X_{r}\left(t_{1}, t_{2}\right)$ is a random Gaussian variable of unit variance and spatially correlated. Higher order corrections could in principle modify this prediction significantly. It turns out that simulational results [34] are consistent with Eq. (62), although with $\alpha \neq 1 / 2$ and with a non-Gaussian distribution for $X_{r}\left(t_{1}, t_{2}\right)$.

\section{SCALING OF LOCAL CORRELATIONS}

After having introduced the local two-time quantities in Sec. II and having discussed several scenarios for their behavior in Sec. III, in this section we study the dynamic behavior of the coarse-grained and noise-averaged local correlations of the $3 d$ EA model using numerical simulations.

\section{A. Global correlation}

We recall that, for the waiting and total times we use, the aging decay of the global correlation [Eq. (3)] is rather well described with a simple $t / t_{w}$ scaling [51]. Indeed, even without subtracting the first approach to the plateau at $q_{\mathrm{EA}}$ (that can occur with a very slow power law [21]) the scaling is quite acceptable. To illustrate this point, we show the global correlation against $\left(t-t_{w}\right) / t_{w}$ in Fig. 9 for a system evolved with a single thermal history and using relatively short waiting-times.

We stress that we do not claim that this scaling holds asymptotically. It simply approximately describes the data for these short waiting-times and up to the maximum waiting-time reachable in simulations, which is of the order of $10^{6}$ Monte Carlo steps (MCs). A better description of the numerical data is obtained if one uses $C\left(t, t_{w}\right) \approx f\left(h(t) / h\left(t_{w}\right)\right)$ with $h(t)=\exp \left(t^{1-\mu} /(1-\mu)\right)$ and $\mu$ slightly larger than 1 (see Refs. [51,23] for a precise analysis where longer waiting-times have been used and the approach to the plateau has been taken into account). In what follows, to keep the analysis of the data as simple as possible, we adopt the simpler power law expression for $h(t)$.

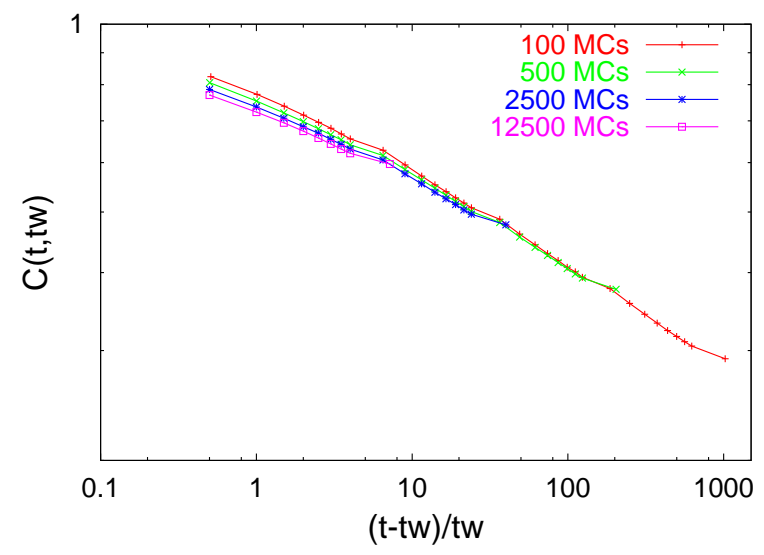

FIG. 9. Decay of the global correlation $C\left(t, t_{w}\right)$ in the $3 d$ EA model as a function of $\left(t-t_{w}\right) / t_{w}$. The waiting-times are indicated in the key. $T=0.7$ and $L=32$.

\section{B. Relation to random surfaces}

The evaluation of the local correlations on the $d$ dimensional real-space substrate allowed us to draw a parallel between the evolution of the local coarse-grained correlations and the dynamics of a $d$-dimensional random surface [34], see Sec. IIID. At any pair of times $\left(t, t_{w}\right)$ the random surface fluctuates about the global value $C\left(t, t_{w}\right)$ and it is constrained to do so between -1 and 1 since $C_{i} \in[-1,1]$. Within our $\mathrm{R} p \mathrm{G}$ invariant theory, there is a one-to-one relationship between this random surface for the coarse-grained correlator $C^{c g}\left(\vec{r} ; t, t_{w}\right)$ and the random surfaces $\phi(\vec{r}, t)$ (and $\phi\left(\vec{r}, t_{w}\right)$ ) discussed in Sec. IIID, as evident from the relation Eq. (56). The parallel between the evolution of the local correlations and the dynamics of a $d$-dimensional random surface can also be extended to the noise-averaged correlations (although we present no analytical theory for this case).

The statistical and dynamical properties of the surfaces in each case are not necessarily the same. In Figs. 10 and 11 we show the values of the local coarse-grained and noise-averaged correlations, respectively, on a $2 d$ cut of the $3 d$ real space. These figures illustrate how the local correlations generate a surface with height $C_{i}$ on each 
site $i$ of the $3 d$ substrate. The statistical properties of the $C_{i}$ (their distribution, geometric organization, etc.) inform us about the statistical properties of the random surface. Similarly, we can think about the random surface generated by the coarse-grained and noise-averaged local susceptibilities. In Sec. IX we shall show the results of the numerical analysis of the geometric properties of the random surface of local correlations in the $2 d$ and $3 d$ EA models.

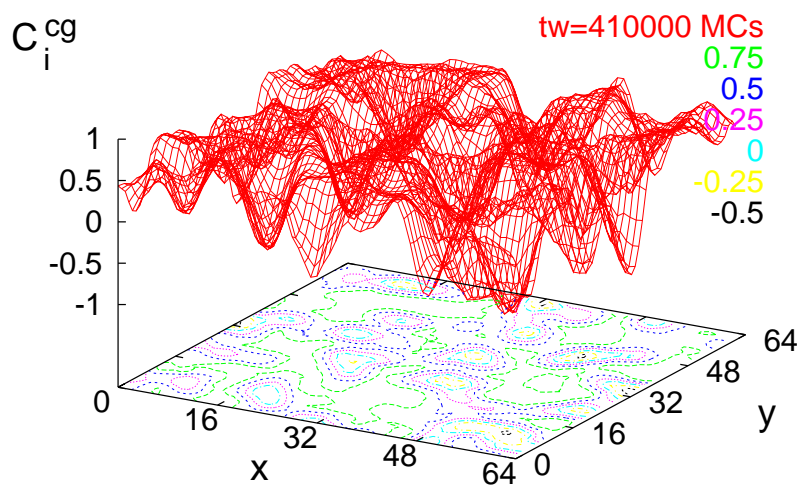

FIG. 10. Surface of local coarse-grained correlations on a $2 d$ cut of the $3 d$ real space. $T=0.8, L=64, M=3$. The local correlations are evaluated at $t_{w}=4.1 \times 10^{5}$ and $t=2.8 \times 10^{6}$, i.e. $t / t_{w} \sim 6.8$.

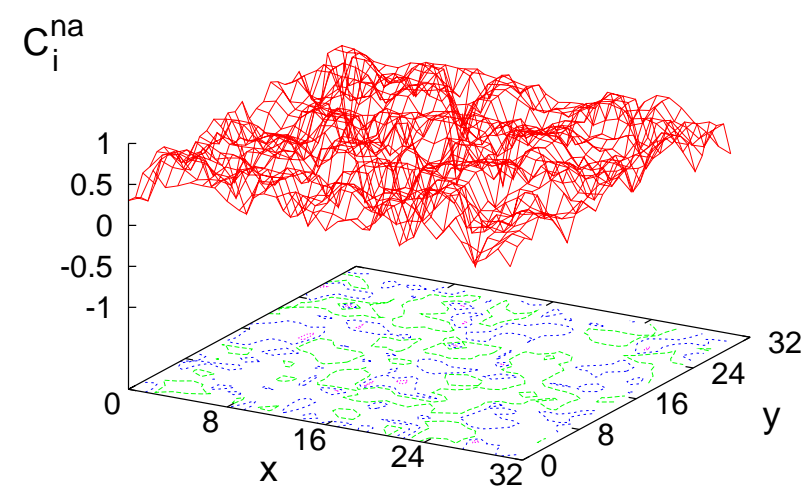

FIG. 11. Surface of local noise-averaged correlations on a $2 d$ cut of the $3 d$ real space. $T=0.7, L=32,10^{3}$ noises. The local correlations are evaluated at $t_{w}=1.6 \times 10^{4}$ and $t=4.8 \times 10^{4}$, i.e. $t / t_{w}=3$. The fluctuations are reduced with respect to the ones in Fig. 10 since $T$ is lower and $t / t_{w}$ is smaller here. Note that this surface fluctuates between 0 and 1 since the noise-averaging eliminates negative values.

\section{Coarse-graining volume}

Before analyzing the time dependence of the local correlations we briefly exhibit the dependence of the coarse-grained correlations on the coarse-graining volume. In Fig. 12 we plot $C_{i}^{c g}\left(t, t_{w}\right)$ against the timedifference $t-t_{w}$ on seven sites around the site with coordinates $(1,1,1)$, using three coarse-graining volumes $V=(2 M+1)^{3}$ with $M=3,6,9$. The waiting-time is $t_{w}=500$ MCs. As expected, coarse-graining smoothens the spatial variation of the local correlations and we see very little variation between the local correlations on neighboring sites. For $t_{w}=500 \mathrm{MCs}$, the curves for $M=3$ are rather noisy while those for $M=6$ and $M=9$ behave roughly in the same way. For longer waiting-times the curves for the three coarse-graining volumes behave roughly in the same manner (not shown). In what follows we shall typically use $M=1, M=3$ and $M=6$ that correspond to linear sizes $2 M+1=3,2 M+1=7$ and $2 M+1=13$. The first choice is the coarse-graining implicit in the analytic theory (see Eqs. (35) and (36), except that here the central site is also included). The two latter choices are of the order of one fifth of the linear size of the system if $L=32$ and $L=64$, respectively.

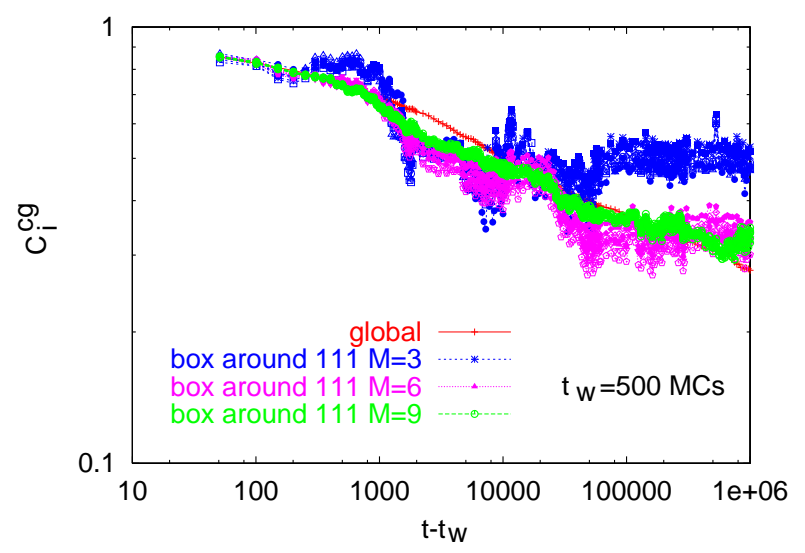

FIG. 12. The local correlation $C_{i}^{c g}\left(t, t_{w}\right)$ against $t-t_{w}$ for $t_{w}=500$ MCs. $L=64$. The three groups of curves correspond to coarse graining volumes $V=(2 M+1)^{3}$ with $M=3,6,9$. Different curves within each group correspond to the 7 sites in the cubic box centered on the site with coordinates $(1,1,1)$. We use a variable coarse-graining time $\tau$ that starts at $\tau=10$ MCs and is multiplied by 5 each time the total time reaches $500 \times 5^{k}$ with $k=1, \ldots$ For comparison we also plot the global correlation $C$.

\section{Distribution functions}

What is the origin of the rather simple scaling of the global correlation for the times explored? Do all local correlations scale in the same way, and as $\approx t / t_{w}$, or is the global scaling the result of the combination of different behaviors on different sites?

In order to explore these questions we first study the time-evolution of the probability distribution function 
(PDF) of local correlations. In Fig. 13 we show the distribution of $C_{i}^{n a}$ 's for several pairs of $t$ and $t_{w}$ with ratios given in the key. Only one disorder realization has been used and the curves are drawn with correlations that have been averaged over $10^{3}$ realizations of the noise. The full distributions scale approximately with $t / t_{w}$. The peak moves towards smaller values of $C_{i}^{n a}$ when the ratio increases (cfr. Fig. 15) and the distribution gets slightly wider. The curves with wider lines correspond to the longest $t_{w}$. For small $t / t_{w}$ a drift with increasing $t_{w}$ leading to a very mild decrease in the height of the peak is visible in the figure. Note that when sufficiently many noise realizations are averaged over, the distribution does not have any weight on negative values of $C_{i}^{n a}$. For even larger values of the ratio $t / t_{w}$ we expect to see a reverse trend in the sense that the distribution has to start getting squeezed around the value $C_{i}^{n a}=0$.

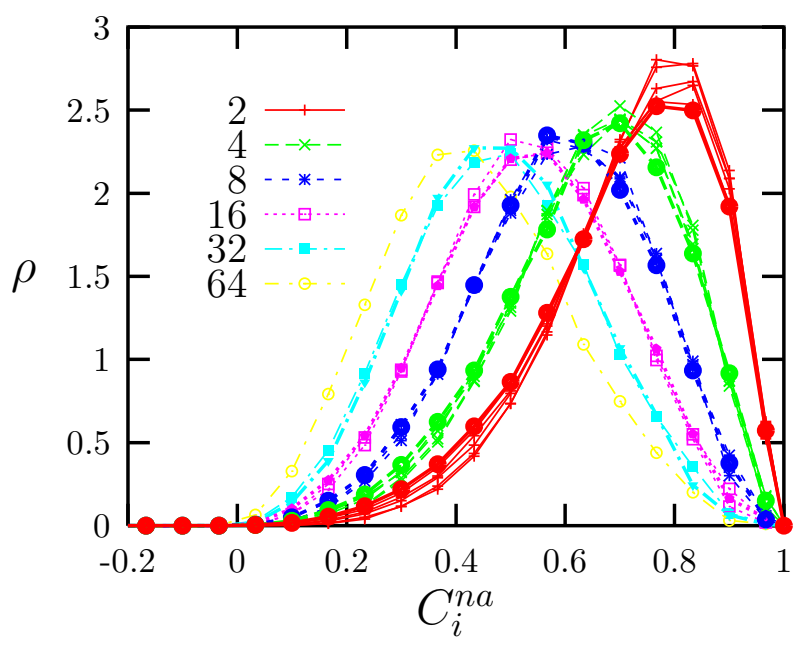

FIG. 13. The distribution of the noise-averaged local correlations $C_{i}^{n a}$ for several pairs of $t$ and $t_{w}$. The waiting-times are $t_{w}=10^{3} \mathrm{MCs}, 2 \times 10^{3} \mathrm{MCs}, 4 \times 10^{3} \mathrm{MCs}, 8 \times 10^{3} \mathrm{MCs}$, $1.6 \times 10^{4} \mathrm{MCs}$, and $3.2 \times 10^{4} \mathrm{MCs}$, and the ratios $t / t_{w}$ are indicated in the key. We averaged over $10^{3}$ realizations of the noise. $\tau_{t}=t / 10, L=64$ and $T=0.7$.

The central part of the distribution is described very well by a Gaussian distribution for intermediate values of $t / t_{w}$ (before the PDF starts to be squeezed on $C_{i}^{n a}=0$ ). Indeed, Fig. 14 shows two Gaussian fits to the numerical PDFs for the ratios $t / t_{w}=8,16$ and $t_{w}=3.2 \times 10^{4}$. Note that since $C_{i}^{n a} \in[0,1]$ the Gaussian fit cannot be perfect. In Sec. IV B we mentioned the interpretation of the local correlations as generating a random surface on the $3 d$ substrate. The approximate Gaussian distribution of the $C_{i}^{n a}$ implies that we can interpret the random surface of noise-averaged correlations as being approximately Gaussian.

The distribution seems to become stable with respect to the number of realizations of the noise, after a large enough number of such realizations. More precisely, we do not see any noticeable variation between the calculated distributions that are averaged over 500 and 1000 thermal histories. We expect this distribution to be self-averaging, i.e., independent of the particular realization of the disorder for large enough systems. We have checked the self-averaging property numerically, and also that, for large enough values of $N$, the distribution becomes size independent.

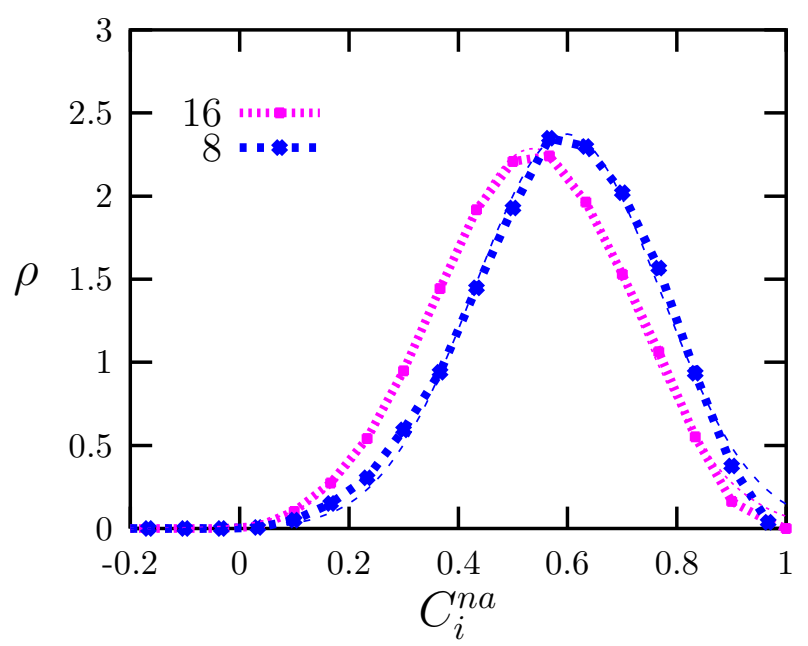

FIG. 14. The distribution of the noise-averaged local correlations $C_{i}^{n a}$ for two ratios $t / t_{w}$ given in the key, $t_{w}=3.2 \times 10^{4}$, and two Gaussian fits. Same parameters as in Fig. 13.

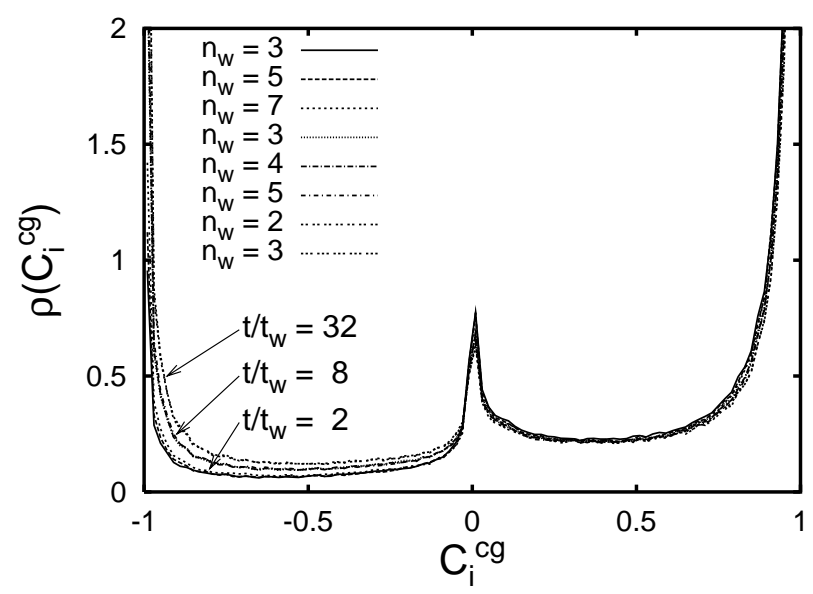

FIG. 15. The distribution of the coarse-grained local correlations $C_{i}^{c g}$ for several pairs of $t$ and $t_{w} . \quad M=0$, $\left[2 M+1=1<\xi\left(t, t_{w}\right)\right] L=32, \tau=10^{3}$ MCs and $T=0.8$. The parameters given in the key determine the waiting-times, $t_{w}=2^{n_{w}} \times 10^{4}$ MCs.

Figure 13 should be compared to Figs. 15-17 in which the distribution of the coarse-grained local correlations, $C_{i}^{c g}$, is shown for several times and different values of the 
coarse-graining volume.

In Fig. 15 the coarse-graining volume has a linear size $2 M+1=1$. Thus, there is no spatial coarse-graining and the only reason why these curves are not simple peaks at -1 and 1 is that the coarse-graining in time, done with $\tau_{t}=10^{3}$ MCs for all times, slightly smooths the data.

In Fig. 16 the coarse-graining volume has a linear size $2 M+1=3$. This distance is slightly shorter than the two-time dependent correlation length, $\xi\left(t, t_{w}\right)$, that we shall define and study in Sec. V. For this amount of coarse-graining the PDF has a nice scaling behavior. The position of the peak is almost independent of the ratio $t / t_{w}$ in this case. Its height diminishes when the ratio $t / t_{w}$ increases and the tail at smaller values of $C_{i}^{c g}$ grows. The scaling with $t / t_{w}$ is rather good in this case.

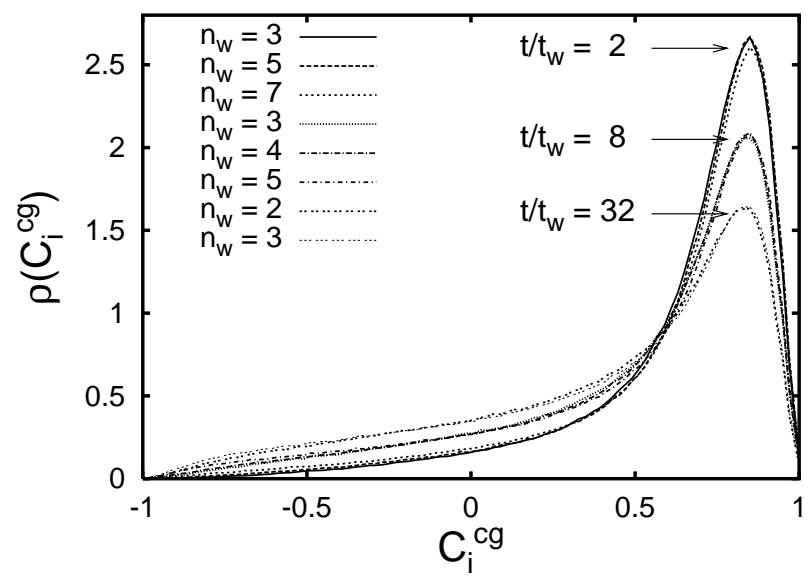

FIG. 16. The distribution of the coarse-grained local correlations $C_{i}^{c g}$ for several pairs of $t$ and $t_{w} . \quad M=1$ $\left[2 M+1=3 \sim \xi\left(t, t_{w}\right)\right], L=32, \tau=10^{3}$ MCs and $T=0.8$. The parameters in the key fix the waiting-times as in Fig. 15.

Finally, in Fig. 17 the coarse-graining volume has a linear size $2 M+1=13$ which is almost half the system size and much larger than the two-time dependent correlation length $\xi\left(t, t_{w}\right)$ for all the waiting and total times studied. The width of the PDFs has been considerably reduced with respect to the previous case. In particular, the PDF does not have any weight on negative values, as opposed to what is shown in Fig. 16. It has also become quite symmetric, centered at the global value which is also approximately the average value of this PDF. Reasonably, the distributions drift towards smaller values of the correlations when the value of $t / t_{w}$ increases. Moreover, the scaling with $t / t_{w}$ worsens with too much coarse-graining, as is to be expected. Using such a large coarse-graining volume one approaches the limit in which the distribution becomes a delta-function at the global value. The $t / t_{w}$ scaling is only an approximation to the true scaling, see Fig. 9. In order to improve the fit one should eliminate the contributions to the stationary decay but this is not easy to do at the level of the full distribution.

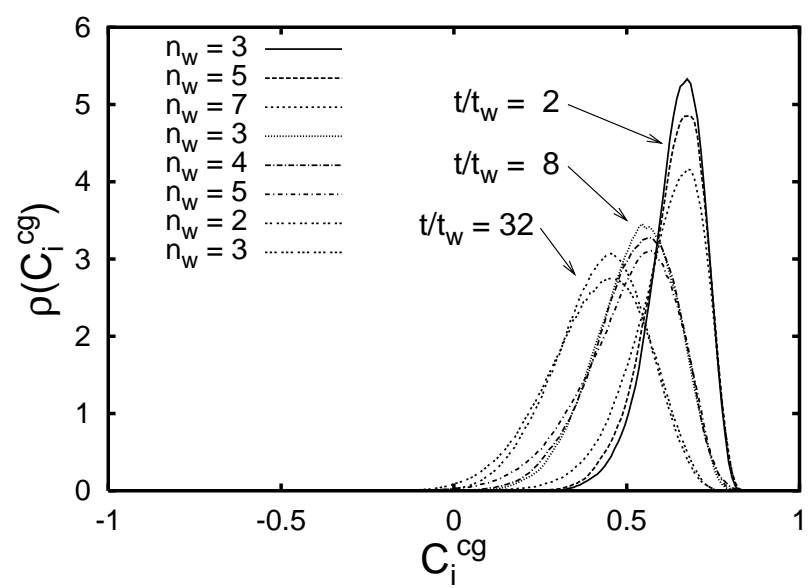

FIG. 17. The distribution of the coarse-grained local correlations $C_{i}^{c g}$ for several pairs of $t$ and $t_{w}$. $M=6$ $\left[2 M+1 \gg \xi\left(t, t_{w}\right)\right], L=32, \tau=10^{3} \operatorname{MCs}$ and $T=0.8$. The parameters in the key fix the waiting-times as in Fig. 15.

It is clear that for a given disorder and thermal history this distribution approaches a delta function on the time-dependent global value of the correlation when the coarse-graining volume reaches the size of the system. Even in this limit, for finite $N$ we still have sample-tosample and noise-to-noise fluctuations and hence a nontrivial distribution of these points.

To conclude, the noise-averaged PDF and the coarsegrained PDF approximately scale with $t / t_{w}$ for long waiting-times when $2 M+1 \sim \xi\left(t, t_{w}\right)$. However, even if for a given ratio $t / t_{w}$ the mean of the distributions coincides (yielding the global value in both cases), the form of the two PDFs differs at equal $t / t_{w}$. This is most clearly seen by comparing, for instance, the curves for $t / t_{w}=32$ in Figs. 13 and 16. The most distinctive difference is given by the persistence of a peak in $\rho\left(C^{c g}\right)$ that is centered on a stable value (although its height decreases with increasing $\left.t / t_{w}\right)$. Moreover, the PDF of noise-averaged correlations is approximately Gaussian for intermediate $t / t_{w}$ values while the PDF of coarse-grained two-time local correlations is not when $2 M+1 \sim \xi\left(t, t_{w}\right)$. For larger values of the linear size of the coarse-graining volume the PDF looks more Gaussian, see Fig. 17, at least in its central part. We have observed the same features at other temperatures.

We end this subsection by pointing out that the probability distributions for noise-averaged and coarse-grained quantities can be interpreted as, respectively, probabilities of averages and averages of probabilities. To see this relation, consider a quantity that is noise averaged after being coarse grained, and its probability density is $\rho\left(\left\langle C_{i}^{c g}\right\rangle\right)$. In the limit when the coarse graining volume is a single spin $(M=0)$, we recover $\rho\left(C_{i}^{n a}\right)$, us- 
ing $C_{i}^{n a}=\left\langle C_{i}\right\rangle$. Now, for a large enough total system size, the probability distribution for coarse-grained quantities $\rho\left(C_{i}^{c g}\right)$ becomes self-averaging, $\rho\left(C_{i}^{c g}\right) \rightarrow\left\langle\rho\left(C_{i}^{c g}\right)\right\rangle$. Hence, the differences between the noise-averaged and coarse-grained probabilities derive from the fact that $\rho\left(\left\langle C_{i}^{c g}\right\rangle\right) \neq\left\langle\rho\left(C_{i}^{c g}\right)\right\rangle$.

\section{E. Scaling of noise-averaged local correlators}

We have just shown that the even if the probability distributions of noise-averaged and coarse-grained local correlations take different forms, they scale approximately as $t / t_{w}$. This does not mean, however, that each site has a local correlation scaling with $t / t_{w}$. Equations (17) and (18) can now be put to the test by studying the decay in time of the individual local correlations $C_{i}^{n a}$ and $C_{i}^{c g}$.

A simple way to test Eq. (17) is to plot the values of the local correlations at different sites for different pairs $\left(t, t_{w}\right)$ such that their ratio, $t / t_{w}$, is held fixed. If the hypothesis is correct, for a given site, its correlation must take a very similar value for all $t_{w}$ 's. Figure 18 shows this test for a $3 d$ EA model of linear size $L=32$ at $T=0.7$. The average involves $10^{3}$ noise realizations. In the three panels we used different values of the ratio $t / t_{w}$ as labelled. The points represent the values of the noise averaged local correlations at sites in four rows in the cube. More precisely, the discrete points on the axis labelled "site" correspond to $(x=0, y=0, z=0, \ldots, L-1)$, $(x=0, y=1, z=0, \ldots, L-1),(x=0, y=2, z=$ $0, \ldots, L-1)$ and $(x=0, y=3, z=0, \ldots, L-1)$ ordered in this way. This means that, for example, the values site $=0$, site $=32$ and site $=64$ are nearby sites on the lattice, site $=0$ being $(x=0, y=0, z=0)$, site $=32$ being $(x=0, y=1, z=0)$ and site $=64$ being $(x=0, y=2, z=0)$. This explains the approximate periodicity in the data: it indicates that nearby sites have a rather similar behavior as most clearly indicated by staring at the points with rather small values of $C_{i}^{n a}$. The lines are added as guides to the eye. The figure shows that the noise averaged correlations satisfy the hypothesis in Eq. (17) for this range of times. The site-dependent external function $f_{i}$ in Eq. (17) reflects the fingerprint of disorder.

Interestingly enough, even running at different temperatures (with the same seeds for the thermal noise) the individual evolution of the sites is still very similar (see Fig. 19). This issue deserves further investigation since it might be very useful in helping to explain the intriguing memory and rejuvenation effects seen in the dynamics of spin-glasses when temperature is modified. Nevertheless, we shall not expand on this topic here.
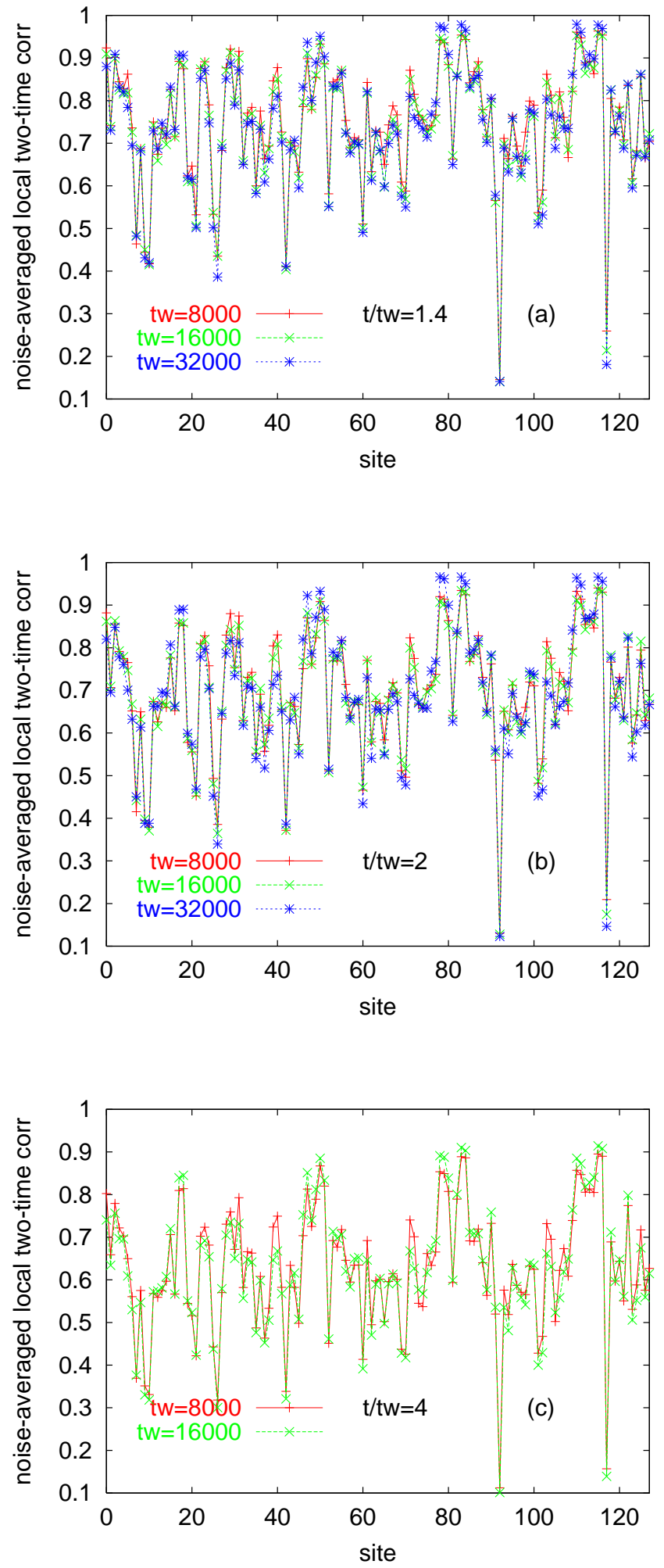

FIG. 18. The fingerprint of disorder. The noise-averaged local correlation on 128 sites ordered along four adjacent rows for three choices of the ratio $t / t_{w}$ indicated as a label in each panel. $L=32, T=0.7, \tau_{t}=t / 10$. The curves for different $t_{w}$ 's fall on top of each other showing that $C_{i}^{n a}$ scales as in Eq. (17). 


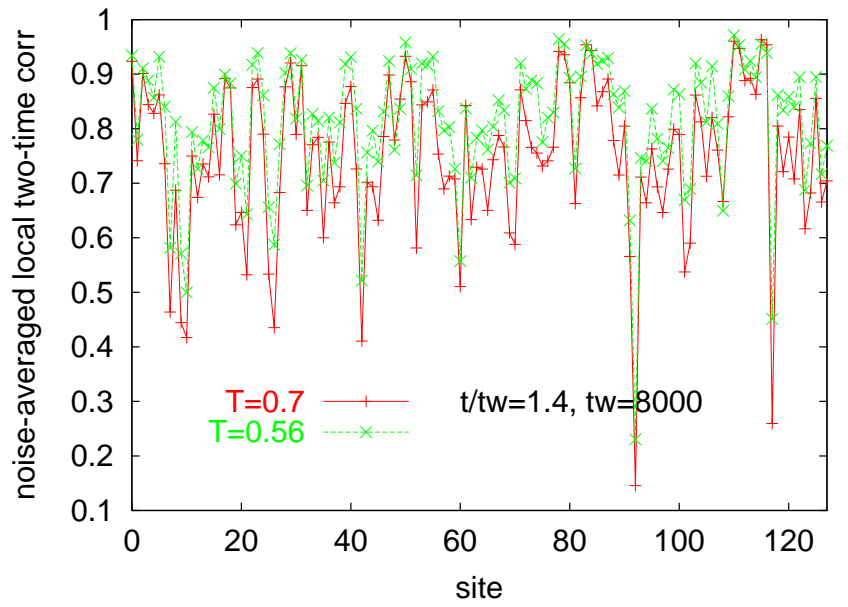

FIG. 19. Competition between temperature and quenched disorder. The noise-averaged local correlation on adjacent 128 sites for two temperatures, $T=0.56$ and $T=0.7$. The time ratio is $t / t_{w}=1.4, t_{w}=8 \times 10^{3}$ MCs and $\tau_{t}=t / 10$. The two sets of data are surprisingly similar.

\section{F. Scaling of coarse-grained local correlators}

We can now analyze the coarse-grained local correlations in the same manner as the noise-averaged ones.

First, in Fig. 20 we show $C_{i}^{c g}$ on 128 sites chosen as in Fig. 18 (see the text above) for three choices of $t / t_{w}=$ 2,4 , and 64 at $t_{w}=10^{3}$ MCs. The coarse-graining volume is small, $M=1$. The curves for different $t_{w}$ 's do not scale exactly since the noise induces fluctuations in addition to those that originate from the quenched disorder. $C_{i}^{c g}$ does not scale as in Eq. (17) for any of the choices of the ratio $t / t_{w}$.

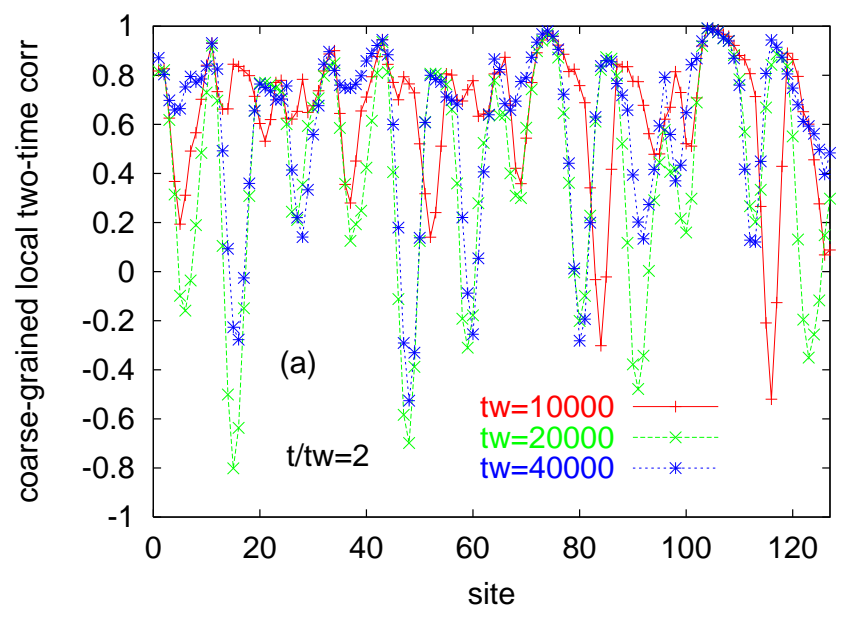

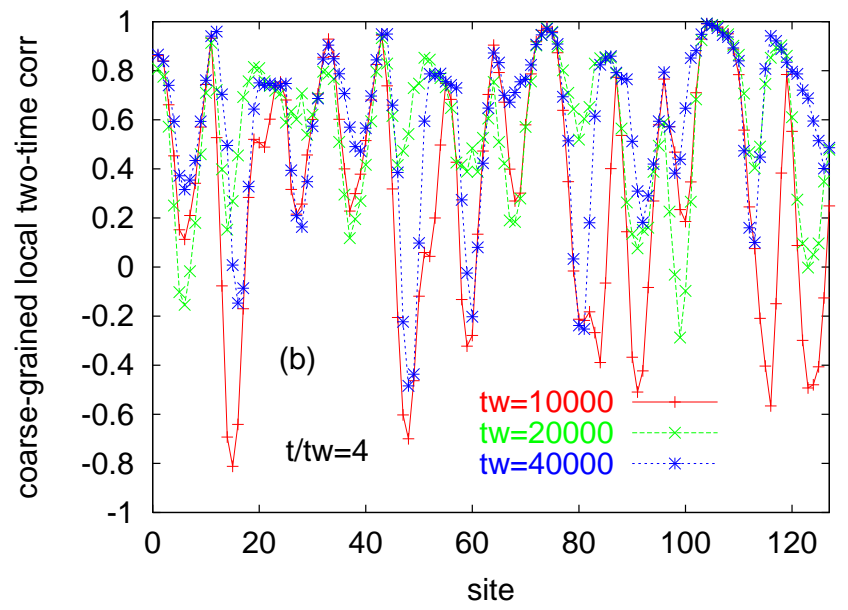

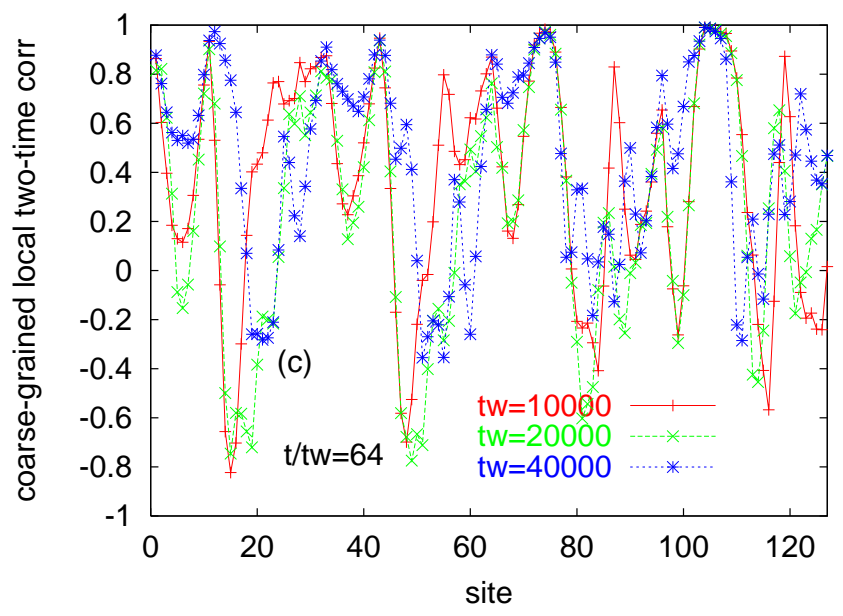

FIG. 20. Test of $t / t_{w}$ scaling in the coarse-grained correlation. Values of this quantity on 128 adjacent sites for three choices of the ratio $t / t_{w}$ indicated in each panel. $M=1$, $L=32, T=0.8$, and $\tau_{t}=t / 10$.

Notice that the curves obtained for different pairs of time $\left(t, t_{w}\right)$, even though the ratio $t / t_{w}$ is fixed, cross each other at many points. Therefore, it is always possible to find sites $i$ and $j$ at opposite sides of the crossing points for which $C_{i}^{c g}>C_{j}^{c g}$ for one pair of $\left(t, t_{w}\right)$, but $C_{i}^{c g}<C_{j}^{c g}$ for another. In other words, the relative age (as measured using the correlation value) between two sites $i$ and $j$ in the sample is not static, but fluctuates as a function of time. These are exactly the sorpassi that we described in Sec. IIE. Once again, notice that this is in sharp contrast with the scaling Eq. (17), where the relative age between all sites in the sample keeps a constant, static, relative rank.

In Fig. 21 we compare the coarse-grained and noiseaveraged local correlations for two systems with the same quenched disorder, evolved at the same temperature. We plot the local correlations on one row of the $3 d$ cube corresponding to $(x=0, y=0, z=0, \ldots, L-1)$, with 
$L=32$. The temperature is $T=0.7$. In all panels we plot the noise-averaged data for three $t_{w}$ 's, $t_{w}=8 \times 10^{3}$ MCs, $1.6 \times 10^{4}$ MCs and $3.2 \times 10^{4}$ MCs, with crosses and the coarse-grained data, for the same waiting-times, with open squares $\left(t_{w}=8 \times 10^{3} \mathrm{MCs}\right)$, open circles $\left(t_{w}=1.6 \times 10^{4} \mathrm{MCs}\right)$ and dark squares $\left(t_{w}=3.2 \times 10^{4}\right.$ MCs). The lines joining the points are a guide to the eye. The ratio between $t$ and $t_{w}$ is always $t / t_{w}=1.4$ but similar results are obtained for other choices. In different panels in the figure we use different values of $M, M=0$ (a), $M=1$ (b), $M=3$ (c), and $M=5$ (d).

The first result from these figures is that, as already shown in Fig. 18, the noise-averaged data scale as in Eq. (17). Instead, the coarse-grained data do not scale in this way for any of the values of $M$.

Let us now discuss in detail the effect of coarsegraining. In panel a) we compare the noise-averaged data to the results of a single run without coarse-graining $(M=0)$. We see that the $t / t_{w}$ scaling does not hold for the curves with coarse-grained data, but the values of the local correlations are very much influenced by the disorder. For instance, observe the low values simultaneously taken by the correlation at $\mathrm{site}=10$ for the noiseaveraged quantity and also in the single thermal history runs.

In panel b) we use a minimum volume $V=(2 M+1)^{3}=$ $3^{3}$. We see that the "surface" created by the coarsegrained data has been smoothed with respect to the case $M=0$ shown in panel a) but there remains a memory of the underlying quenched disorder. The coarse-graining does not help improve the $t / t_{w}$ scaling.

This trend is even clearer in panels c) and d) where we use $M=3$ and $M=5$, respectively. The $t / t_{w}$ scaling is clearly broken as the surfaces become smoother and smoother when $M$ increases. When $M=5$ the surface is almost totally flat and the fingerprint of disorder has been washed out by coarse-graining. However, there are still soft local fluctuations that break the $t / t_{w}$ scaling.

The conclusion we draw from these plots, and from the theoretical discussion in Sec. III, is that for a truly infinite system, $N=L^{3} \rightarrow \infty$, we expect to find that when times diverge, $t, t_{w} \rightarrow \infty$ with $C\left(t, t_{w}\right)$ fixed to a chosen value, say $C\left(t, t_{w}\right)=\mathrm{C}$, thus selecting the correlation scale, coarse-graining over a sufficiently large volume erases the fingerprint of disorder whilst still allowing for fluctuations that change the reparametrization locally, $h(t) \rightarrow h_{i}(t)=h(t)+\delta h_{i}(t)$. This argument implies that hypothesis (18) should describe the data in this limit. In Sec. V we shall further discuss the implications of coarsegraining.

The final test of this hypothesis would be to plot the value of the correlation on each site for different pairs of times such that $h_{i}(t) / h_{i}\left(t_{w}\right)$ is held fixed. If the hypothesis in Eq. (18) holds, the surface of the resulting figure should be flat.
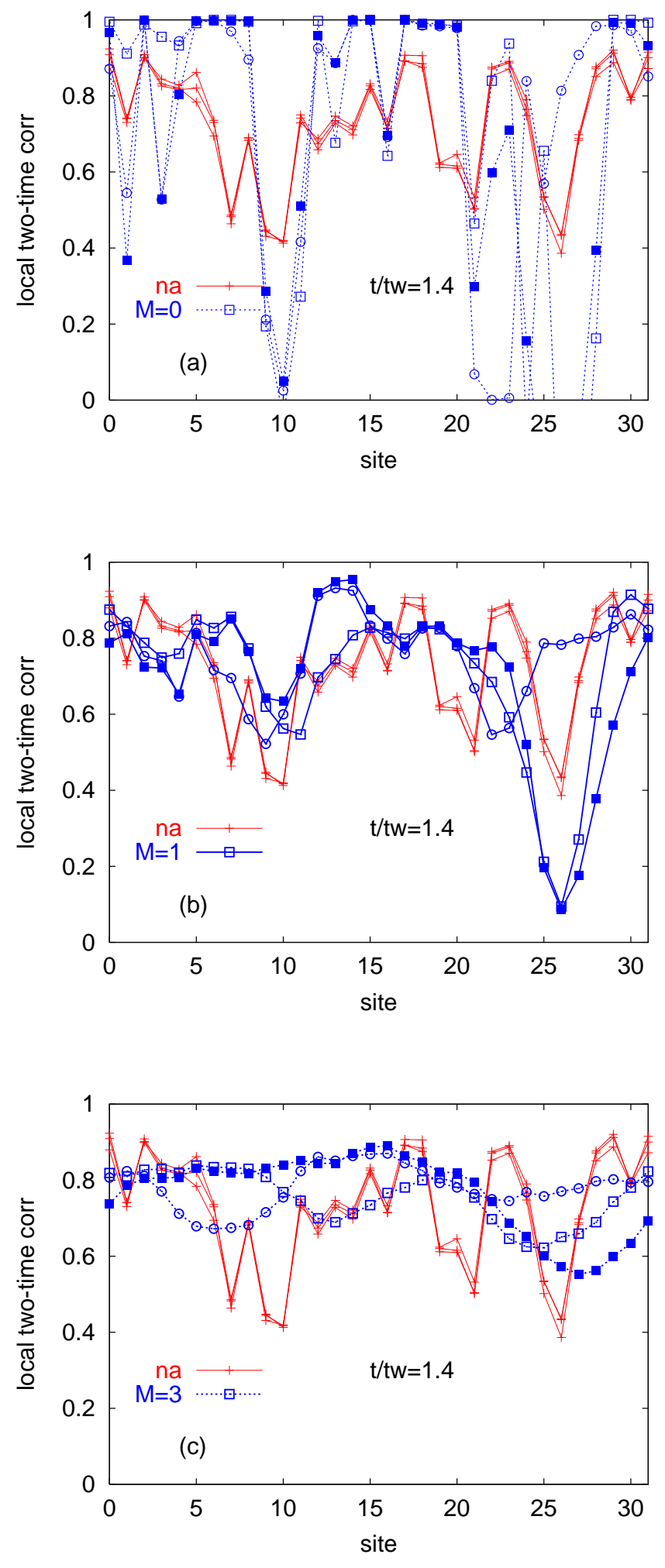


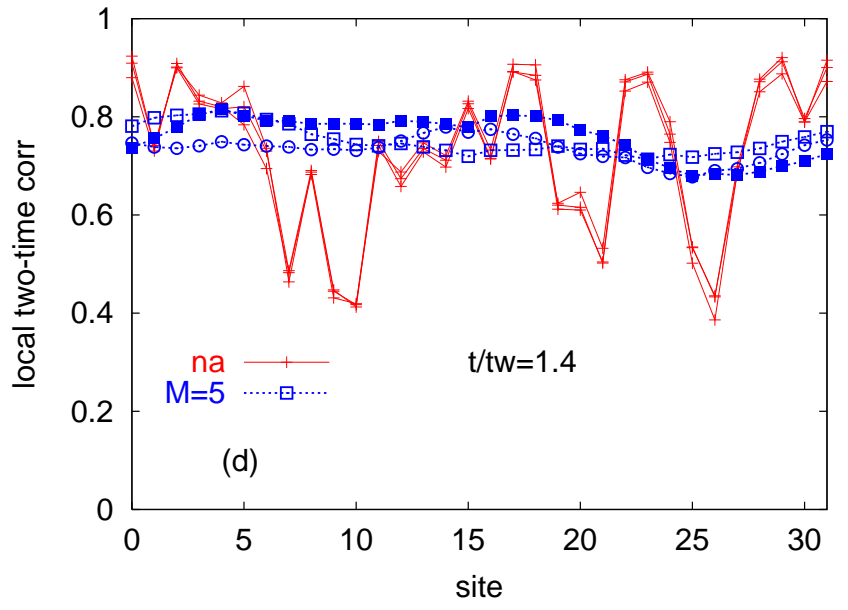

FIG. 21. Comparison between noise-averaged and coarse-grained local correlations. The three curves in each group correspond to three waiting-times. $t_{w}=8 \times 10^{3}$ MCs (open squares), $t_{w}=1.6 \times 10^{4}$ MCs (open circles) and $t_{w}=3.2 \times 10^{4}$ MCs (filled squares) for the coarse-grained data. The coarse-graining volume is indicated in the labels. $L=32, T=0.7$. The coarse-graining times is $\tau=8 \times 10^{2}$ MCs when $t_{w}=8 \times 10^{3}$ MCs and is multiplied by 2 when the time is doubled.

An equivalent way to check hypothesis (18) is to plot the decay of the local correlations on several sites, for several chosen values of $t_{w}$ and all subsequent $t$, against different ratios $\lambda_{i}=h_{i}(t) / h_{i}\left(t_{w}\right)$. For each site $i$ the choice of an adequate scaling function $h_{i}(t)$ should lead to a collapse of each of the local correlations $C_{i}\left(t, t_{w}\right)$ corresponding to different $t_{w}$ 's. Moreover, if the (in principle site-dependent) master curves, $f_{i}$, thus obtained are all identical, $f_{i}=f$ for all $i$, then the conjecture in Eq. (18) is satisfied.

The careful implementation of these checks is rather tedious since one needs an independent inspection of the dynamics of each site in the sample. We take the flattening of the curves in Fig. 21 for increasing $M$ as strong evidence for the scaling in Eq. (18) in the "scaling limit" defined in Sec. VB.

\section{G. Effect of coarse-graining on already noise-averaged quantities}

In Fig. 22 we coarse-grain the already noise-averaged data for the local correlation. When $M=3$ the surface is already quite flat (and $t / t_{w}$ dependent). This is to be compared with the the data in Fig. 21c) for one run coarse-grained over the same volume. We see how the fingerprint of disorder progressively disappears with more coarse-graining.

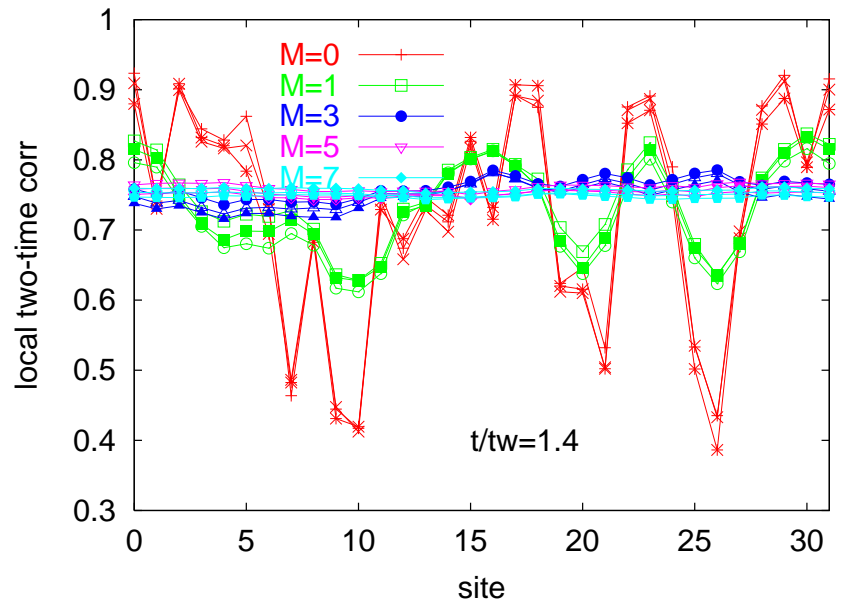

FIG. 22. Effect of coarse-graining on the local noise - averaged data. The parameters are as in Fig. 21

\section{TWO-TIME DEPENDENT CORRELATION LENGTH}

In this section we define a correlation length from the study of the spatial fluctuations of the two-time local correlators. We also discuss the interplay between times and length-scales in the analysis of our data.

\section{A. Definition}

The presence of a Goldstone mode in the dynamics gives rise to a specific form for the spatial correlations of the fluctuating two-time fields $Q_{i}^{\alpha \beta}\left(t, t_{w}\right)$. In the asymptotic limit, the $\mathrm{R} p \mathrm{G}$ invariance implies a true Goldstone, or zero mass, mode. Therefore, the spatial correlations in the fluctuations should show a power law decay $\sim 1 / r$ in $3 d$. However, as mentioned before, we know that for any finite time, the $R p G$ invariance is explicitly broken by irrelevant terms that play the role of symmetry breaking fields. As in the case of magnons in the presence of a weak external magnetic field, the Goldstone mode in the dynamics acquires a small mass, which vanishes in the asymptotic limit of small symmetry breaking field, i.e. in the long time limit. Therefore, we expect that in a simulation, the local two-time quantities will exhibit a finite correlation length for their fluctuations. Fixing the relation between $t$ and $t_{w}$ to have, say, a given global correlation, one should find a correlation length $\xi\left(t, t_{w}\right)$ that increases monotonically for increasing $t_{w}$. Equivalently, the mass $m\left(t, t_{w}\right)=1 / \xi\left(t, t_{w}\right)$ should be a monotonically decreasing function of the times [66].

Most of the results about the finite correlation length that we present were obtained for a $3 d$ EA model with linear size $L=32$ evolving at $T=0.8$. We considered 64 
realizations of the disorder. The length of the simulation was $8.192 \times 10^{7}$ MCs.

The two-time quantities considered are the local twotime correlations $C_{\vec{r}_{i}}\left(t, t_{w}\right) \equiv C_{i}\left(t, t_{w}\right)=\bar{s}_{i}(t) \bar{s}_{i}\left(t_{w}\right)$, where the $\bar{s}_{i}(t)$ are site magnetizations time averaged over $\tau=10^{3}$ MCs. The vector $\vec{r}_{i}$ is the position of site $i$ with respect to a chosen origin of coordinates.

We define a spatial correlator

$$
\tilde{A}\left(r ; t, t_{w}\right) \equiv\left[\frac{1}{N} \sum_{i} C_{\vec{r}_{i}}\left(t, t_{w}\right) C_{\vec{r}_{i}+\vec{r}}\left(t, t_{w}\right)\right]
$$

that is averaged over disorder realizations but is not averaged over the noise. Since the problem is isotropic on average we expect the r.h.s. to depend only on the modulus of the distance between the positions of the spins considered, $r \equiv|\vec{r}|$. Thus we write $r$ in the argument of $\tilde{A}$. $N=L^{3}$ is the total number of spins in the sample. As $r \rightarrow \infty$ we expect that this correlator will approach its disconnected part:

$$
\begin{aligned}
\tilde{A}_{\infty}\left(t, t_{w}\right) & \equiv \lim _{r \rightarrow \infty} \tilde{A}\left(r ; t, t_{w}\right)=\left[\left(\frac{1}{N} \sum_{i} C_{\vec{r}_{i}}\left(t, t_{w}\right)\right)^{2}\right] \\
& =\left[C\left(t, t_{w}\right)^{2}\right],
\end{aligned}
$$

with $C\left(t, t_{w}\right)$ the global correlation.

In order to define a correlator that has a spatially asymptotic value that is independent of the times $t$ and $t_{w}$ we define the normalized correlator:

$$
A\left(r ; t, t_{w}\right) \equiv \frac{\tilde{A}\left(r ; t, t_{w}\right)}{\tilde{A}_{\infty}\left(t, t_{w}\right)}
$$

which tends to unity in the limit $r \rightarrow \infty$. Therefore, the connected part of $A$ is simply $A-1$. Note that the equal site correlator $A\left(r=0 ; t, t_{w}\right)$ is very close to $1 / \tilde{A}_{\infty}\left(t, t_{w}\right)$ since the zero-distance correlator, $\tilde{A}\left(r=0, t, t_{w}\right)$, involves $\bar{s}_{i}{ }^{2}(t)$ and $\bar{s}_{i}{ }^{2}\left(t_{w}\right)$ that for short coarse-graining times $\tau$ and long times $t$ and $t_{w}$ are approximately equal to 1 . For any $t_{w}$ the denominator $\tilde{A}_{\infty}\left(t, t_{w}\right)=\left[C\left(t, t_{w}\right)^{2}\right]$ vanishes in the limit $t \gg t_{w}$. Consequently, as it is defined, the correlator $A\left(r ; t, t_{w}\right)$ evaluated at zero spatial distance diverges with increasing $t$ for any value of $t_{w}$. At equal times $A$ is approximately 1 for all distances $r$; the reason for this result is again the fact that $\bar{s}_{i}{ }^{2}(t) \approx 1$.

One can also define a correlator that is forced to evolve, for all pairs of times $t$ and $t_{w}$, between 1 at zero distance and 0 for infinitely separated distances. Indeed,

$$
B\left(r ; t, t_{w}\right) \equiv \frac{\tilde{A}\left(r ; t, t_{w}\right)-\tilde{A}_{\infty}\left(t, t_{w}\right)}{\tilde{A}\left(r=0 ; t, t_{w}\right)-\tilde{A}_{\infty}\left(t, t_{w}\right)}
$$

satisfies these requirements.

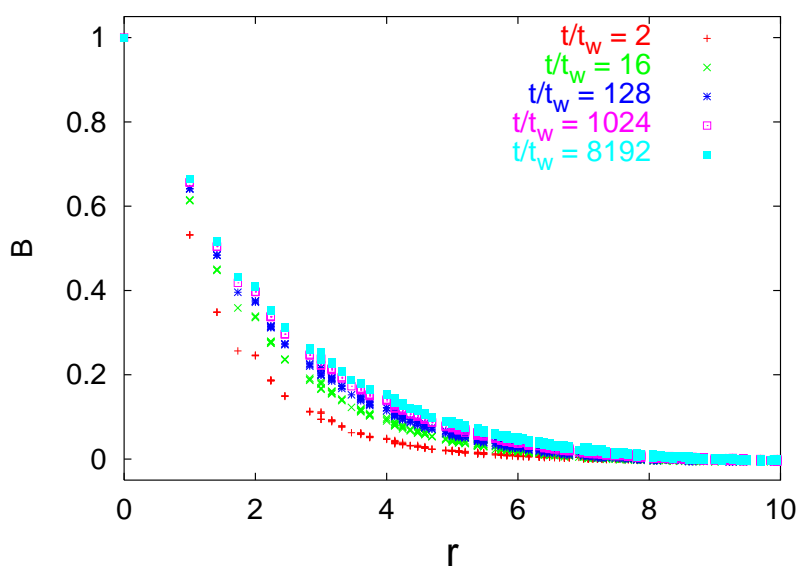

FIG. 23. Spatial correlations $B\left(r ; t, t_{w}\right)$ against distance $r$ for $t_{w}=10^{4}$ MCs and $t / t_{w}=2,16,128,1024$, and 8192 .

In Fig. 23, we show the space dependence of $B\left(r ; t, t_{w}\right)$, for various ratios $t / t_{w}$. In all cases we observe that the correlation decays rapidly, consistent with an exponential decay, for $r<6$ lattice spacings. To make this more apparent, we show a semilog plot for the same $t / t_{w}$ ratios in Fig. 24.

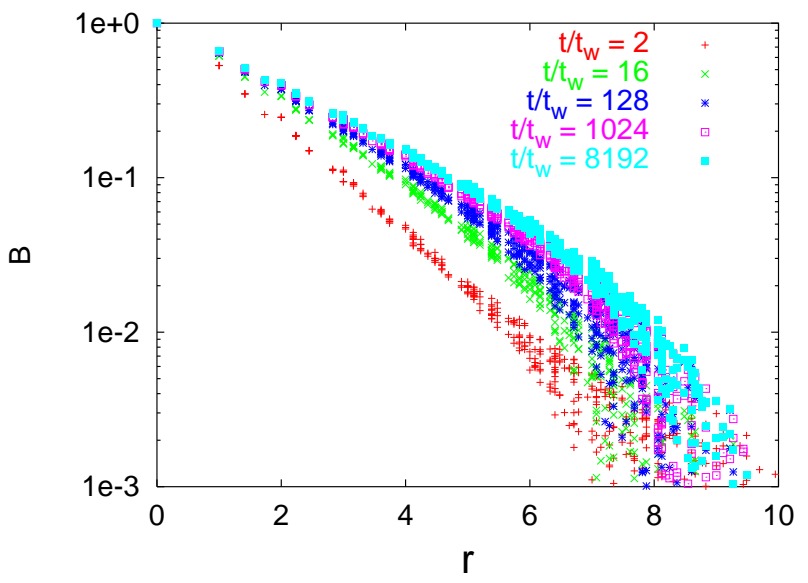

FIG. 24. Spatial correlations $B\left(r ; t, t_{w}\right)$ against distance $r$ in a semi-logartihmic scale for $t_{w}=10^{4} \mathrm{MCs}$ and $t / t_{w}=2$, $16,128,1024$, and 8192.

In the previous two figures, the horizontal axis represents the distance $r$. One point is plotted for each vector $\vec{r}$, therefore many points with similar $r$ but corresponding to different directions for $\vec{r}$ appear close to each other. The fact that in the figures the corresponding values of $B$ are also close together indicates that the spatial correlations are indeed spatially isotropic, as expected. 


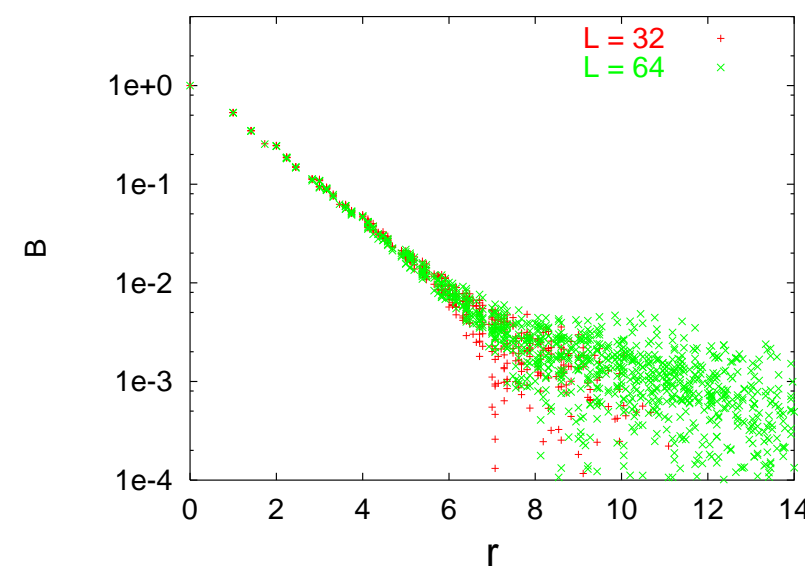

FIG. 25. Comparison between the spatial dependence of $B\left(r ; t, t_{w}\right)$ for $L=32$ and $L=64$, with $t_{w}=10^{4} \mathrm{MCs}$ and $t / t_{w}=2$.

In Fig. 25, we show the decay of the correlation for two system sizes $L=32$ and $L=64$. We observe that the decay is roughly independent of the system size, up to distances of the order of $r \sim 6$. One notices that the trend of the points, after this limit, is to bend downwards for $L=32$ and to bend upwards for $L=64 . r \sim 6$ is also the distance upto which the exponential fit [see Eq. (67) and Fig. 26] is very good. (In this figure, $t_{w}=10^{4} \mathrm{MCs}$ and $t / t_{w}=2$.)

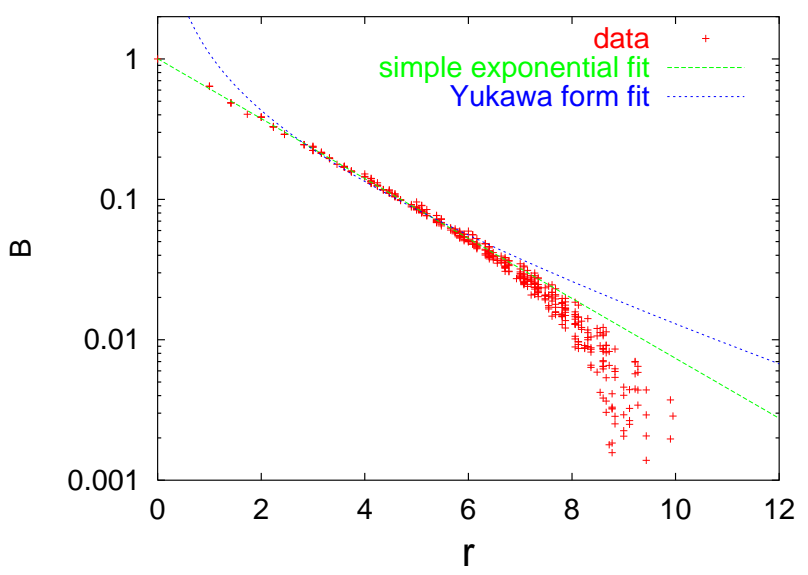

FIG. 26. Example of fit with an exponentially decaying function given in Eq. (67) and a Yukawa type of correlator in $3 d$ given in Eq. (68) (see the text for details). The data correspond to $t_{w}=3.2 \times 10^{5} \mathrm{MCs}, t=2.56 \times 10^{6} \mathrm{MCs}$. The data were fitted in the interval $r \in[1.5,6.0]$.

In Fig. 26, we show a typical example of a fit of the correlation to exponentially decaying functions. Two possible fits are displayed, one with the simple exponential form

$$
B_{\exp }\left(r ; t, t_{w}\right)=D\left(t, t_{w}\right) e^{-m\left(t, t_{w}\right) r},
$$

the other with the Yukawa form

$$
B_{\text {Yuk }}\left(r ; t, t_{w}\right)=D\left(t, t_{w}\right) \frac{e^{-m\left(t, t_{w}\right) r}}{r} .
$$

The second form is the 3-dimensional Fourier transform of the massive correlator $1 /\left(k^{2}+m^{2}\right)$, and one would naively expect this form to provide the best fit. However, on deeper examination, it is necessary to take into account the form of the fluctuations, as discussed in Sec IIID (see Eq. 56), and in particular the fact that $C\left(\vec{r}, t, t_{w}\right)$ appears to depend exponentially on the two fluctuating fields $\phi\left(\vec{r}, t_{i}\right)\left(t_{i}=t, t_{w}\right)$. Since the fluctuations in $C\left(\vec{r}, t, t_{w}\right)$ are significant, even if the propagator for each reparametrization fields $\phi\left(\vec{r}, t_{i}\right)$ had a simple Yukawa form, the form of $B\left(r ; t, t_{w}\right)$ is in principle highly nontrivial, and in particular the value of the mass $m\left(t, t_{w}\right)$ is not easy to predict.

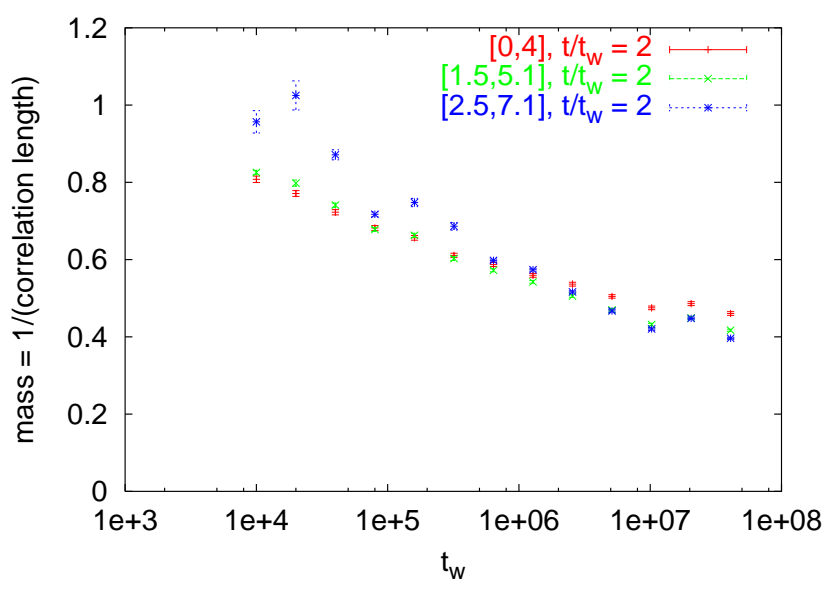

FIG. 27. Dependence of the fitted values of the mass on the interval chosen for the fit. Comparison between values of the mass obtained by fitting in the intervals $r \in[0.0,4.0]$, $r \in[1.5,5.1]$, and $r \in[2.5,7.1]$, displayed as a function of $t_{w}$. In all cases, $L=32$, and $t / t_{w}=2$.

For the range of times and system sizes achieved in our simulations, it turns out that both the simple exponential and the Yukawa forms provide good fits for intermediate values of $r$, but the first one consistently has a better $\chi^{2}$, and it consistently provides a better extrapolation outside the fitting interval. For larger values of $r$, finite size effects seem to play an important role, producing an even steeper decay of the correlations. In principle there is no reason to expect the fit to be good for small values of $r$. However, the simple exponential form actually does provide a good fit down to $r=0$. In what follows, all the values of the mass (equivalently, the inverse correlation length) are obtained by using a simple exponential fit in the interval $[0,4]$. Each fit has 52 degrees of freedom, and typical $\chi^{2}$ per degree of freedom are of the order of $10^{-3}$. From the definition in Eq. (66), we know that $B(r=$ $\left.0 ; t, t_{w}\right)=1$. The fitting functions that we use do not 
need to have exactly this value at the origin, but it turns out that, for the simple exponential form, $D\left(t, t_{w}\right) \approx 1$ within an error of the order of $10 \%$.

Figure 27 displays a comparison of results obtained by using different fitting intervals. Changing the fitting interval does somewhat affect the results of the fits, but the trends are consistent, and the interval chosen $(r \in$ $[0.0,4.0])$ seems to be the one that minimizes the noise in the mass versus time curve.

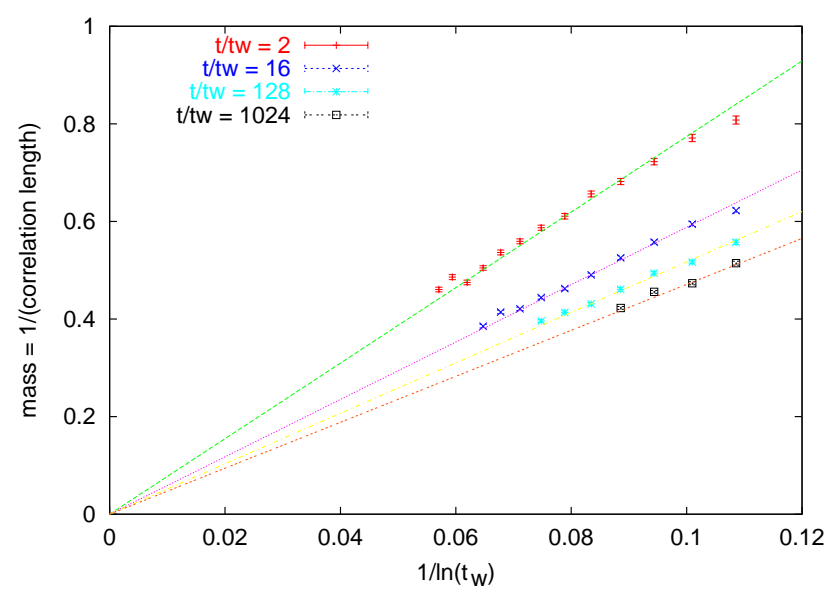

FIG. 28. Mass (inverse correlation length) as a function of the waiting-time. Each set of points corresponds to a fixed ratio $t / t_{w}$. The horizontal axis corresponds to $1 / \ln t_{w}$.

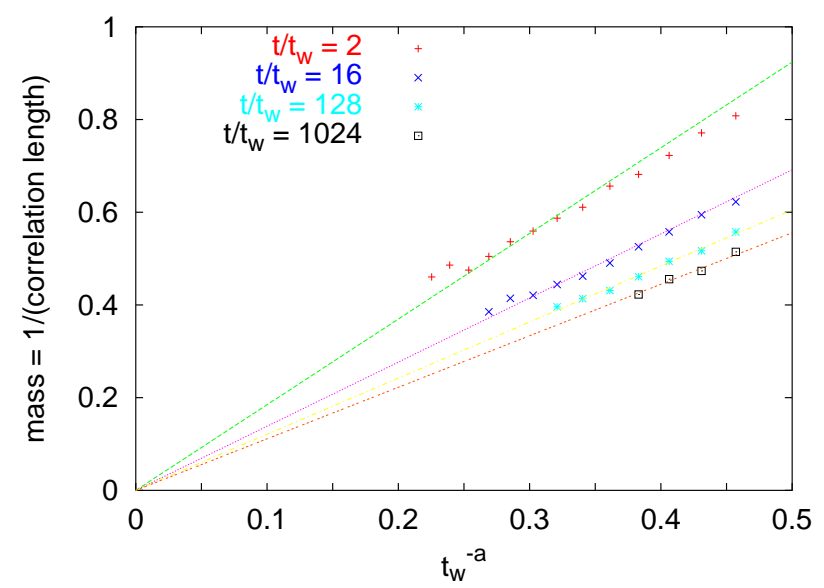

FIG. 29. Mass (inverse correlation length) as a function of the waiting-time. Each set of points corresponds to a fixed ratio $t / t_{w}$. The horizontal axis corresponds to $t_{w}{ }^{-a}$ with $a=0.085$.

In Figs. 28 and 29, we show the two-time dependence of the mass in our simulations. Each set of points corresponds to a fixed ratio $t / t_{w}$. We attempt two fits of the mass as a function of $t_{w}$. In Fig. 28 we show the fit $m\left(t, t_{w}\right) \equiv 1 / \xi\left(t, t_{w}\right) \approx m_{0}\left(t / t_{w}\right) / \ln t_{w}$. In Fig. 29, we show the fit $m\left(t, t_{w}\right) \equiv 1 / \xi\left(t, t_{w}\right) \approx m_{0}\left(t / t_{w}\right)\left(t_{w} / t_{0}\right)^{-a}$, with $a=0.085$. The simulation results are consistent with both fitting forms. Due to the very slow decay of the mass with $t_{w}$, it is not possible to distinguish between the two fits. Another important feature of the data is revealed by these figures: the correlation length increases with increasing ratio $t / t_{w}$, at fixed $t_{w}$. One must note, though, that the variation of the mass with the ratio $t / t_{w}$ is also quite mild.

An intriguing fact in our results is that the values of the correlation length obtained are extremely short. Note that Figs. 28 and 29 show a variation of the mass between 0.4 and 0.8 that corresponds to lengths between 1.25 and 2.5 lattice spacings. As shown in Fig. 30 these values change quite a bit if one uses instead a fitting procedure with the Yukawa form in Eq. (68). As mentioned before, the simple exponential form provides a better fit for the range of distances accessible in our simulations. However, this conclusion might in principle change if much larger sizes were simulated. (See, e.g. Fig. 25, where the data for $L=64$ seem to deviate above the simple exponential fit for longer distances, which would be consistent with a different functional form for $B$ and possibly a smaller value of the mass.) The big difference in the mass values for the two fits indicates that any quantitative results about the precise value of the mass have to be taken with caution, and that our conclusions should be restricted to indicate general trends in the data. Based on these considerations we shall postulate, somewhat arbitrarily, that the correlation lengths for the times explored in the rest of this paper are in between the two fits and of the order of 3 to 5 lattice spacings.

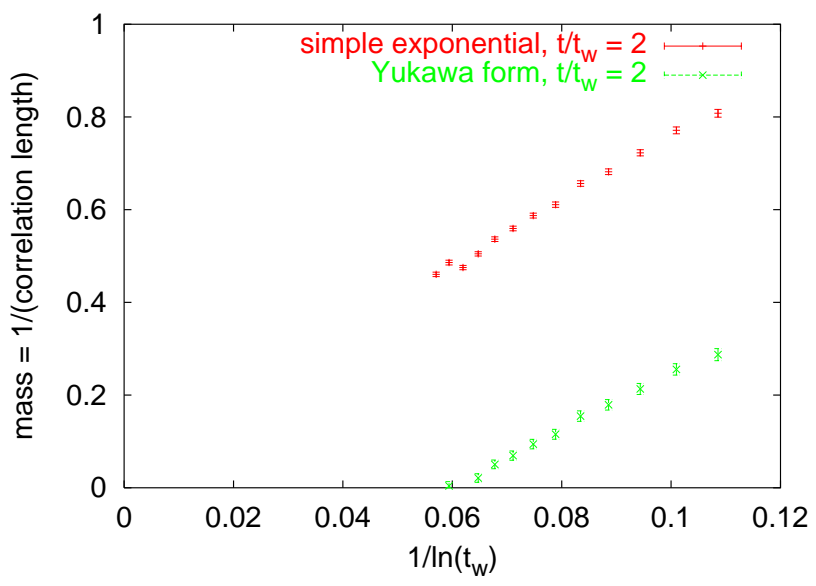

FIG. 30. Comparison between the values of the masses obtained with the exponential fit given in Eq. (67) and the fit with the Yukawa form given in Eq. (68). In both cases $L=32$, $t / t_{w}=2$.

Let us discuss a qualitative picture for describing the two-time dependence of the $m\left(t, t_{w}\right)$ suggested by the random surface action of Sec. IIID. Although $m\left(t, t_{w}\right)$ depends on the two times $t$ and $t_{w}$, an action such as 
that in Eq. (58) (with an added $\tau$-dependent mass term) suggests that different time slices factorize in the effective theory for fluctuations. This picture is only approximate, in that for time ratios not far from one, the subleading terms neglected in Eq. (58) will generate corrections. Thus we expect that, for long times and large $t / t_{w}$ ratios, $m\left(t, t_{w}\right)$ should be some kind of average of one-time quantities associated with $t$ and $t_{w}$. This picture suggests that one should also analyze the correlation length data by plotting it against an average between $t$ and $t_{w}$, the appropriate one being a geometric average $t_{\text {ave }}=\sqrt{t t_{w}}$ [which corresponds to the simple average $\tau_{\text {ave }}=\left(\tau+\tau_{w}\right) / 2$ for the proper times, where $\left.\tau=\ln t\right]$.

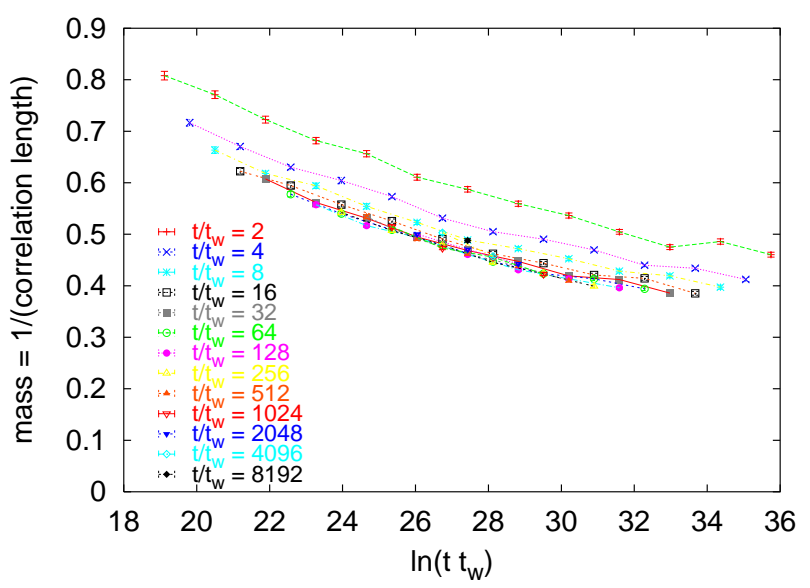

FIG. 31. Mass (inverse correlation length) as a function of the geometric average between $t$ and $t_{w}$. Each set of points corresponds to a fixed ratio $t / t_{w}$. The horizontal axis corresponds to $\ln \left(t t_{w}\right)$.

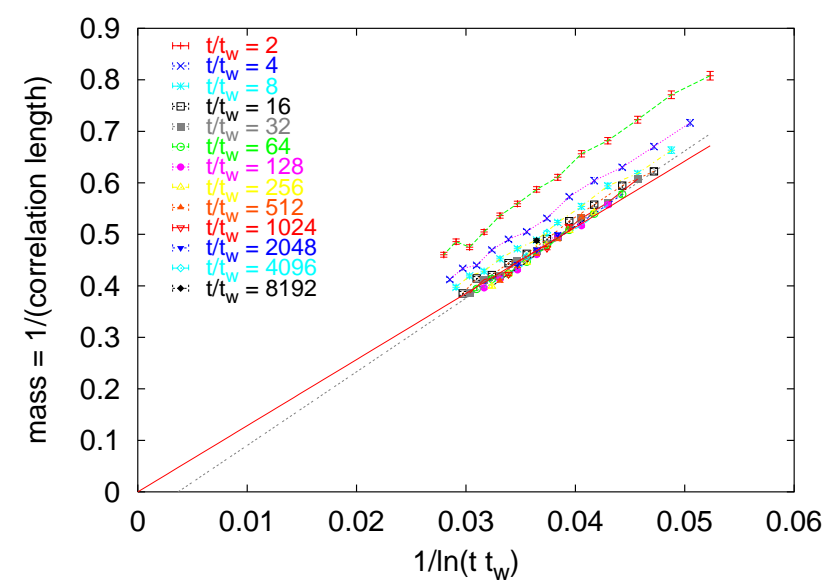

FIG. 32. Mass (inverse correlation length) as a function of the geometric average between $t$ and $t_{w}$. Each set of points corresponds to a fixed ratio $t / t_{w}$. The horizontal axis corresponds to $1 / \ln \left(t t_{w}\right)$.

With the above picture in mind, we now re-analyze the data in our simulations by replotting the mass $m\left(t, t_{w}\right)$ as a function of $t t_{w}$ in Fig. 31 .

As Fig. 31 shows, the curves for fixed $t / t_{w}$ ratio eventually converge to an asymptotic curve for large $t / t_{w}$. As discussed above, this is consistent with the subleading corrections in Eq. (58) becoming negligible for large $t / t_{w}$ ratios. In Figs. 32 and 33 we attempt to obtain more detailed information about the time dependence of the mass. In Fig. 32 we show fits of $m\left(t, t_{w}\right) \equiv 1 / \xi\left(t, t_{w}\right)$ to a $1 / \ln \left(t t_{w}\right)$ dependence. If a nonzero value is allowed for the mass at infinite time, the fitted value obtained is negative, meaning that the mass actually goes to zero at very large, but finite, times $\left(t t_{w} \sim 10^{276}\right)$. In practice, this fit is as good as a fit with the mass going to zero only at infinite time: in both cases $\chi^{2}$ per degree of freedom is of order $10^{-4}$.

In Fig. 33 we show the fit $m\left(t, t_{w}\right) \equiv 1 / \xi\left(t, t_{w}\right)$ to a power law $\left(t t_{w} / t_{0}^{2}\right)^{-a}$. If a finite mass is allowed at infinite times, an exponent $a \approx 0.10$ is obtained. If, instead, the value of the mass is assumed to be zero at infinite times, $a \approx 0.04$ is obtained. The $\chi^{2}$ per degree of freedom is again of the order of $10^{-4}$ for both fits.

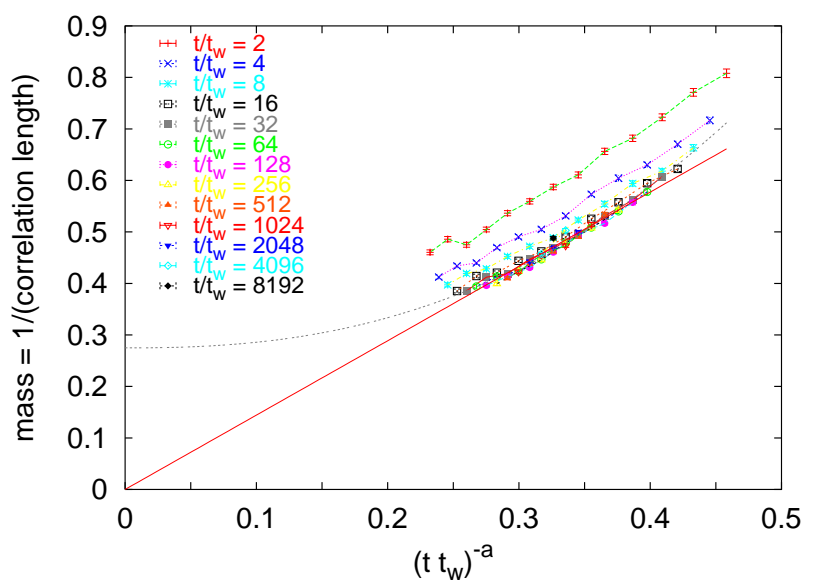

FIG. 33. Mass (inverse correlation length) as a function of the geometric average between $t$ and $t_{w}$. Each set of points corresponds to a fixed ratio $t / t_{w}$. The horizontal axis corresponds to $\left(t t_{w}\right)^{-a}$ with $a=0.04$.

In summary, the simulation results indicate that (i) $m\left(t, t_{w}\right)$ is asymptotically (for large $t, t_{w}$ and $t / t_{w}$ ) only a function of $t t_{w}$; (ii) $m\left(t, t_{w}\right)$ is a very slow monotonous decreasing function of $t t_{w}$, consistent both with the $m \approx$ $1 / \ln \left(t t_{w}\right)$ and with the $m \approx\left(t t_{w} / t_{0}^{2}\right)^{-a}$ functional forms; (iii) the values of the correlation length $\xi=1 / m$ are of the order of only a few lattice constants for the timescales accessible in the simulations; (iv) the extrapolation of the data fits is consistent with the expected $m \rightarrow 0$ behavior for long times, but a nonzero mass at infinite times cannot be excluded.

Previous studies [24,67] have found a correlation lengthscale $\xi\left(t_{w}\right)$ growing with $t_{w}$ that is in the range of 
3 to 4 lattice spacings for the waiting times and temperatures we have considered. Those authors determined the correlation length from the spatial decay of a four point correlation function [67]

$$
G\left(r, t_{w}\right)=\frac{1}{N} \sum_{i=1}^{N} \frac{1}{t_{w}} \sum_{\tau=t_{w}}^{2 t_{w}-1}\left[\left\langle C_{\vec{r}_{i}}^{a b}(\tau) C_{\vec{r}_{i}+\vec{r}}^{a b}(\tau)\right\rangle\right]
$$

with $C_{\vec{r}_{i}}^{a b}(\tau) \equiv s_{\vec{r}_{i}}^{a}(\tau) s_{\vec{r}_{i}}^{b}(\tau)$, involving thus two copies of the system $a$ and $b$, with identical exchanges, and an average over thermal histories and disorder. Note that this "2-point correlator" differs from ours in that (i) it is evaluated at equal times, (ii) there is a further average over the noise, and, more importantly, (iii) the two-point functions that one further correlates are evaluated between two copies of the system evolving independently. The latter property implies that this length can only be tested experimentally with indirect measurements [23,68]. Interesting enough, the length we define in this paper is, in principle, accessible directly with local experimental probes. Even if representing different quantities the values reached by the two correlation-lengths are very short and very similar.

\section{B. Interplay between the waiting-time, linear coarse-graining size and correlation-length}

In this section we discuss the role played by the waiting and total times, $t_{w}$ and $t$, the coarse-graining linear size, $2 M+1$, and the correlation length, $\xi\left(t, t_{w}\right)$, in our measurements. We assume that the size of the system, $L$, is the largest scale in the problem that has diverged at the outset of the discussion.

For a finite but very long waiting-time, $t_{w 1}$, and a time-window $\left[t_{w 1}, t_{1}\right]$ on which one wishes to study the fluctuating dynamics, the correlation length, $\xi\left(t_{1}, t_{w 1}\right)$ is finite but very long. Choosing a cubic coarse-graining volume $V_{1}$ with linear size $2 M_{1}+1<\xi\left(t_{1}, t_{w 1}\right)$ one then accommodates $\left[\xi\left(t_{1}, t_{w 1}\right) /\left(2 M_{1}+1\right)\right]^{d}$ cells within each correlated volume $\left[\xi\left(t_{1}, t_{w 1}\right)\right]^{d}$. According to the discussions in previous sections, the local coarse-grained two-time functions, defined on each cell, have different time-reparametrizations, $h_{i}(t)$, that vary smoothly in real space until reaching the correlation length, $\xi\left(t_{1}, t_{w 1}\right)$, when they completely decorrelate. By taking a large coarse-graining volume we ensure that the underlying effect of disorder is erased. This kills the fluctuations in the external function $f_{i}$, [see Eq. (16)] as well as erasing all fluctuations in $q_{\mathrm{EA}}^{i}$. The fluctuations in the local timereparametrizations give rise to a distribution of values of the local coarse-grained correlations, $\rho\left(C_{i}^{c g}\left(t_{1}, t_{w 1}\right)\right)$ that we study numerically in Sec. IV.

For another finite, but longer waiting-time, $t_{w 2}>t_{w 1}$, the zero-mode discussed in Sec. III becomes flatter and one expects the amount of fluctuations to increase. If we also enlarge the time-window $\left[t_{w_{2}}, t_{2}\right]$ to be analyzed in such a way that, say, the global correlation, $C$, remains unchanged, $C\left(t_{1}, t_{w 1}\right)=C\left(t_{2}, t_{w 2}\right)$, the new correlation length is longer than the previous one, $\xi\left(t_{2}, t_{w 2}\right)>$ $\xi\left(t_{1}, t_{w 1}\right)$ (see the results in Sec. V A). If we keep the same coarse-graining linear size as before, i.e. $2 M_{1}+1$, we expect the level of fluctuations in the reparametrizations to increase and the PDF $\rho\left(C_{i}^{c g}\left(t_{2}, t_{w 2}\right)\right)$ to be wider than $\rho\left(C_{i}^{c g}\left(t_{1}, t_{w 1}\right)\right)$. If, simultaneously to increasing the values of the times, we also increase the coarse-graining volume so as $(2 M+1)$ scales with $\xi\left(t, t_{w}\right)$, we should be able to maintain the amount of fluctuations. More precisely, the PDF computed with the new times, $\left(t_{2}, t_{w 2}\right)$, and the new coarse-graining size $2 M_{2}+1$, should be identical to the PDF computed with $\left(t_{1}, t_{w 1}\right)$ and $2 M_{1}+1$.

This procedure can be taken further to postulate that a scaling limit in which a stable distribution of fluctuations is reached. This limit is such that the waiting-time and the subsequent time go to infinity together keeping the global correlation fixed to a prescribed value $\mathrm{C}$ (note that this double limit can be more general than the case in which $t_{w}$ and $t$ are proportional), and the coarse-graining volume diverges together with the correlation length between $t$ and $t_{w}$, i.e. $\xi\left(t, t_{w}\right) / M$ is held fixed. If in this limit the distribution of local two-time functions remains non-trivial, then dynamical heterogeneous regions of all sizes exist.

Even though for a system with an asymptotic reparametrization invariant action the correlation length is expected to diverge asymptotically, in Sec. V A we showed that $\xi\left(t, t_{w}\right)$ is still very short for the times accessible numerically in the spin-glass model that we study here. Thus, one cannot study the dynamics using a coarse-graining volume such that $a \ll 2 M+1 \ll \xi\left(t, t_{w}\right)$ (with $a$ the lattice spacing).

Another different, but also interesting, regime in which to study fluctuations is the following. Let us take $t_{w}$ and $t$ very long but finite leading to a finite correlation length $\xi\left(t, t_{w}\right)$. If one takes the coarse-graining linear size to be of order $2 M+1 \sim \xi\left(t, t_{w}\right)$ each block leading to a point in the construction of the PDFs is, roughly speaking, an independent model with finite size $L=\xi\left(t, t_{w}\right)$. The results in Sec. VIII A suggest that the fluctuations in the global two-time functions of finite size systems have a very similar behavior to the local ones discussed above.

Finally, if we use a coarse-graining volume with linear size $2 M+1 \gg \xi\left(t, t_{w}\right)$, we do not expect to have any fluctuations and the PDFs should be delta peaks on single values.

\section{SCALING OF LOCAL SUSCEPTIBILITY}

In this section we study the dynamic behavior of the local integrated responses. First we select the magnitude of the applied perturbation by studying the behavior of 
the global susceptibility. Next we study the evolution of the probability distributions of coarse-grained and noiseaveraged quantities.

\section{A. Choice of field strength}
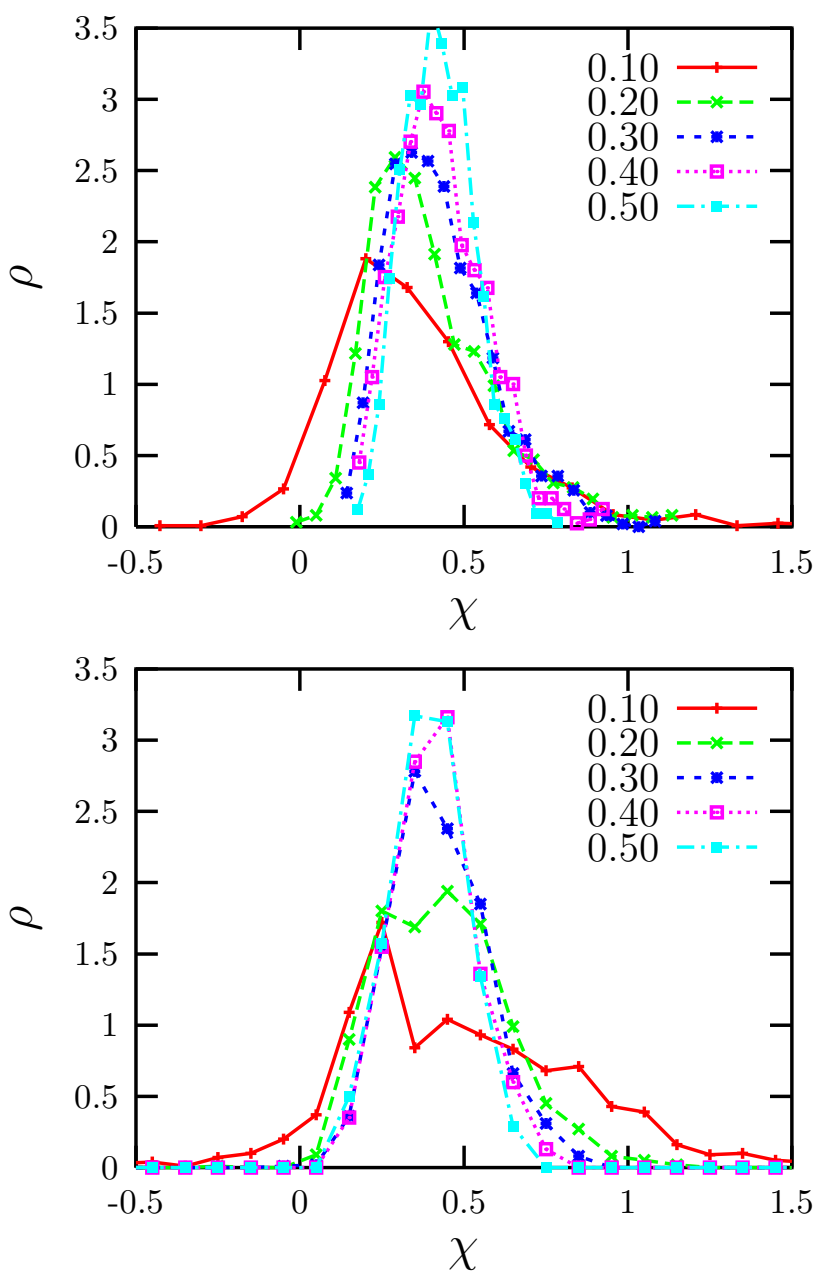

FIG. 34. Distribution of global staggered susceptibilities for small 3 EA models. The field is applied at $t_{w}=10^{4}$ MCs and held fixed subsequently. The integrated response is measured at $t=4 \times 10^{4} \mathrm{MCs}$. In the first panel we used one noise and $10^{3}$ field realizations; in the second panel we used one random field and $10^{3}$ noise realizations. The curves correspond to different field strengths given in the key. $L=8, T=0.7$ (noise averaged) and $T=0.8$ (field-averaged).

We choose the strength of the perturbing field, $h$, in such a way that (i) there are no negative global susceptibilities; (ii) there are no global susceptibilities with a magnitude larger than the value allowed by FDT $1 / T$; (iii) we select the range of field strengths such that linear response holds, i.e. we find the maximum strength for which the distribution is stable. The first two conditions yield a lower bound on $h, h_{\mathrm{MIN}}$, the third determines its maximum possible value, $h_{\mathrm{MAx}}$.

In Fig. 34 we show the outcome of these tests. We plot the PDF of the global staggered susceptibilities where we used two different procedures to draw the histograms. In the first plot, we used $10^{3}$ realizations of a random field, $\eta_{i}=\eta \epsilon_{i}$ with $\epsilon_{i}= \pm 1$ with probability $\frac{1}{2}$, and only one thermal noise (note that perturbed and unperturbed copies are evolved with the same thermal noise). We then draw the distribution of global values,

$$
\chi\left(t, t_{w}\right) \equiv \frac{1}{\eta} \sum_{i=1}^{N}\left[s_{i}^{h}(t)-s_{i}^{0}(t)\right] \epsilon_{i}^{k},
$$

with one point per field realization, $k=1, \ldots \#_{\text {fields }}$. In the second case, we used only one random field realization and we draw the distribution of global values,

$$
\chi\left(t, t_{w}\right) \equiv \frac{1}{\eta} \sum_{i=1}^{N}\left[s_{i k}^{h}(t)-s_{i k}^{0}(t)\right] \epsilon_{i},
$$

with one point per thermal noise realization, with $k=$ $1, \ldots, \#_{n o i s e s}$. We see that for such a small system size the probability distributions $\rho(\chi)$ change quite a bit with the strength of the applied field (even if the average and variance are quite stable, see Fig. 36 below). For larger system sizes, $L=16$, see Fig. 35, the probability distribution stabilizes for $\eta \geq \eta_{\mathrm{MIN}} \sim 0.2$.

The plot in Fig. 36 shows the average and the variance of the probability distribution for systems of size $L=8$ and $L=16$ with $10^{3}$ noise realizations, and $L=8$ with $10^{3}$ field realizations. We confirm that $\eta_{\mathrm{MIN}}=0.2$ and we see that the behavior starts being non-linear at around $\eta_{\text {MAX }} \sim 0.7$.

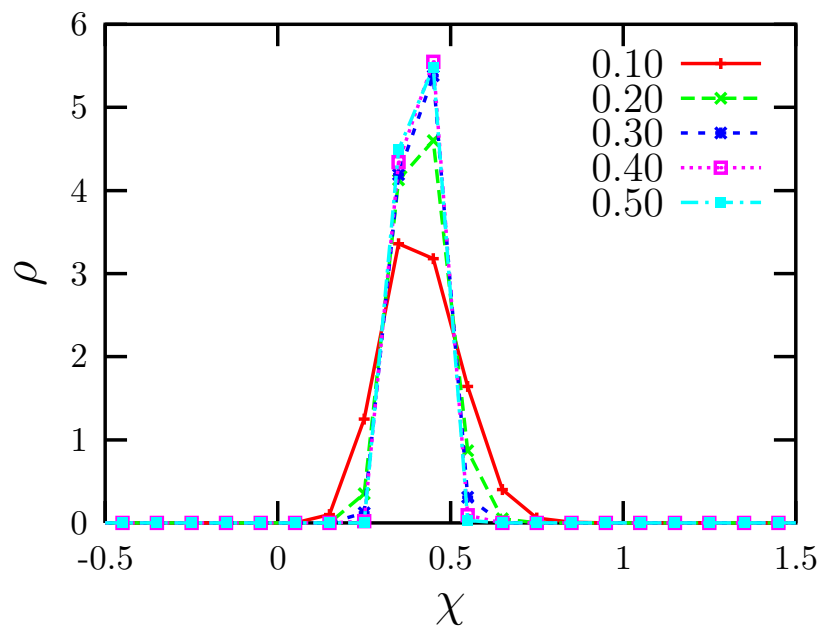

FIG. 35. Distribution of the global staggered susceptibilities for a $3 d$ EA model with linear size $L=16$ at $T=0.7$ using $10^{3}$ noise realizations and one perturbing field with strength given in the key. $t_{w}=10^{4} \mathrm{MCs}, t=4 \times 10^{4} \mathrm{MCs}$ and $\tau_{t}=t / 10$ MCs. 


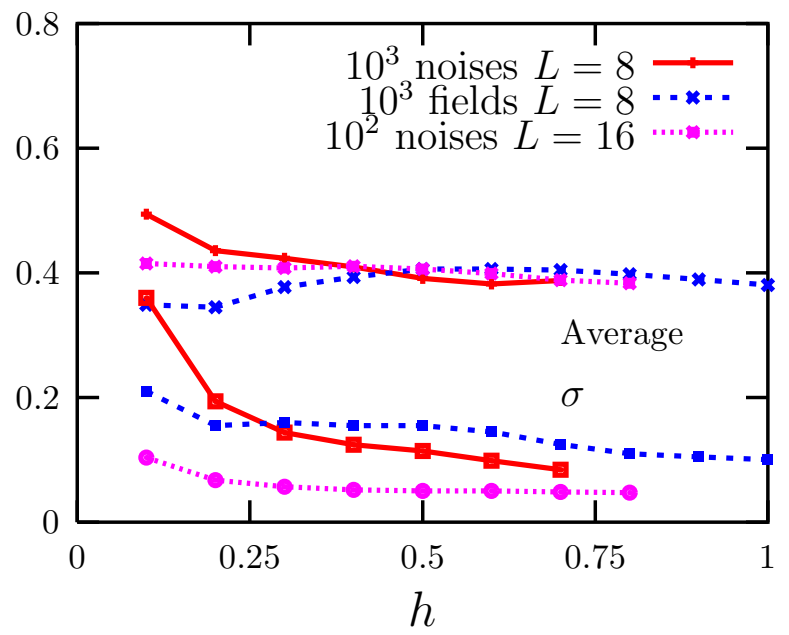

FIG. 36. Average and variance of the distribution of global staggered susceptibilities in the $3 d$ EA model against the field strength. The parameters are as in Figs. 34 and 35.

We see that field strengths between 0.2 and 0.7 comply with our criteria. In what follows we use $\eta=0.25$ and we focus on the noise-averaged and coarse-grained definitions of the local susceptibilities given in Eqs. (6) and (8) eventually averaged over many field realizations.

\section{B. Time-dependent distribution functions}

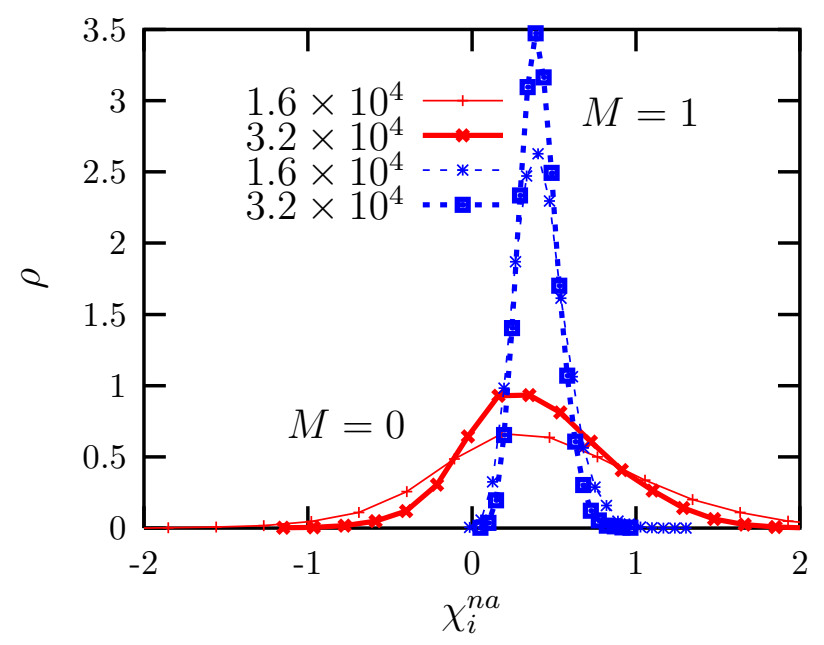

FIG. 37. Check of $t / t_{w}$ scaling of the distribution of noise-averaged local susceptibilities with no coarse-graining, $M=0$ and with coarse-graining, $M=1$. The ratio is $t / t_{w}=2$. When $t_{w}=1.6 \times 10^{4}$ MCs we used 1200 noise realizations. When $t_{w}=3.2 \times 10^{4}$ MCs we used 200 noise realisations.

Here we study the evolution of the PDF of local susceptibilities computed as in Eqs. (6) and (8). The figure shows that the $t / t_{w}$ scaling is worse for the susceptibility than it is for the correlations.

\section{Coarse-grained data}

In Figs. 38-40 we show the PDF of local susceptibilities coarse-grained over different volumes. These figures are the counterpart of Figs. 15-17 where we showed the coarse-grained data for the local correlations.

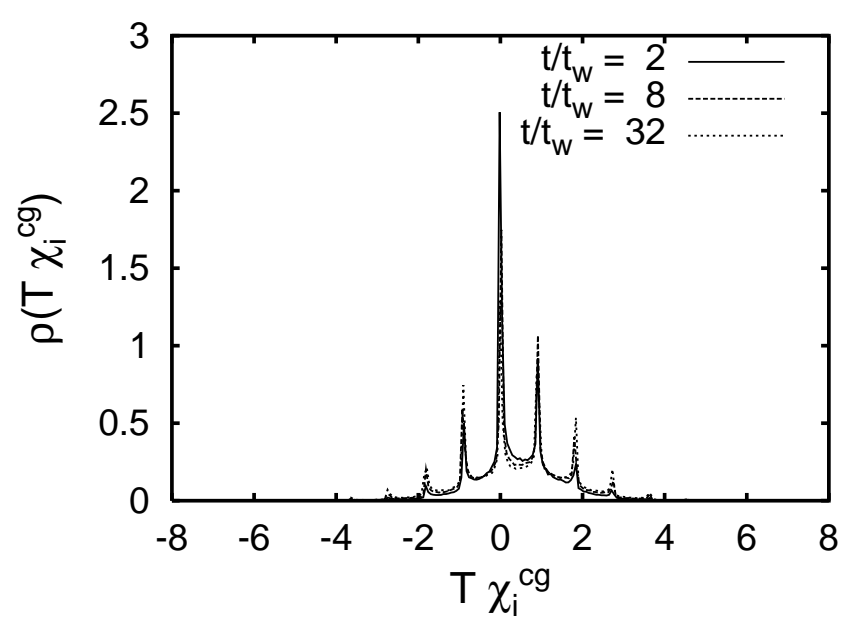

FIG. 38. Distribution of local coarse-grained staggered susceptibilities. $L=32, T=0.8$ and $\eta=0.25$. The waiting times are $t_{w}=4 \times 10^{4} \mathrm{MCs}, 1.6 \times 10^{4} \mathrm{MCs}$, and $6.4 \times 10^{4}$ MCs. Three ratios are considered, $t / t_{w}=2,8,32$. The coarse-graining volume is $V=2 M+1=1$.

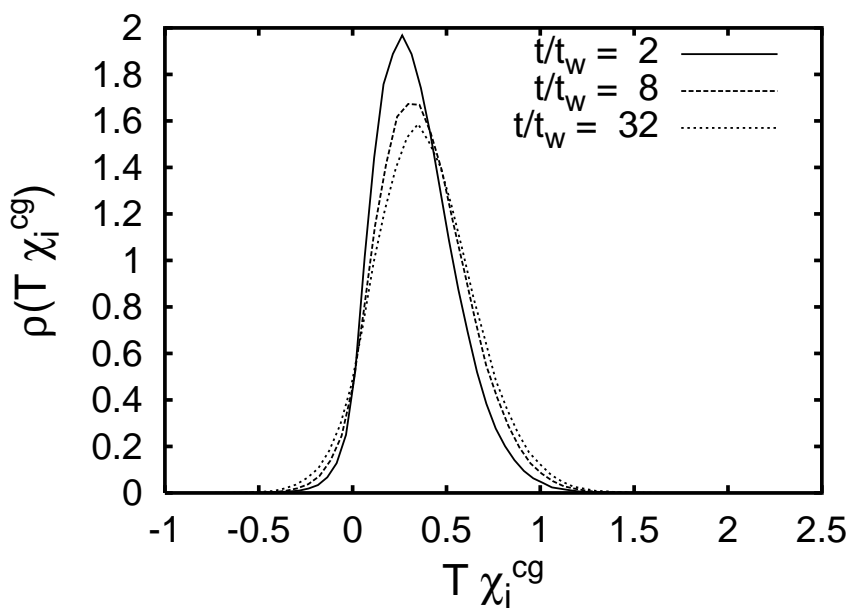

FIG. 39. Distribution of local coarse-grained staggered susceptibilities. Same data as in Fig. 38 with $M=1$.

In Fig. $382 M+1=1$, in Fig. $392 M+1=3$, and in Fig. $402 M+1=13$. The strictly local susceptibilities 
shown in Fig. 38 are distributed almost in a discrete way, one sees peaks at precise values of $\chi_{i}^{c g}$ that extend also to the the negative side of the axis. When $M=1$, the negative suseptibilities have not yet disappeared and the positive tail goes also beyond $T \chi_{i}^{c g}=1$, see Fig. 39 . Finally, when $M=6$ the PDFs are much narrower with no support on negative values nor values that go beyond $T \chi_{i}^{c g}=1$. Note, however, that the linear size of this coarse-graining volume, $2 M+1=13$, is larger than the maximum correlation length reached with these times, say $\xi\left(t, t_{w}\right) \sim 5$ (see Sec. V).

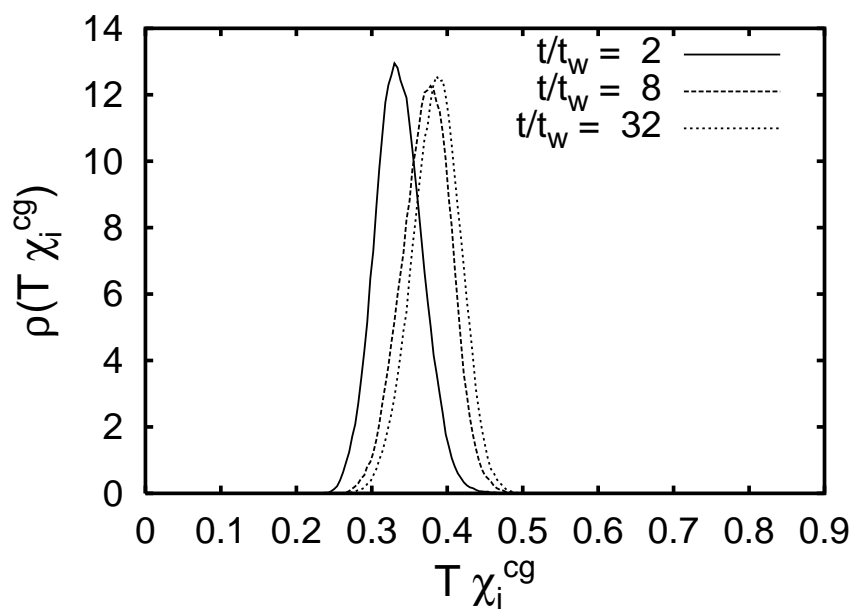

FIG. 40. Distribution of local coarse-grained staggered susceptibilities. Same data as in Fig. 38 with $M=6$.

\section{THE LOCAL FLUCTUATION-DISSIPATION RELATION}

Which of the possibilities listed in Sec. IIF does the local FDR follow? The aim of this section is to show numerical evidence for the following statements:

(i) The local coarse-grained two-time correlations and responses are constrained to follow the global curve $\tilde{\chi}(C)$ : the dispersion along the $\tilde{\chi}(C)$ curve is much more important than the dispersion perpendicular to this curve. For fixed $t_{w}$ and increasing values of $t$ the dispersion along the $\tilde{\chi}(C)$ curve increases. This is as predicted by the sigma-model argument of Sec. III. Equation (33) holds for these functions.

(ii) The local noise-averaged correlations do not necessarily follow the global curve. Coarse-graining this data does not lead to concentrating the distribution of $\left(C_{i}, T \chi_{i}\right)$ pairs around the global curve. Equation (33) does not hold. It is hard to test numerically if Eq. (31) holds since there is no constraint on the location of the pairs in the $C-T \chi$ plane.

(iii) The mesoscopic fluctuations (with no average over the noise) behave as in (i).
We provide numerical evidence for these statements by plotting the joint probability distribution of pairs $\left(C_{i}, T \chi_{i}\right)$ evaluated at a pair of times $\left(t_{w}, t\right)$, and its projection on the $2 d$ plane $C-T \chi$.

\section{A. Coarse-grained two-time functions}

In Fig. 41 we plot the projection of the joint probability distribution of coarse-grained two-time functions $\left(C_{i}^{c g}, T \chi_{i}^{c g}\right)$ on the $C-T \chi$ plane. The data correspond to a system of linear size $L=64$ at $T=0.8$. The coarsegraining linear size is $2 M+1=3$ that is of the order of the correlation length $\xi\left(t, t_{w}\right)$. We study simultaneously three ratios of times, $t / t_{w}=2,8,32$, for $t_{w}=10^{4} \mathrm{MCs}$ in all cases. The contour levels are such that they include $66 \%$ of the weight of the joint PDF and they correspond to $t / t_{w}=2,8,32$ from inside to outside. Reasonably, as the times get more separated the values of the local correlations vary more and the region incircled by the contour level is wider. This plot shows that these distributions are very wide, as also indicated by Figs. 16 and 40 where we show $\rho\left(C_{i}\right)$ and $\rho\left(\chi_{i}\right)$, independently. Even though the contour levels are also wide in the $T \chi$ direction, they tilt in the horizontal direction of $C$, along the curve made by the crosses, that corresponds to $T \tilde{\chi}(C)$ for this $t_{w}$ and 10 values of $t$.

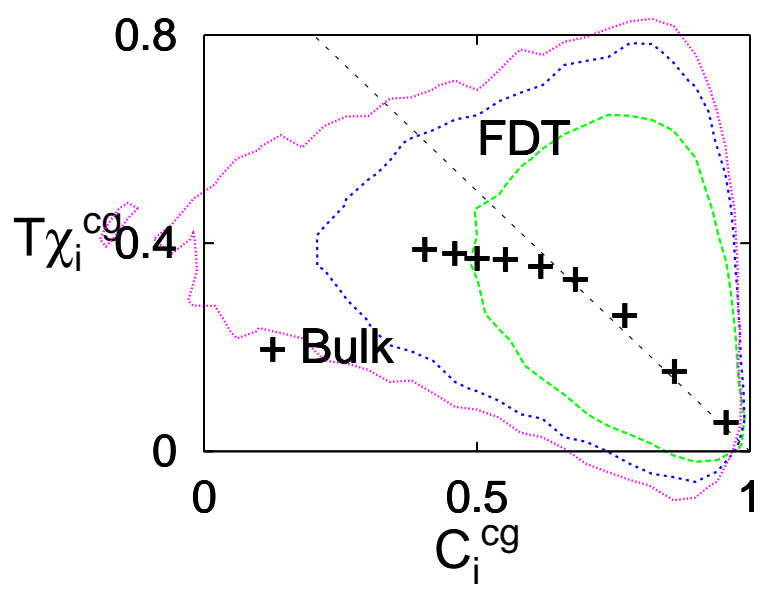

FIG. 41. Projection of the joint probability distribution of the coarse-grained local susceptibilities and correlations. $L=32, T=0.8$ and $M=1$ leading to $2 M+1 \sim \xi\left(t, t_{w}\right)$. The contour levels surround $66 \%$ of the weight of the distribution and each of them is drawn using a different ratio between the two times. From inside to outside $t / t_{w}=2,8,32$.

In Fig. 42 we see the effect of further coarse-graining the data corresponding to the same $t_{w}$ and $t$ as in Figs. 41. In the projected plot we see how the size of the cloud around the $T \tilde{\chi}(C)$ plot is reduced. The longitudinal fluctuations that correspond to fluctuations in the 
function $f_{i}$ that characterizes the local correlations are killed very quickly by the coarse-graining (see the theoretical background for this in Sec. III and the related effect in the values of the local correlation in Sec. IV F). The transverse fluctuations are also reduced but in a weaker manner. These are related to the fluctuations in the local reparametrizations and, as we argued in Secs. III and IV F, they should survive in the limit of long times and large coarse-graining volumes. See Sec. VIII for similar results for the joint PDFs of the global quantities computed using sytems of small size.

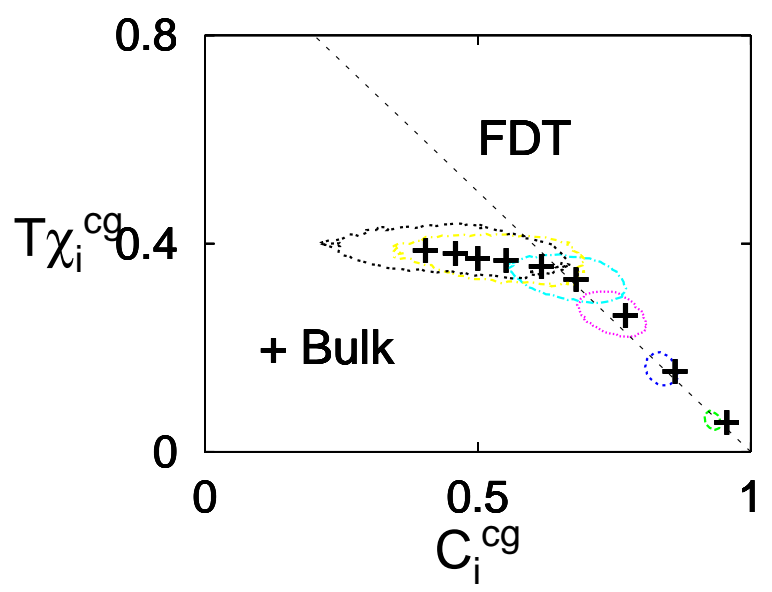

FIG. 42. Projection of the joint probability distribution of the coarse-grained local susceptibilities and correlations. $L=32, T=0.8$ and $M=6$. Note that here $2 M+1 \gg \xi\left(t, t_{w}\right)$. The contour levels surround $66 \%$ of the weight of the distribution and each of them is drawn using a different ratio between the two times with $t / t_{w}$ increasing from right to left.

\section{B. Noise-averaged two-time functions}

In Fig. 43 we show the joint probability distribution of the noise-averaged local correlations and susceptibilities. We see that the distribution is concentrated about the FDT line and does not bend in the direction of the nontrivial part of the global $\tilde{\chi}(C)$ as the coarse-grained data does, see Fig. 41. This indicates that the disorder induced fluctuations are not controlled by the sigma model argument of Sec. III. Note that with no coarse-graining the Edwards-Anderson parameter fluctuates from site-tosite and hence the value of the correlation at which the sites enter the slow scale varies. This result resembles Fig. 3 or Fig. 4 only that the extent of the fluctuations in $C_{i}^{n a}$ is narrower than in these sketches.

In Sec. VIII we shall observe a very similar behavior of the noise-averaged mesoscopic fluctuations in finite size SK models.

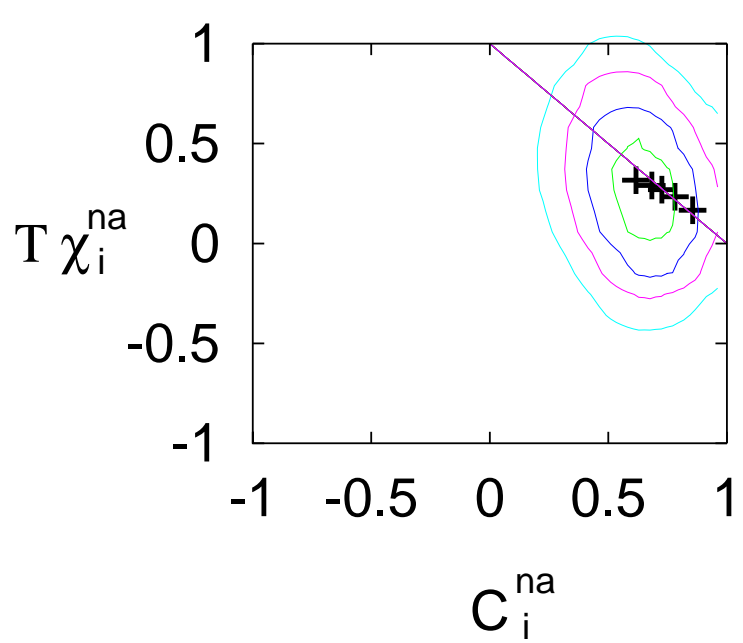

FIG. 43. The projection of the joint PDF on the $C-T \chi$ plane, noise averaged quantities, $L=32, t_{w}=1.6 \times 10^{4}$ MCs and $t=4.8 \times 10^{4}$ MCs. The FDT prediction is represented with a straight line.

\section{Effect of coarse-graining on already noise-averaged data}

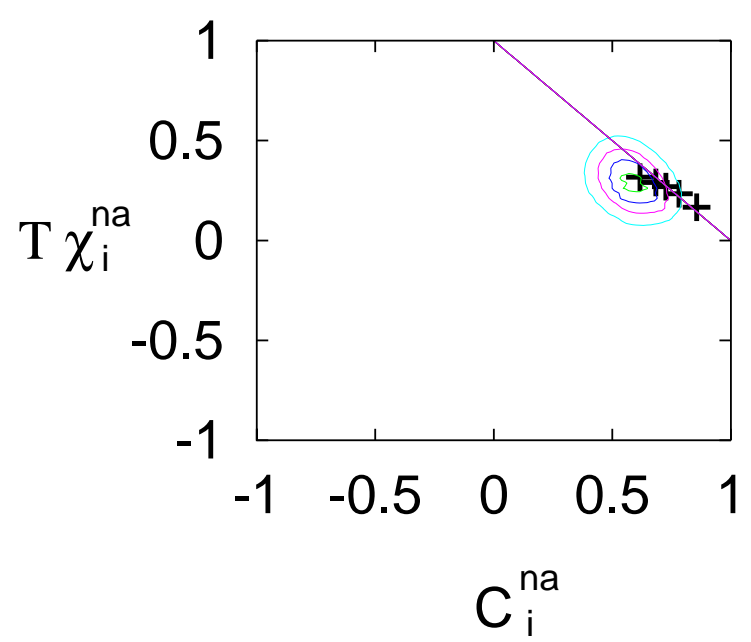

FIG. 44. Same as in the previous figure, the coarse-graining volume has with linear size $M=1$.

In Figs. 44 and 45 we test the effect of coarse-graining on the joint probability distribution of noise-averaged local correlations and susceptibilities. In Fig. 44 the linear size of the coarse-graining box is $2 M+1=3$ while in Fig. 45 it is $2 M+1=7$. The system size is $L=32$ and $T=0.7$. We see that in all cases the distribution follows the FDT line. Indeed, averaging over the noise kills all thermal fluctuations and hence do not allow for the fluctuations in the time-reparametrization that cause the 
bending of the data along the $\tilde{\chi}(C)$ curve. This is consistent with the results in Fig. 22 where we checked the effect of coarse-graining the already noise-averaged local correlations.

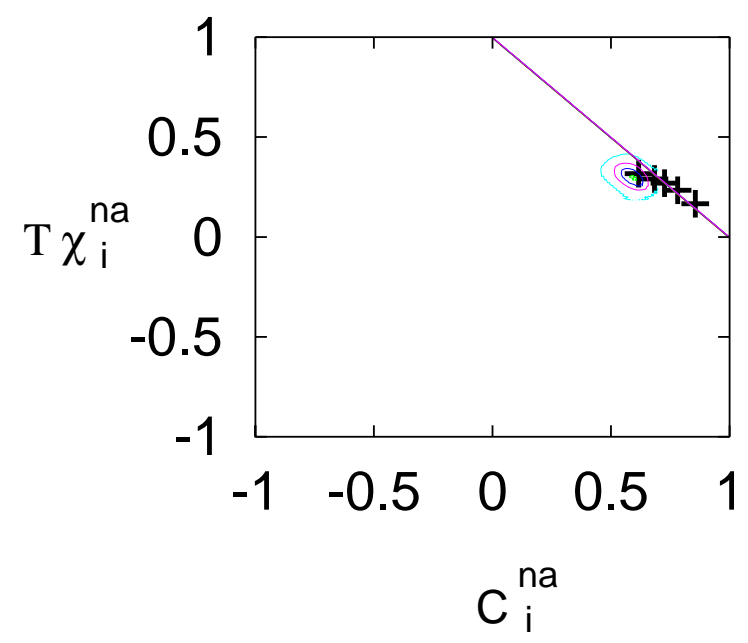

FIG. 45. Same as in the previous two figure, the coarse-graining has linear size $M=3$.

\section{FINITE SIZE SYSTEMS}

A. Barrat and Berthier [69] showed that the modification of the FDT in a $3 d$ EA model of finite size that is evolving out of equilibrium (that is to say for $t$ and $t_{w}$ that are well below the finite equilibration time $t_{\mathrm{EQ}}$ ), has a very similar behavior to the one found in the thermodynamic limit. The system will eventually equilibrate but, as long as one keeps the times to be shorter than $t_{\mathrm{EQ}}$, the global dynamics is very much the typical one of an out of equilibrium system.

In this section we study the time-dependence of the distributions of the global correlation and susceptibility for finite-size samples of both finite- $d$ and infinite- $d$ spinglasses. We show that the "mesoscopic" fluctuations, i.e. the fluctuations of the global quantities due to finite size effects, behave very similarly to the local coarse-grained ones in finite $d$. Moreover, we test the relation between the fluctuations in the susceptibility and the correlation and we find that they are also constrained to follow the global parametric $\tilde{\chi}(C)$.

\section{A. The $3 d$ EA model}

In Figs. 46 and 47 we show the evolution of the distributions of the global correlation, $C\left(t, t_{w}\right)$, and integrated response, $\chi\left(t, t_{w}\right)$, with time $t$ and for fixed $t_{w}$, respectively. We construct the distribution functions using one data point for the global correlation and linear integrated response of the full system obtained using one noise realization. The details of the system are given in the caption and key. We use $L=8$ so as to be able to access smaller values of the global correlation than is possible in larger size systems. This small system size also allows us to have PDFs that are rather wide.

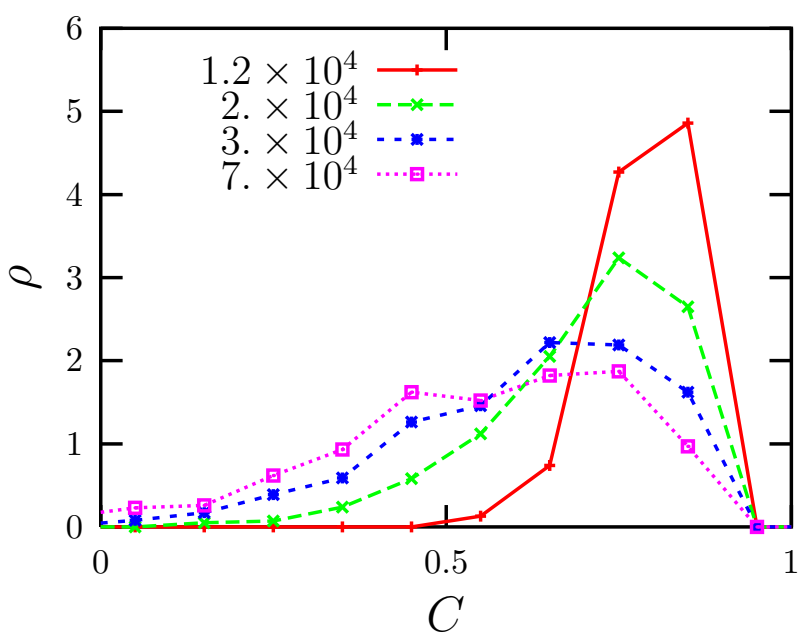

FIG. 46. Evolution of the PDF of the global correlation for a system with linear size $L=8$ at $T=0.7$. The waiting-time is $t_{w}=10^{4}$ MCs and the different values of the subsequent time $t$ used in each curve are given in the key.

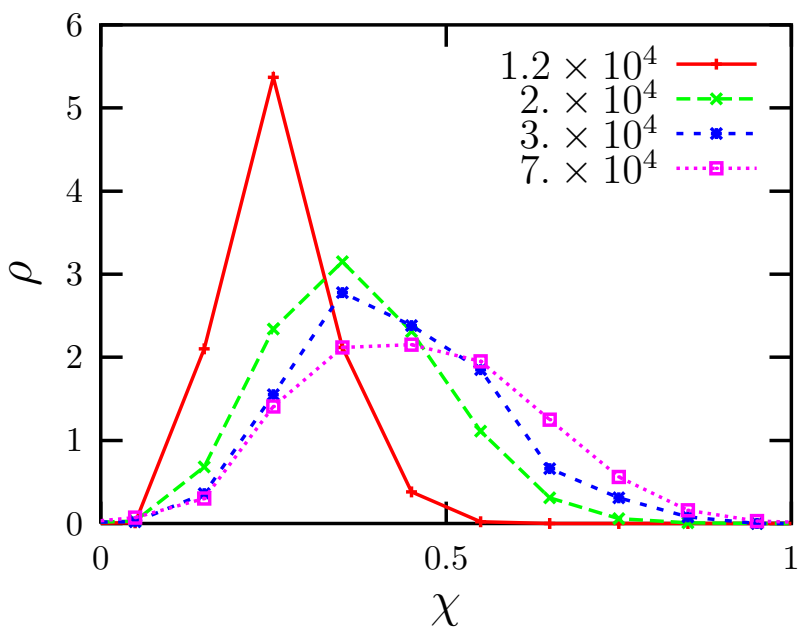

FIG. 47. Evolution of the PDF of the global susceptibility for the same system as above. $\eta=0.3$.

In Figs. 46 and 47 we display the evolution in time of the PDFs for the global correlation and the global suseptibility of a system with $L=8$. We see that the distributions get wider as the separation between the times increases. In Fig. 48 we correlate the fluctuations in the global correlation and suseptibility. We see that, as in the study of the local coarse-grained quantities in a larger 
system, the contour levels of the joint PDF are tilted in the direction of the global $\tilde{\chi}(C)$ that is indicated with crosses.

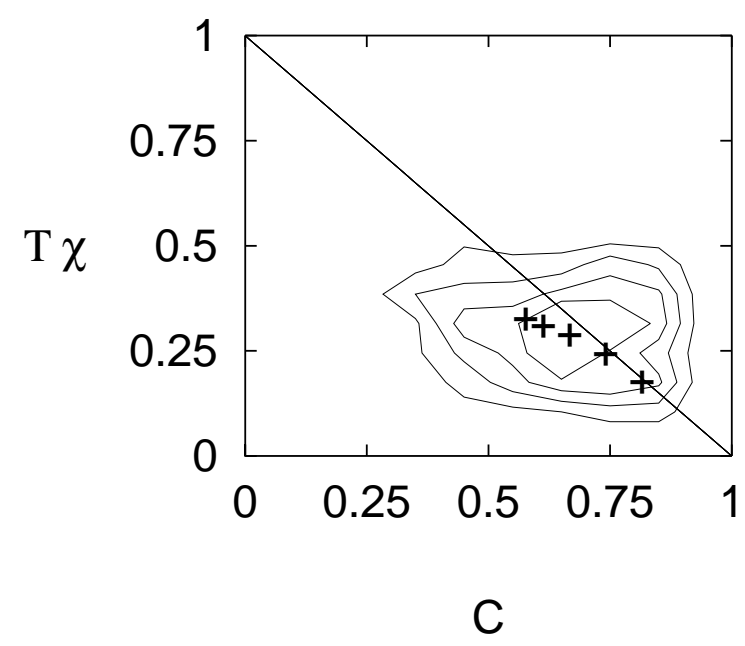

FIG. 48. Projection of the joint probability distribution of the global correlations and integrated responses for a $3 d \mathrm{EA}$ model with linear size $L=8$ at $T=0.7$. The strength of the applied field is $\eta=0.25$. The contour levels correspond to the joint PDF for the waiting-time $t_{w}=10^{4} \mathrm{MCs}$ and $t / t_{w}=4$. One realization of the random exchanges and $10^{3}$ thermal histories are used to construct the PDF. $\tau_{t}=t / 10 \mathrm{MCs}$. The straight line indicates the FDT prediction and the crosses indicate the time evolution of the average $\chi(C)$.

\section{B. The SK model}

With the aim of giving additional support to our claim that noise-averaged and coarse-grained dynamic fluctuations behave differently we analysed the dynamic fluctuations in the fully connected SK model on a finite size lattice.

The SK model is defined in Eq. (2), where the couplings $J_{i j}$ connect all sites and are chosen randomly from a bimodal distribution. An infinite size system undergoes a thermodynamic and dynamic phase transition at $T_{c}=1$. When $N \rightarrow \infty$ the dynamics in the low temperature phase was solved analytically in the asymptotic limit of long-times (although finite with respect to the size of the system) [7]. The solution has a rather peculiar structure with the relaxation taking place in a sequence of hierarchically organized correlation scales. These are intimately related to the FDR, $\tilde{\chi}(C)$, that takes a curved form for values of the correlation that fall below the EdwardsAnderson order parameter.

Several studies of the growth of the equilibration time with the size of the system, $N$, indicate that this increases approximately as $t_{\mathrm{EQ}} \propto e^{c N^{\alpha(T)}}$ [70] with $c$ a numerical constant and the exponent $\alpha(T)$ increasing from
$1 / 3$ at $T_{c}$ to 0.5 at $T=0.4 T_{c}$ (see also Ref. [71]). Even though these studies used a Gaussian distribution of couplings we take these results as an indication that even for small samples, e.g. $N=512$, we have a very large timewindow with nonequilibrium effects before equilibration takes place. (We chose to use $N=512$ just to have the same number of spins as for the $3 d$ EA with $L=8$ used in Sec. VIII A.)

Numerical studies have found results in agreement with the analytic prediction of there being a sequence of global correlation scales and a curve FDR out of equilibrium [72]. Exactly how the scaling laws and the $\tilde{\chi}(C)$ prediction are modified due to finite size effects has not been carefully investigated.

In this section we present results from a numerical simulation of the SK model with bimodal interactions using MC dynamics with the heat bath algorithm at $T=0.4$ [73]. We pay special attention to the fluctuations induced by the finite size of the systems.

\section{Finite size fluctuations of global quantities}

The natural counterpart to the coarse-grained local correlations and responses in finite dimensional models is, for a fully connected model, the global quantity itself. The latter fluctuates if the fully-connected system has a finite size.

In Fig. 49 we display the joint PDF for the global susceptibility and global correlation. We used $t_{w}=64$ MCs and we evolved the systems until $t=1024$ MCs. We constructed the distribution functions using $10^{5}$ pairs $\left(C\left(t, t_{w}\right), \chi\left(t, t_{w}\right)\right)$ calculated as follows. First we chose the value of the total time $t$ with which to calculate the global quantities, e.g. $t=128$ MCs. For a fixed realization of the random exchanges we used $10^{4}$ noise histories and thus obtained $10^{4}$ points. We repeated this procedure with 10 different realizations of disorder completing the set of $10^{5}$ data points. With this data we obtained a probability distribution. The crosses represent the average over the $10^{5}$ points for $t=65,70,128,256,512$, and 1024 MCs. In Fig. 49 we show the projection on the $C-T \chi$ plane of: three contour levels at $95 \%, 90 \%$, and $82 \%$ from the maximum of the joint PDF calculated for $t_{w}=64 \mathrm{MCs}$ and $t=1024 \mathrm{MCs}$ [panel (a)]; one contour level at $90 \%$ of the maximum of the joint PDF for four values of the total time, $t=128,256,512$, and 1024 MCs [panel (b)]. The straight represents the expected equilibrium curve.

We see that, similarly to the local coarse-grained fluctuations for the $3 d$ EA model, the distribution follows the global $T \tilde{\chi}(C)$ curve. The contour levels are inclined in the direction of the global curve. Thus, despite having very different time-scalings, these two models have very similar parametric joint PDF distributions. 

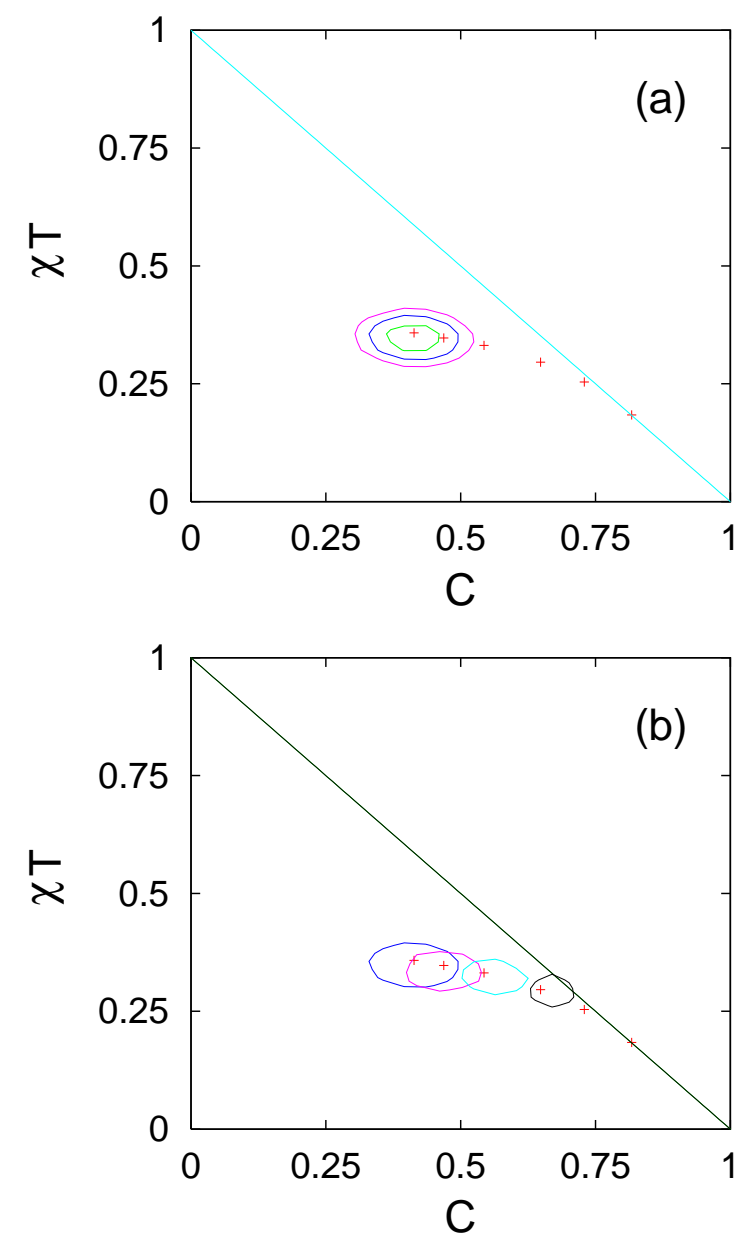

FIG. 49. Projection of the joint PDF for the global susceptibility and correlations of the sK model with $N=512$ and $\beta=2.5$. The strength of the applied field is $\eta=0.25$. The coarse-graining over time is done using $\tau=2$ for $t_{w}=64 \mathrm{MCs}$ and $t=65,70 \mathrm{MCs}$, and $\tau_{t}=2,4,8$, and $16 \mathrm{MCs}$ for $t=128$, 256,512 ,and 1024 MCs. The crosses indicate values averaged over the distribution, the straight line is the prediction from the FDT. In panel a) the contour levels are chosen at heights corresponding to $95 \%, 90 \%$, and $82 \%$ of the maximum in the PDF for the global correlations evaluated at $t_{w}=64 \mathrm{MCs}$ and $t=1024$ MCs. In panel b) the contour levels are at $90 \%$ of the maximum and they correspond to the PDFs calculated at $t_{w}=64 \mathrm{MCs}$ and $t=128,256,512,1024 \mathrm{MCs}$ from right to left.

\section{Fluctuations in the noise averaged local quantities}

In this section we follow a similar path to the one described in Sec. VII B for the $3 d$ EA model. We simulated a sK model with $N=512$ spins at $\beta=2.5$. The waitingtime chosen was $t_{w}=64$ MCs. We averaged the spinspin "local" self-correlation and integrated self-response for chosen pairs of times $t, t_{w}$ with $t=128,256,512$, and 1024 MCs over 1600 different noise realizations. With one realization of the random exchanges we thus obtained $N$ data points. To improve the statistics we repeated this procedure using 150 different sK models of the same size, i.e. with different realizations of the coupling strengths. Thus, the PDFs are constructed with 76800 datapoints as is shown in Fig. 50 (the crosses are the averaged values). The qualitative form of the distribution is very different from the one in Fig. 49. The orientation of the contour levels does not follow the $T \tilde{\chi}(C)$ curve but, instead, it is approximately parallel to the FDT straight line.
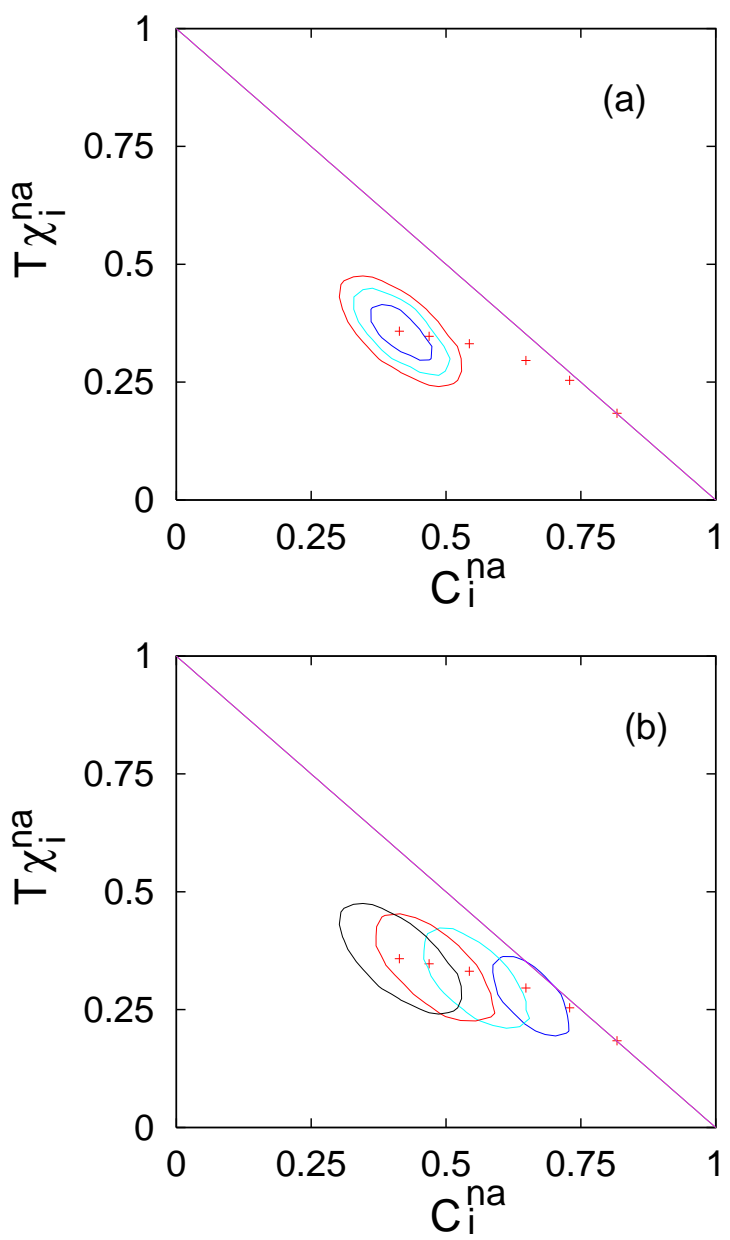

FIG. 50. Projection of the joint PDF for the noise-averaged "local" susceptibilities and correlations of the SK model with $N=512$ and $\beta=2.5$. The strength of the applied field is $\eta=0.125$. The coarse-graining times $\tau$ are chosen as in Fig. 49. The crosses indicate values averaged over the distribution, the straight line is the prediction from the FDT. In panel a) the contour levels are chosen at heights corresponding to $90 \%, 85 \%, 80 \%$ and they correspond to times $t_{w}=64$ MCs and $t=1024$ MCs. In panel $\mathrm{b}$ ) the contour levels are at $80 \%$ and they correspond to the joint PDF at $t_{w}=64 \mathrm{MCs}$ and $t=1024$ MCs.

These results are similar to the ones displayed in Sec. VII B for the joint PDF of the local noise-avareged correlations and integrated responses in the $3 d$ EA model. 


\section{Effect of partial noise averaging}

Finally, we studied the effect of partial averaging over the noise the global two-time functions in a still smaller system. By this we mean that we averaged over $10^{2}$ realizations of the thermal history the global correlation and integrated response of each of $6 \times 10^{4} \mathrm{SK}$ models with $N=128$ spins. Thus, we constructed the joint PDF with $6 \times 10^{4}$ points. The result is displayed in Fig. 51 . We see that averaging over the noise we destroy the behavior in Fig. 49: the contour levels are tilted toward the direction of the FDT line. These results approach the ones in Fig. 50.

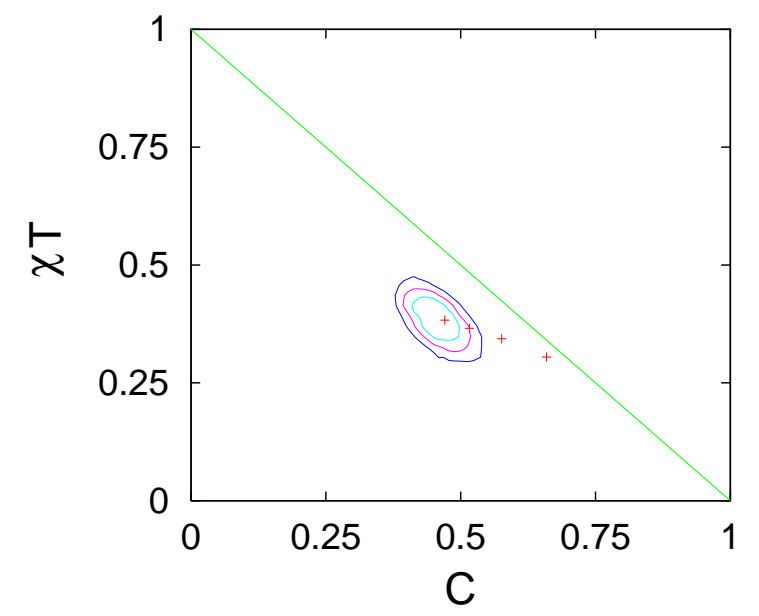

FIG. 51. Projection of the joint PDF for the global susceptibility and correlations of the sK model with $N=128$, $\beta=2.5$ and $\eta=0.1$. The global two-point functions for each system have been averaged over $10^{2}$ noise realizations. The coarse-graining times $\tau$ are chosen as in Fig. 49. The crosses indicate values averaged over the distribution, the straight line is the prediction from the FDT and the contour levels are chosen at heights corresponding to $80 \%, 70 \%$ and $60 \%$ of the maximum in the PDF evaluated at $t_{w}=64 \mathrm{MCs}$ and $t=1024$ MCs.

\section{GEOMETRIC PROPERTIES}

We have analyzed the local correlations and responses in terms of their PDFs and by looking directly at plots for their spatial fluctuations along a plane or line of spins. An alternate way to extract information about the spatial structure in spin glasses is through an analysis of geometric properties, such as the fractal properties of clusters of spins.

The clusters of spins that we have chosen to study are defined in a way that we believe makes close contact with possible experiments with local probes. The usual definition of a cluster in a spin glass is in terms of spins belonging to different ground states - since we have not calculated the ground states in our simulations, such a definition is clearly not available to us. The definition we have chosen for clusters is as follows: for a particular correlation $\mathrm{C}$, the cluster consists of all connected spins with correlation $C_{i}$ in the interval $[\mathrm{C}, \mathrm{C}+d \mathrm{C}]$ for a specified $d$ C. This definition is used for both coarse-grained and noise-averaged correlators. A similar approach could be easily implemented to analyze experimental data on local noise in mesoscopic regions in supercooled liquids and glasses [27-30].
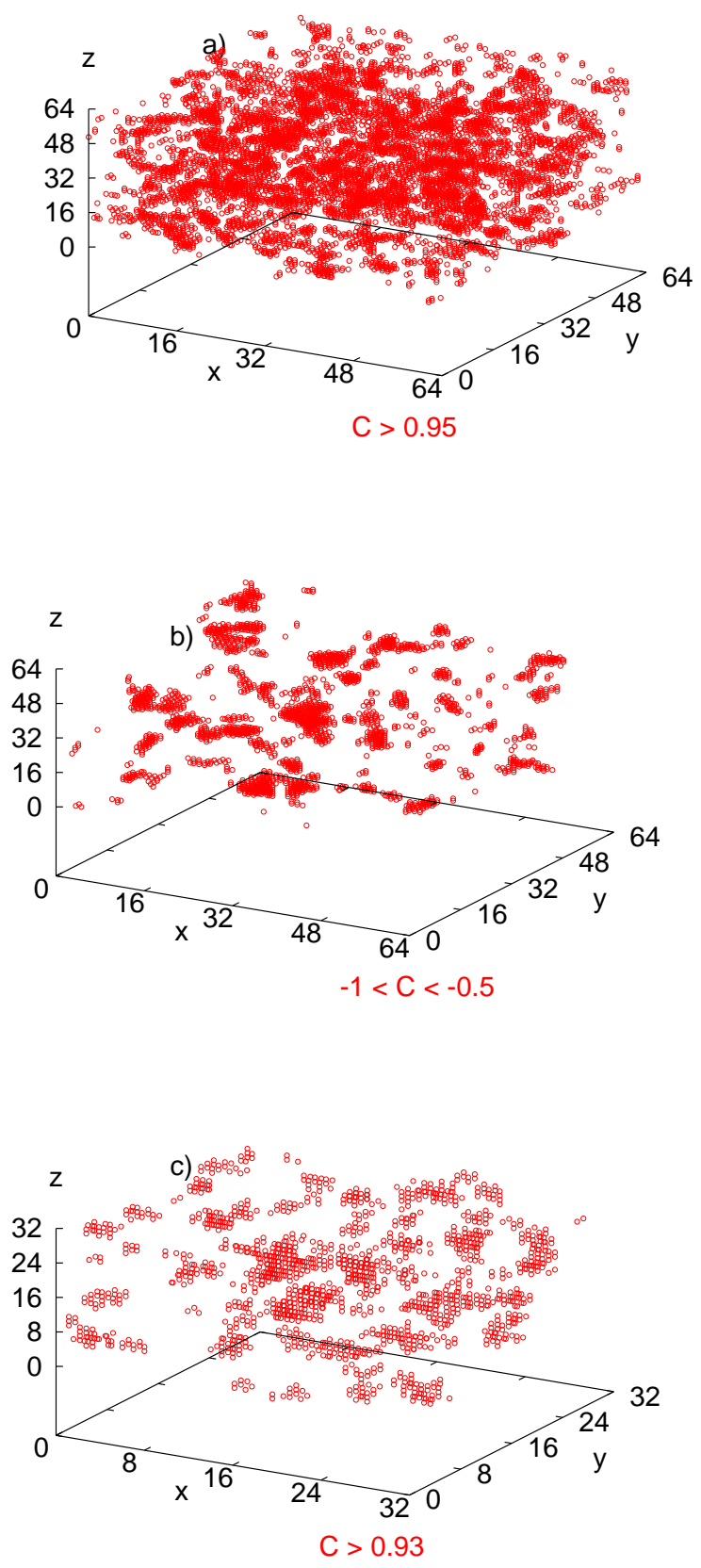


\section{A. Fractal analysis}

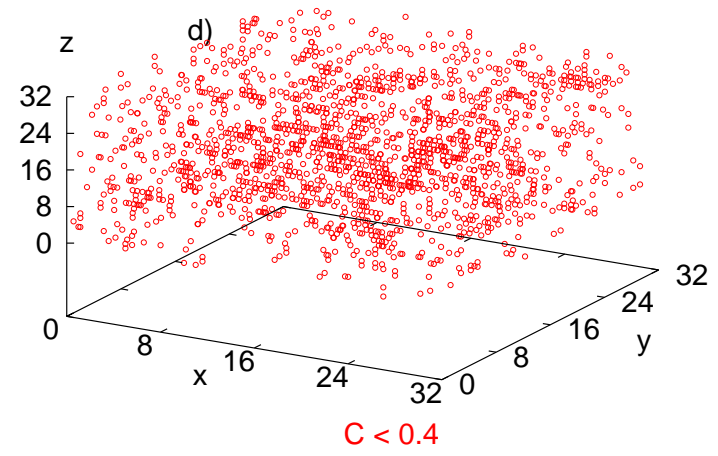

FIG. 52. a) Slices of $C_{i}^{c g} \in[0.95,1]$ (extended) and b) $-1<C_{i}^{c g} \leq-0.5$ (localized). The coarse-graining length is $2 M+1=3 . L=64, t / t_{w}=2, t_{w}=2 \times 10^{4} \mathrm{MCs}, T=0.72 T_{c}$. Slices of c) $C_{i}^{n a} \in[0.93,1]$ (localized) and d) $C_{i}^{n a}<0.4$ (extended), and $L=32$ with $t_{w}=3.2 \times 10^{4} \mathrm{MCs}, t / t_{w}=2$, $\tau=3200 \mathrm{MCs}, T=0.7$.

The richness of the spatial structure is illustrated in Fig. 52a) and Fig. 52b) by highlighting slices of the correlation $C_{i}^{c g}$ taking values in a chosen interval. We show slices with $\mathrm{C}=0.95$ and $d \mathrm{C}=0.05$ in Fig. 52a), and slices of negatively correlated sites in Fig. $52 \mathrm{~b}$ ). The waitingtime is $t_{w}=2 \times 10^{4} \mathrm{MCs}$ and $t / t_{w}=2$. The coarsegraining time is $\tau=10^{3}$ MCs. Regions with negative correlation are well localized in space while the sites with large $C_{i}^{c g}$ are evenly distributed throughout the sample. We find an essentially space-filling distribution of points for any choice of positive $C_{i}^{c g}$ larger than about 0.1 and a localized distribution of points for $C_{i}^{c g} \in[-1,0]$. It should also be noted that there is a distinct spatial anticorrelation between the location of sites with positive and negative correlation. We find that the number of points with $C \leq 0$ scales approximately as $L^{2}$ and that the number of points with $C>0$ scales approximately as $L^{3}$ for both $L=32$ and $L=64$.

When we look at the spatial structure for noiseaveraged correlations, a different picture emerges. In this case, there appear to be space-filling distributions of points for low correlations up to about $C_{i}^{n a} \sim q_{\mathrm{EA}}$ in Fig. 52c) and localized regions of correlations for $C_{i}^{n a}$ close to 1 in Fig. 52d). Indeed, one might have expected that only sites that are essentially forced by the disorder realization to take a particular configuration and do not decay in time (i.e. those with large values of $C_{i}^{n a}$ ) would be likely to show a localized distribution of points. In the noise-averaged case, there are essentially no sites with negative correlations, as can be seen in Fig. 52c)d) (see also Fig. 13 for the PDF of noise-averaged local correlations).
We next studied the fractal dimension of clusters of connected spins with $C_{i}^{c g} \in[\mathrm{C}, \mathrm{C}+d \mathrm{C}]$. The total number of spins in the cluster is regarded as its "mass", $m$, and its radius of gyration, $R_{g}$, is evaluated via the definition $R_{g}^{2}=1 /\left(2 m^{2}\right) \sum_{i j}\left|\vec{r}_{i}-\vec{r}_{j}\right|^{2}$ where $\vec{r}_{i}$ is the position of spin $i$ [74]. The fractal dimension $d_{f}$ of the clusters is defined from the scaling of the "mass" $m$ with the radius of gyration $R_{g}, m \propto R_{g}^{d_{f}}$.

\section{Coarse-grained correlations}

Figure 53 a) shows $m$ against $R_{g}$ for the $3 d$ EA model and five pairs $(\mathrm{C}, d \mathrm{C})$ corresponding to $\mathrm{C}<0,0<\mathrm{C}<q_{\mathrm{EA}}$, $\mathrm{C} \sim q_{\mathrm{EA}}$ and $\mathrm{C}>q_{\mathrm{EA}}$ (see the key). The coarse-graining linear size is $M=1$. There is no qualitative difference between the five sets of data and they are consistent with $d_{f}=2.0 \pm 0.1$. It should be noted, however, that we only fit over two decades in the number of spins and it would be desirable to have a larger dynamic range to get more precise results. We obtained the same results for other values of $t_{w}$ and $t$ and for different temperatures $T<T_{c}$, see panel b) in the same figure. The results are relatively independent of the value of $d \mathrm{C}$, the values of $d \mathrm{C}$ chosen are such that they are big enough that they wash out some of the site-to-site fluctuations due to thermal noise, but also small enough that they do not lump very different values of the correlation in the same bin. The results do not depend that strongly on the coarse-graining volume until one gets to large coarse-graining volumes such as $M=13$, for which $d_{f} \sim 2$ for small clusters with a crossover to $d_{f} \sim d$ at larger cluster sizes.

In Sec. V we defined a two-time dependent correlation length that is of the order of 3 to 4 lattice spacings for the times and temperatures we have considered. Notice that all the clusters obtained using $M=1$ have $R_{g}<3$, which is of the order of the correlation length $\xi$. This is consistent with having $d_{f} \sim 2<d=3$ for $R_{g}<\xi$.

Some recent work suggested that one class of low energy excitations in $3 d$ EA spin glasses have the properties of lattice animals [18] and hence a fractal dimension of 2. In that study, a fractal dimension $d_{f} \sim 2$ was found for the lowest energy excitations above the ground state made of a connected cluster with chosen number of spins and a given site. The precise connection between these objects and the ones studied here is, however, not clear. However, it is intriguing that the fractal dimension observed here is identical to the one they observed. 

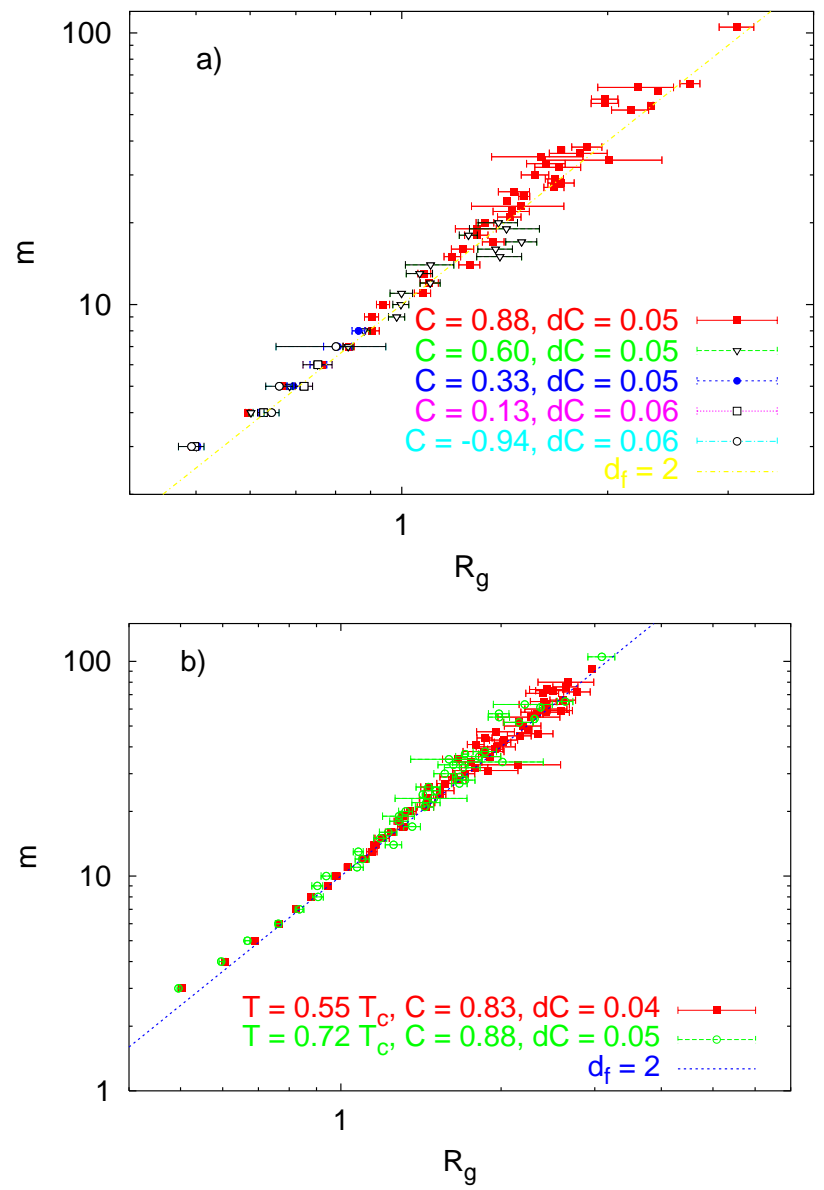

FIG. 53. Number of spins $(m)$ against radius of gyration $\left(R_{g}\right)$ for the coarse-grained correlations in the $3 d$ EA model. $M=1$. Panel a) $T=0.72 T_{c}$ and three pairs of $\mathrm{C}$ and $\mathrm{dC}$. $t_{w}=6.4 \times 10^{5} \mathrm{MCs}$ and $t / t_{w}=2$. Panel b) two values of $T, 0.56 T_{c}\left(t_{w}=3.2 \times 10^{5} \mathrm{MCs}\right.$ and $\left.t / t_{w}=2\right), T=0.72 T_{c}$ $\left(t_{w}=6.4 \times 10^{5} \mathrm{MCs}\right.$ and $\left.t / t_{w}=2\right)$. The dotted lines correspond to $d_{f}=2$.

In $d=2$ the glass transition occurs at $T_{c}=0$. However, the dynamics at low $T$ and large finite times strongly resembles those seen in $3 d$. Several "glassy" features are observed, such as aging phenomena and a non-trivial relation between global correlation and response [69], that eventually disappear at very long times. In Fig. 54 we analyze the fractal dimension of a two dimensional system with $L=128, M=3, t_{w}=5 \times 10^{3}$ MCs and $t / t_{w}=16$. The dotted lines indicate where the points would be expected to lie for $d_{f}=2, d_{f}=1.7$ and $d_{f}=1.5$. Lattice animals in two dimensions have $d_{f} \simeq 1.5$ below the percolation threshold, $d_{f} \simeq 1.85$ at the percolation threshold, and $d_{f} \simeq 2$ for percolating clusters [75]. The results here are not inconsistent with lattice animals in $2 d$, but the large error bars make it hard to definitively establish a connection. (The er- rorbars are much more important than in $d=3$ since we work with many fewer spins: $128^{2}=16384$ as opposed to $64^{3}=262144$.) The ratio $t / t_{w}$ used in the plot is quite large, similar results are obtained for other (smaller or larger) ratios.

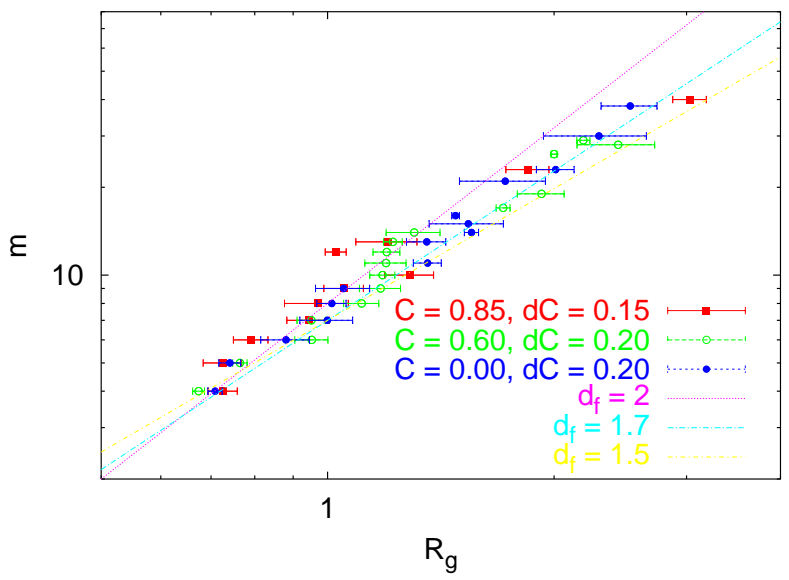

FIG. 54. Number of spins $(m)$ against radius of gyration $\left(R_{g}\right)$ for the coarse-grained correlations in the $2 d$ EA model. $t_{w}=5 \times 10^{3}$ MCs and $t / t_{w}=16 . T=0.8 . M=1$.

\section{Noise averaged correlations}

In addition to looking at the coarse-grained correlations, we also performed a fractal analysis on the noiseaveraged correlations. Whilst there are a number of similarities between the data, there were also some significant differences. At relatively short $t_{w}$ and $t$, essentially all values of $C$ appear to have $d_{f} \simeq 2$, as can be seen in Fig. 55.

However, at longer $t$, or $t_{w}$ there are two types of behavior, depending on the value of the correlation. For correlations $\mathrm{C}$ less than the peak in the distribution of $C_{i}^{n a}$, the fractal dimension is close to 2 , as found in all other cases. However, for large values of the correlation (close to 1 ), it appears that the $d_{f} \sim 2.5$ for small clusters, with a crossover to $d_{f} \sim 2$ at larger cluster sizes. This is illustrated in Fig. 56. This large value of $d_{f}$ suggests that the clusters contributing for these values of the correlation may be those that do not have the effect of domain walls passing through them many times, as they are the same population of spins that are localized in the 3 dimensional plot. 


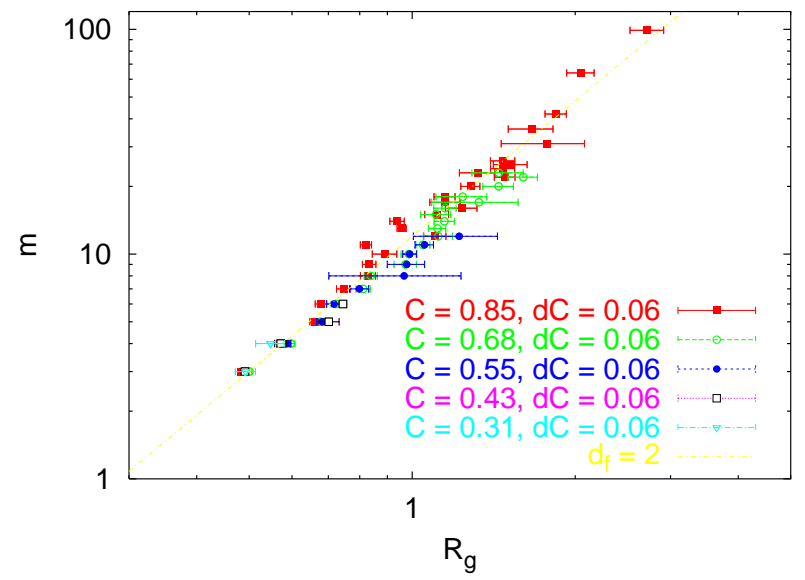

FIG. 55. Number of spins $(m)$ against radius of gyration $\left(R_{g}\right)$ for the noise-averaged correlations in the $3 d$ EA model. $L=32, t_{w}=8 \times 10^{3} \mathrm{MCs}, t=1.28 \times 10^{4} \mathrm{MCs}, T=0.7$ and the averaged has been done using 868 noise realizations for the same random exchanges.

Note that the same phenomenology shown in Fig. 56 was also seen at lower temperatures, $T=0.56 T_{c}$. There is no qualitative difference between the data at that temperature and the data shown here.

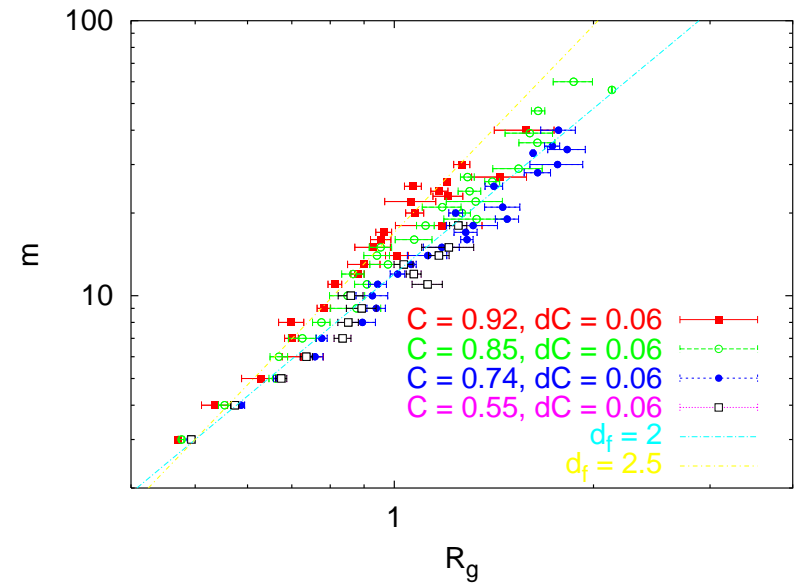

FIG. 56. Number of spins $(m)$ against radius of gyration $\left(R_{g}\right)$ for the $3 d$ EA model. $L=32, t_{w}=3.2 \times 10^{4}, t=48000$, $T=0.7822$ samples.

\section{Average cluster sizes}

Another way to analyze the clusters defined above is to look at their average size for a given value of the correlation. As illustrated in Fig. 57, it is clear that the largest clusters are found in the vicinity of $C_{i}>q_{\mathrm{EA}}$ for both the noise-averaged and coarse-grained cases. This average cluster size for a given correlation $C$ is defined as (note that $m_{k}(C)$ is the "mass" of the $k^{t h}$ cluster at correlation $C$ )

$$
m_{\mathrm{AV}}(C) \equiv \frac{1}{n(C)} \sum_{k=1}^{n(C)} m_{k}
$$

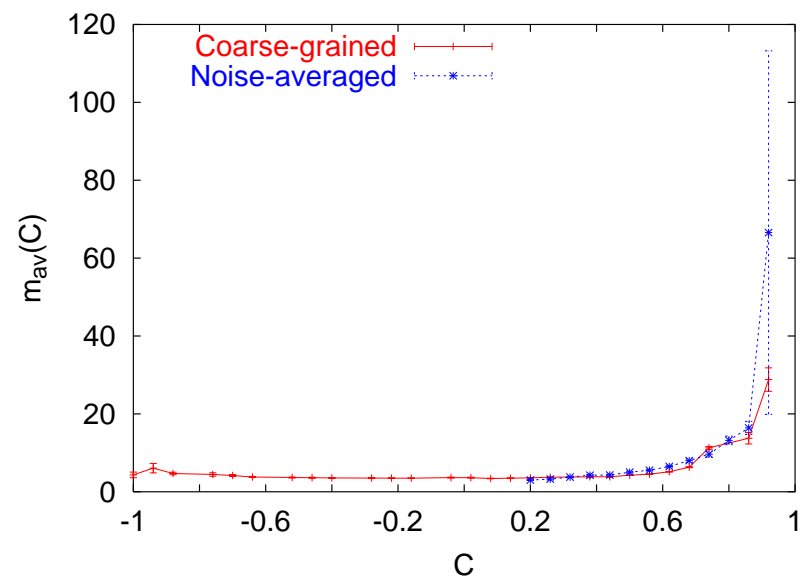

FIG. 57. Average cluster size $m_{\mathrm{Av}}$ against the value of the correlation on the cluster $\mathrm{C}$. $\mathrm{dC}=0.06$. The system size is $L=64, T=0.72 T_{c}, t / t_{w}=2$ and $t_{w}=6.4 \times 10^{5}$ MCs for the coarse-grained data. The noise-averaged data is for $L=32$, $T=0.7, t_{w}=3.2 \times 10^{4} \mathrm{MCs}$ and $t / t_{w}=2$.

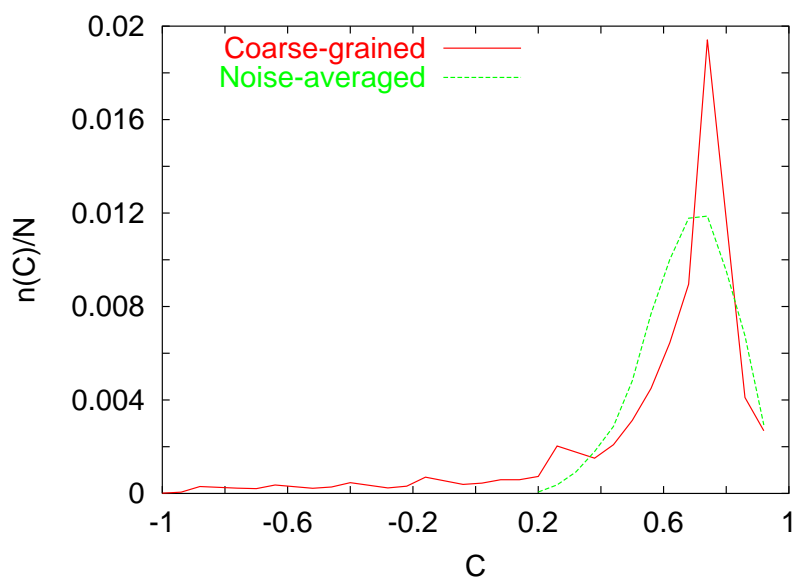

FIG. 58. Number of clusters normalized by the size of the system $N$ as a function of the correlation. Parameters are the same as for Fig. 57.

We also plot the number of clusters at a given correlation, $n(C)$, for both the noise-averaged and coarsegrained cases in Fig. 58. Both have relatively similar forms to the PDF of the correlations, however, in the coarse-grained case there are very few clusters at negative $C$, consistent with the existence of a few localized clusters evident in Fig. 52. The peak in cluster sizes also does not coincide with the peak in $n(C)$, suggesting 
a characteristic state of a large number of moderately small clusters with only a few large clusters even when $\mathrm{C} \sim q_{\mathrm{EA}}$.

\section{B. Multifractal analysis}

Another approach to investigating the spatial structure of correlations in aging dynamics is to perform a multifractal scaling analysis. In a disordered system, different moments of a probability distribution may scale in different ways as the length scale changes. A multifractal analysis seeks to determine the scaling behavior of each moment of the distribution. We do not find any multifractal behavior here, but it would be interesting to look for it in other glassy models or in larger simulations. The method used here, as in the method of fractal analysis, is very simple to implement and could also be used to examine experimental data, such as that collected in confocal microscopy experiments.

We follow a similar procedure to that outlined by Janssen [76], and define a box probability $p_{i}$ such that

$$
p_{i}=\frac{C_{i} \theta\left(C_{i}\right)}{\sum_{j} C_{j} \theta\left(C_{j}\right)}
$$

where $C_{i}$ is taken to be the average correlation in the box. Let the box size be $L_{b}$ and the system size $L$, which leads to a dimensionless parameter $\lambda=L_{b} / L$. The theta function in Eq. (73) is to enforce $p_{i}>0$ and thus $p_{i}$ has an interpretation as a probability. In practice, the theta function is not very important, since the average correlations in the data considered here are positive in all but a very small proportion of the boxes, even at the smallest box size $L_{b}=2$ in 3 dimensions. The distribution of box probabilities $P\left(L_{b}\right)$ is such that when all boxes are included, the total probability is unity. For a given $\lambda$ there are $N(\lambda) \sim \lambda^{-d}$ boxes, where $d$ is the dimension of space. Moments of the distribution are defined via the relation

$$
\left\langle P^{q}\left(L_{b}\right)\right\rangle_{L}=\frac{1}{N(\lambda)} \sum_{i} p_{i}^{q},
$$

and the scaling of these moments is investigated. Multifractal scaling should be valid for

$$
l \ll L_{b}<L \ll \xi,
$$

where $l$ is a microscopic length and $\xi$ is the correlation length, and we expect the moments to scale as

$$
\left\langle P^{q}\left(L_{b}\right)\right\rangle_{L} \sim \lambda^{d+\tau(q)}
$$

where $\tau(q)$ defines a generalized dimension $d(q)$ via $\tau(q)=d(q)(q-1)$ for $q>1$.

\section{3d EA model}

In the $3 d$ EA model it is found that $\tau(2)=3$ and $\tau(3)=6$, corresponding to $d(2)=3$ and $d(3)=3$. The same behavior, i.e. $d(q)=3$ is observed for $q=4,5$, and 6. As is evident in Fig. 59, which is for data with $L=64$, at $T=0.72 T_{c}$ with $t / t_{w}=2,4,16$, and 64 , there appears to be very little dependence on the ratio of the two times.

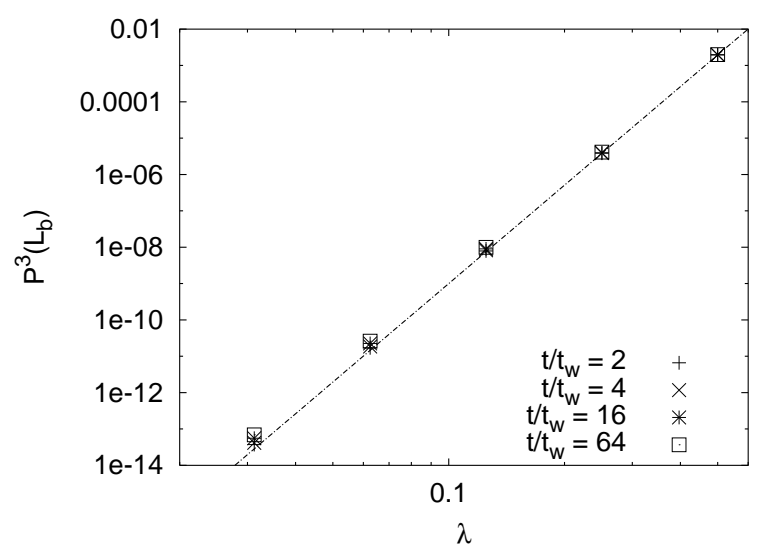

FIG. 59. Multifractal scaling of $P^{3}$ in the $3 d$ EA model. The straight line is a fit to $P^{3}\left(L_{b}\right) \propto \lambda^{9}$, i.e. $d(3)=3$.

\section{2. $2 d$ EA model}

In performing a multifractal analysis of the $2 d$ EA model, the same approach to the analysis was used as outlined for the $3 d$ EA model. The results were similar to the $3 d$ case, in that $d(q) \sim d$ for the times available. In the data shown in Fig. 60, it is clear that for $q=3$, $1.9<d(3)<2$, with perhaps a slightly lower value of $d(q)$ at long waiting times. The data is from a run with $L=128, T=0.8$ and $t_{w}=5000$. The time ratios considered are $t / t_{w}=4,8$ and 16. (Note that alternative definitions of the box probability in $2 d$, such as using $\left|C_{i}\right|$ rather than a theta function can lead to $d(q) \neq d$. In that case it appears to be due to the mixing of non-equilibrium correlations with equilibrium correlations. There will still be such mixing using the definition above, but all negative correlations are excluded, and these may cover a non-trivial number of boxes for small box sizes.) 


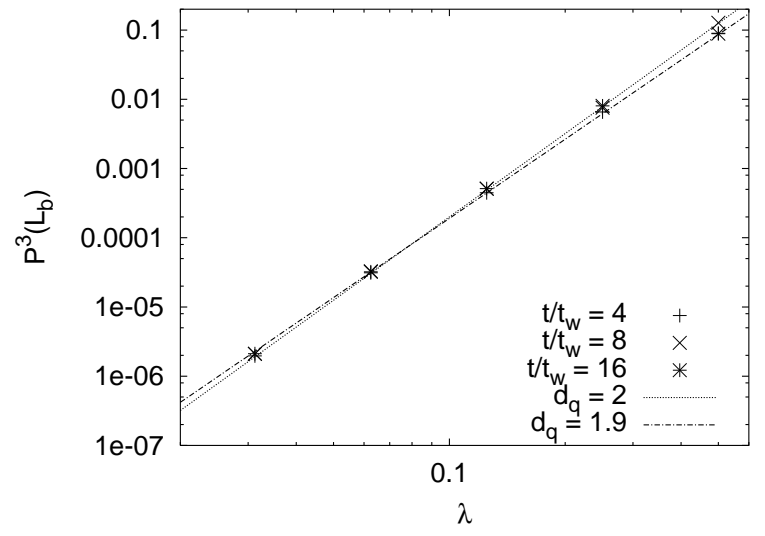

FIG. 60. Multifractal scaling of $P^{2}$ in the $2 d$ EA model. $T=0.8, t_{w}=5 \times 10^{3} \mathrm{MCs}, L=128, t / t_{w}$ as shown in the key.

\section{Summary}

The picture obtained from the above analysis is that the multifractal structure of aging dynamics in 2 and 3 dimensional EA spin glasses is very similar. In both cases, all moments of the probability distribution of correlations appear to scale with the same generalized dimension, which is equal to the dimension of space. The reason why this is so here is that the correlation length $\xi$ is small, of the order 3 to 5 lattice spacings, and therefore we are not truly in the scaling regime defined in Eq. (75). Nonetheless, this method of examining spatial heterogeneities via multifractal analysis may be a useful tool for investigating the behavior observed in different glassy models and in experimental systems.

\section{CONCLUSIONS}

In this paper we showed further evidence that the coarse-grained two-time correlators are the ones that reflect the existence of an asymptotic zero mode in the underlying theory. We defined a two-time correlation length that controls the spatial fluctuations in the coarsegrained local two-time correlators and we showed numerical evidence for the asymptotic divergence of this length in the glassy phase of the $3 d$ EA model, as was to be expected from the existence of the asymptotic zero mode. It should be noted, though, that for the times reached numerically the correlation length is still very short. We argued that in the limit in which the coarsegraining linear size $M$ is taken to diverge together with the correlation length the individual and joint distributions of coarse-grained local correlations and integrated responses should reach a stable form. This means that heterogeneities of all sizes exist in the system. This is another consequence of the asymptotic zero mode.

In disordered systems one can also define noiseaveraged, as opposed to coarse-grained, fluctuating twotime functions. We showed numerically that the fluctuations in these quantities are not controlled by the zero mode and that they behave rather differently than their coarse-grained cousins. In slightly more technical terms, we claim that:

(i) the fluctuations in the local time-reparametrization $h_{i}(t)$ [see Eq. (18)] are coupled to the thermal noise and hence manifest in the fluctuations of the coarse-grained local correlations and responses.

(ii) the fluctuations in the external functions $f_{i}$ [see Eq. (22)], are coupled to the quenched disorder and hence manifest in the fluctuations of the noise-averaged local correlations and responses.

Consequently, in a system with disorder the noiseaveraged local quantities show fluctuations in $f_{i}$ but average out the ones in $h_{i}$. In a system without disorder these quantities do not fluctuate. In contrast, for any coarse-graining volume if the times are long-enough the coarse-grained local quantities keep the fluctuations in $h_{i}$ while the ones in $f_{i}$ are erased since an effective average over the random exchanges is performed.

We related the study of the fluctuations in the local correlations (and susceptibilities) to the study of the evolution of random surfaces. The local two-time functions correspond to the "local heights" of a fluctuating random surface on the $d$ dimensional substrate. We presented a phenomenological effective action for the fluctuations in the local quantities. This allowed us to predict several dynamic properties of their distributions. On the other hand, the geometric analysis of clusters of spins that we introduced in Sec. IX also has a counterpart in the theory of random surfaces; it corresponds to the analysis of contour levels of the surfaces [77]. A complete study of the statistical and dynamic properties of these surfaces might be useful to determine the lower critical dimension of different glassy models.

The analytic calculations that we use as a guideline in this paper where performed using the finite $d$ spin glass Hamiltonian [33]. The numerical data that we present also correspond to this glassy problem. However, we believe that our results are more general and should apply also, with a few modifications, to other glassy problems. In the next section we discuss several possible spin-offs of our results as well as a number of models in which the ideas here discussed can be put to test.

\section{PERSPECTIVES}

Several questions remain open even within the study of the finite $d$ EA model. In particular, we have not checked numerically that a scaling limit is reached by 
taking the limit of long-times and large coarse-graining volumes while keeping $(2 M+1) / \xi\left(t, t_{w}\right)$ fixed. This check remains out of the reach of present computer simulations. Another independent issue that deserves further study is the intriguing fact that the noise-averaged local correlations are quite insensitive to temperature and its possible relation to the observation of memory and rejuvenation in spin-glass experiments. Moreover, the study of the geometric properties of clusters can be improved and made more complete in several directions, e.g. by examining lower temperatures in the $2 d$ case and higher temperatures in the $3 d$ case in order to identify similarities and differences, etc.

We have stated that our approach and its results, derived in this manuscript for spin models with disorder, can be adapted to describe the structural glass problem. Let us summarize what we expect in this case.

Dynamic heterogeneities in the super-cooled liquid phase have been identified numerically $[31,32]$ and experimentally [26-28]. In the case of particle (or molecular) interacting systems the natural and simplest two-time local correlation that makes contact with our approach is the density-density correlator defined on boxes with volume $V_{B}=(2 M+1)^{d}$. Having partitioned the total volume $V$ in $V / V_{B}$ such boxes, one then has $V / V_{B}$ local correlators and local responses (if a perturbation coupled to the density is applied) with which one can construct the PDFs.

In the super-cooled liquid phase we expect that the local correlations and integrated responses defined in boxes of finite size will be typically stationary (after a sufficiently long waiting-time that goes beyond the equilibration time) but with different finite structural relaxation times. This is consistent with the experimental observation that dynamic heterogeneities in supercooled liquids seem to have a lifetime of the order of the relaxation time. At high temperatures the size of the heterogeneities is finite and hence one should suppress the fluctuations by using sufficiently large coarse-graining volumes. The correlation length $\xi\left(t, t_{w}\right)$, that is also stationary, should remain finite, even in the limit of long-times. From a theoretical point of view, this picture is, in a sense, similar to the one that describes the paramagnetic phase in the $O(N)$ model, just above the ordering transition temperature.

When lowering the temperature the size and life-time of the heterogeneities increases [31]. A mean-field-like, or mode-coupling-like approach predicts that their typical size will diverge at the mode coupling transition temperature [78]. We expect then that the correlation length $\xi\left(t, t_{w}\right)$ will saturate at a higher value when $T$ decreases approaching $T_{c}$. In real systems the divergence at $T_{c}$ is smoothed and hence $\xi\left(t, t_{w}\right)$ should not strictly diverge.

At still lower temperatures the bulk quantities age and we expect then to observe heterogeneous aging dynamics of the kind described in this paper, with a two-time de- pendent correlation length for the local fluctuations. The heterogeneities will age too, in a "dynamic" way. By this we mean that if a region looks older than another one when observed on a given time-window, it can reverse its status and look younger than the same other region when observed on a different time-window.

The numerical studies of the global two-time correlations and integrated susceptibilities of Lennard-Jones mixtures $[53,54]$ have shown a remarkable accord with the predictions from the analytic solution to mean-fieldlike glassy models $[1,2]$. We then expect that the PDFs of local correlations and local integrated responses, for the same time-scales used for the bulk calculations, will show the main features described in this paper. In particular, we expect the joint PDFs to reproduce the sketch shown in Fig. 3.

In the glassy phase, we expect the correlation length of structural systems to grow for increasing times roughly in the manner here described. However, this growth might be modified for long enough times when the dynamics crosses over to a different, activated-like, nonequilibrium regime that we cannot characterize theoretically $[1,87]$. We cannot predict what happens to the correlation length on these extremely long time-scales.

The ideas discussed in this paper should not only apply to systems that relax in a nonequilibrium manner as glasses but also to systems that are kept out of equilibrium with a (weak) external forcing. As shown in Ref. [79] the time-reparametrization of the bulk quantities that is selected dynamically is very easy to modify with external perturbations. Indeed, a small force that does not derive from a potential and is applied on every spin in the model renders an aging $p$ spin model stationary [80] while the model maintains a separation of timescales in which the fast scale follows the temperature of the bath, $T$, while the slow scale is controlled by an an effective temperature, $T_{\mathrm{EFF}}>T$. In this case, the aging system selects a time-reparametrization $h(t)=t[9,56]$ while in the perturbed model $h(t)=e^{-t / \tau_{\alpha}}$. Similarly, the aging of a Lennard-Jones mixture is stopped by a homogeneous shear [54]. A different way to modify the time-reparametrization that characterizes the decay of the correlations is by using complex thermal baths [79]. Recently, there has been much interest in the appearance of shear localization, in the form of shear bands, in the rheology of complex fluids [81,82]. Along the lines here described it would be very interesting to analyze the fluctuations in the local reparametrizations in the fluidized shear band and the "jammed" glassy band.

The appearance of an asymptotic invariance under time-reparatrizations in the mean-field dynamic equations was related to the reparametrization invariance of the replica treatment of the statics of the same models $[6,83]$. The latter remains rather abstract. Recently, Brézin and de Dominicis [83] studied the consequences of twisting the reparametrizations in the replica approach. 
Interestingly enough, this can be simply done in a dynamic treatment either by applying shear forces, as discussed in the previous paragraph or by applying different heat-baths to different parts of the system. More precisely, using a model with open boundary conditions one could apply a thermal bath with a characteristic timescale on one end and a different thermal bath with a different characteristic time-scale on the opposite end and see how a time-reparametrization "flow" establishes in the model.

We would like to end this article by mentioning a number of other tests and interesting applications of the ideas here described to other models with a slow relaxation.

(i) In this paper we studied the distributions of the two-time, local in space, spin-spin correlations and their associated responses. In a finite dimensional system one can construct many other two-time correlations that are still local in space. The question then arises as to if all the distributions of all possible correlators have the same qualitative features.

(ii) An important property of the interpretation of the FDR in terms of effective temperatures is that in systems that reach an asymptotic regime with slow dynamics and small entropy production one expects that all observables evolving in the same time-scale partially equilibrate and hence have the same value of the effective temperature [59]. Related to this question one can try to determine if the joint probability distributions of the local correlations and susceptibilities of other pairs of observables are the same and have the same time evolution.

(iii) We expect to see a similar behavior of the local FDR in simple systems undergoing domain growth [44]. More precisely, using the coarse-grained local correlations between the fluctuations in the magnetizations and their associated coarse-grained local susceptibilities, we expect to find a joint PDF that is mostly concentrated along a flat, global $\tilde{\chi}(C)$ curve when $d>1$. This statement can be checked rather easily with Monte Carlo simulations of the finite dimensional ferromagnetic Ising model.

(iv) Related to (iii), the ferromagnetic Ising chain is a particularly interesting case of study. It has been proven that at zero temperature (or when the coupling strength diverges) the global FDR takes a curved form $\tilde{\chi}(C)$ [84]. For this model it might be possible to derive an analytic expression for the joint PDF. Similarly, one can attempt an analytic calculation at criticality in coarsening models as done in Ref. [85].

(v) Kinetically constrained lattice models [42] capture many of the characteristic features of glasses. An analytical study of dynamic heterogeneities in one such spin model has recently appeared [43]. Versions in which one works with particles on a lattice are also rather simple to simulate. In these models one can partition the full lattice in boxes of size $(2 M+1)^{d}$ and define the local two-time density-density correlators within them. A local susceptibility can also be easily defined following
Ref. [86]. A check of the form of the joint probability distribution and its evolution in time is an interesting problem.

(vi) One would like to study realistic models of glass formers with molecular dynamics and test the scaling laws and qualitative features in these cases.

(vii) Herisson and Ocio [58] studied recently the bulk two-time correlation between magnetic fluctuations and the bulk two-time integrated response to an external magnetic field (magnetic susceptibility) of an insulator spin-glass. Their aim was to test the modifications of the global FDT in this nonequilibrium system. In order to have smooth data, they averaged these quantities over many repetitions of the experiment done after heating the sample above the transition. In our terms, the bulk correlations were averaged over different noise-realizations. Another experimental system in which the two-time evolution of a bulk two-time correlation has been recorded, in this case the voltage noise self-correlation, is laponite [30]. Large fluctuations appear. It will be very interesting to analyze the PDFs of the two-time noise-noise correlation and th e two-times integrated response along the lines here described.

Last, but not least, the approach based on reparametrization invariance suggests that it may be possible to search for universality in glassiness. For example, a Ginzburg-Landau theory for phase transitions captures universal properties that are independent of the details of the material. It is symmetry that defines the universality classes. For example, one requires rotational invariance of the Ginzburg-Landau action when describing ferromagnets. Reparametrization invariance may be the underlying symmetry that must be satisfied by the Ginzburg-Landau action of all glasses. What would determine if a system is glassy or not? We are tempted to say the answer is if the symmetry is generated or not at long times. Knowing how to describe the universal behavior may tell us all the common properties of all glasses, but surely it will not allow us to make non-universal predictions, such as what is the glass transition temperature for a certain material, or whether the material displays glassy behavior at all. This quest for universality is a very interesting theoretical scenario that needs to be confronted.

Acknowledgements This work was supported in part by the NSF grants DMR-98-76208 and INT-01-28922 and the Alfred P. Sloan Foundation (C. C.), CNRS-NSF grant, an ACI-France, the Guggenheim Foundation, and ICTP-Trieste (L .F. C.), CONICET and the Universidad Nacional de Mar del Plata, Argentina (J. L. I.). C. C. thanks the LPTHE - Jussieu and M. P. K. thanks BU for their hospitality. The simulations were done with the BU Scientific Supercomputing and Visualization facilities.

We especially thank J. Kurchan for very enlightning 
discussions throughout the development of this work. We also thank L. Balents, A. Barrat, S. Franz, D. Huse, A. Montanari, G. Parisi, L. Radzihovsky, D. Reichmann, and X.-G. Wen for suggestions and discussions.

[1] For a review see L. F. Cugliandolo, cond-mat/0210312, to appear in Slow Relaxations and nonequilibrium dynamics in condensed matter, Les Houches Session 77, J.-L. Barrat, J. Dalibard, M. V. Feigel'man and J. Kurchan eds. (Springer-Verlag, Berlin, 2003).

[2] J.-P. Bouchaud, L. F. Cugliandolo, J. Kurchan, and M. Mézard, in Spin glasses and random fields, A. P. Young ed. (World Scientific, 1998).

[3] G. Parisi, to appear in Slow Relaxations and nonequilibrium dynamics in condensed matter, Les Houches Session 77, J.-L. Barrat, J. Dalibard, M. V. Feigel'man, and J. Kurchan eds. (Springer-Verlag, Berlin, 2003).

[4] K. Binder and A. P. Young, Rev. Mod. Phys. 58, 801 (1986); K. Fischer and J. Hertz, Spin-glasses, (Cambridge Univ. Press, Cambridge, 1991); M. Mézard, G. Parisi and M. A. Virasoro, Spin-glasses and beyond (World Scientific, Singapore, 1987).

[5] V. S. Dotsenko, M. V. Feigel'man, and L. B. Ioffe, Spin Glasses and related Problems (Soviet Scientific Reviews 15) (New York: Harwood Academic, 1990).

[6] H. Sompolinsky and A. Zippelius, Phys. Rev. Lett. 45, 359 (1981); Phys. Rev. B 25, 274 (1982); H. Sompolinsky, Phys. Rev. Lett. 47, 935 (1981).

[7] L. F. Cugliandolo and J. Kurchan, J. Phys. A 27, 5749 (1994).

[8] T. R. Kirkpatrick and D. Thirumalai, Phys. Rev. Lett. 58, 2091 (1987); Phys. Rev. B 36, 5388 (1987); T. R. Kirkpatrick and P. Wolynes, Phys. Rev. B 36, 8552 (1987).

[9] L. F. Cugliandolo and J. Kurchan, Phys. Rev. Lett. 71, 173 (1993).

[10] G. Parisi, Phys. Rev. Lett. 43, 1754 (1979); J. Phys. A 13, 1101 (1980); ibid, 1887 (1980); ibid, L115 (1980).

[11] D. S. Fisher and D. A. Huse, Phys. Rev. Lett. 56, 1601 (1986); Phys. Rev. B 38, 386 (1988).

[12] D. S. Fisher and D. A. Huse, Phys. Rev. B 38, 373 (1988).

[13] W. L. McMillan, Phys. Rev. B 28, 5216 (1983); ibid 31, 340 (1985); A. J. Bray and M. A. Moore, J. Phys. C 17, L463 (1984); and in Heidelberg Colloquium on glassy dynamics, Vol. 275 of Lecture notes in physics, J. L. van Hemmen and I. Morgenstern ed. (Springer, Berlin, 1986). G. J. M. Koper and H. J. Hilhorst, J. Phys. (France) 49, 429 (1988).

[14] J. Villain, Europhys. Lett. 2, 871 (1986).

[15] J. Houdayer and O. C. Martin, Europhys. Lett. 49, 794 (2000); J. Houdayer, F. Krzakala, and O. C. Martin, Eur. J. Phys. B 18, 467 (2000).

[16] F. Krzakala and O. C. Martin, Phys. Rev. Lett. 85, 3013 (2000).

[17] M. Palassini and A. P. Young, Phys. Rev. Lett. 85, 3017
(2000).

[18] J. Lamarcq, J.-P. Bouchaud, O. C. Martin, and M. Mézard, Europhys. Lett. 58, 321 (2002).

[19] H. Bokil, B. Drossel, and M. A. Moore, Phys. Rev. B 62, 946 (2001).

[20] T. Komori, H. Yoshino, and H. Takayama, J. Phys. Soc. Jpn. 68, 3387 (1999); 69, Suppl. A 228 (2000). K. Hukushima, H. Yoshino, , and H. Takayama, Prog. Theor. Phys. Supp. 138, 568 (2000). H. Yoshino, K. Hukushima, and H. Takayama, Phys. Rev. B 66, 064431 (2002).

[21] The aging properties in spin-glasses are reviewed in E. Vincent, J. Hammann, M. Ocio, J.-P. Bouchaud, and L. F. Cugliandolo, Slow dynamics and aging in glassy systems, in Sitges 1996, ed. M. Rubí (Springer-Verlag, 1997) and [22].

[22] P. Nordblad and P. Svedlindh, Experiments on spinglasses in Spin-glasses and random fields, A. P. Young ed. (World Scientific, 1998).

[23] J.-P. Bouchaud, V. Dupuis, J. Hammann, and E. Vincent, Phys. Rev. B 65024439 (2002).

[24] L. Berthier and J.-P. Bouchaud, Phys Rev. B 66, 054404 (2002).

[25] A recent review on the main experimental features of supercooled liquids and glasses is M. D. Ediger, C. A. Angell, and S. R. Nagel, J. Phys. Chem. 100, 14200 (2000). C. A. Angell, K. L. Ngai, G. B. McKenna, P. F. McMillan, and S. W. Martin, J. Appl. Phys. 88, 3113 (2000).

[26] H. Sillescu, J. Non-Crystal. Solids 243, 81 (1999); M. D. Ediger, Annu. Rev. Phys. Chem. 51, 99 (2000).

[27] E. Vidal-Russell and N. E. Israeloff, Nature 408, 695 (2000).

[28] A. van Blaaderen and P. Wiltzius, Science 270, 1177 (1995); W. K. Kegel and A. van Blaaderen, Science 287, 290 (2000); E. R. Weeks and D. A. Weitz, Phys. Rev. Lett. 89, 095704 (2000); E. R. Weeks, J. C. Crocker, A. C. Levitt, A. Schofield, and D. A. Weitz, Science 287, 627 (2000).

[29] The aging properties of a colloidal glass have been studied with the confocal microscopy technique in R. E. Courland and E. R. Weeks, cond-mat/0209148.

[30] L. Buisson, L. Bocquet and S. Ciliberto, condmat/0210490, Proceedings of "III workshop on non equilibrium phenomena" (Pisa 2002).

[31] C. Bennemann, C. Donati, J. Baschnagel, and S. C. Glotzer, Nature, 399, 246 (1999); W. Kob, C. Donati, S. J. Plimpton, P. H. Poole, and S. C. Glotzer, Phys. Rev. Lett. 79, 2827 (1997).

[32] D. Perera and P. Harrowell, Phys. Rev. E 54, 1652 (1996). C. Donati et al Phys. Rev. E 60, 3107 (1999). A. Onuki and Y. Yamamoto, J. Non-Crystal. Solids 235-237, 19 (1998). B. Doliwa and A. Heuer, condmat/0109317. A. Heuer, M. Kunow, M. Vogel, R. D. Banhatti, cond-mat/0209361.

[33] C. Chamon, M. P. Kennett, H. E. Castillo, and L. F. Cugliandolo, Phys. Rev. Lett. 89, 217201 (2002).

[34] H. E. Castillo, C. Chamon, L. F. Cugliandolo, and M. P. Kennett, Phys. Rev. Lett. 88, 237201 (2002).

[35] P. H. Poole, S. C. Glotzer, A. Coniglio, and N. Jan, Phys. Rev. Lett. 78, 3394 (1997); S. C. Glotzer, N. Jan, and P. Poole, Phys. Rev. E 57, 7350 (1998). 
[36] A. Barrat and R. Zecchina, Phys. Rev. E 59, R1299 (1999).

[37] F. Ricci-Tersenghi and R. Zecchina, Phys. Rev. E 62, R7567 (2000).

[38] A. Montanari and F. Ricci-Tersenghi, cond-mat/0207416.

[39] C. Brangian and W. Kob, J. Phys. A 35, 191 (2002).

[40] R. B. Griffiths, Phys. Rev. Lett. 23, 17 (1969); A. J. Bray and M. A. Moore, J. Phys. C 15, L765 (1982); A. J. Bray, Phys. Rev. Lett. 59, 586 (1987).

[41] J.P. Bouchaud and M. Mézard; J. Phys. I (France) 4, 1109 (1994); E. Marinari, G. Parisi, and F. Ritort; J. Phys. A 27, 7615 (1994); ibid, 7647 (1994); L. F. Cugliandolo, J. Kurchan, G. Parisi, and F. Ritort, Phys. Rev. Lett. 74, 1012 (1995); P. Chandra, L. B. Ioffe, and D. Sherrington, Phys. Rev. Lett. 75, 713 (1996); P. Chandra, M. V. Feigel'man, L. B. Ioffe, and D. M. Kagan, Phys. Rev. B 56, 11553 (1997). G. Franzese and A. Coniglio, Phys. Rev. E 58, 2753 (1998); Phil. Mag. B 79, 1807 (1999).

[42] For a review on kinetically constrained glassy models see F. Ritort and P. Sollich, cond-mat/0210382.

[43] J.-P. Garrahan and D. Chandler, Phys. Rev. Lett. 89, 035704 (2002).

[44] A. J. Bray, Adv. Phys. 43, 357 (1994).

[45] See for example, P. M. Chaikin and T. C. Lubensky, Principles of Condensed Matter Systems (Cambridge University Press, 1995).

[46] G. Parisi, cond-mat/0208070.

[47] The static properties of disordered models have been studied using the cavity method in M. Mézard, G. Parisi, and M. A. Virasoro, Europhys. Lett. 1, 77 (1985); M. Mézard and G. Parisi, cond-mat/0207121; M. Mézard, G. Parisi, and R. Zecchina, Science 297, 812 (2002).

[48] M. P. Kennett and C. Chamon, Phys. Rev. Lett. 86, 1622 (2001); M. P. Kennett, C. Chamon, and J. Ye, Phys. Rev. B 64, 224408 (2001).

[49] L. F. Cugliandolo, J. Kurchan, and P. Le Doussal, Phys. Rev. Lett. 76, 2390 (1996).

[50] S. Franz and M. Mézard, Europhys. Lett. 26, 209 (1994); Physica A (Amsterdam) 210, 48 (1994).

[51] M. Picco, F. Ricci-Tersenghi, and F. Ritort, Eur. Phys. J. B 21, 211 (2001); D. A. Stariolo, M. A. Montemurro, and F. A. Tamarit, cond-mat/0207105.

[52] A summary of numerical studies of the nonequilibrium dynamics in spin models appeared in H. Rieger, Annu. Rev. Comp. Phys. II, 295 (World Scientific, Singapore, 1995). Studies of frustrated lattice gases have been published in: A. Fierro, A. de Candia, and A. Coniglio, Phys. Rev. E 62, 7715 (2002); M. Sellitto, Eur. Phys. J. B 4, 135 (1998); J. J. Arenzon, F. Ricci-Tersenghi, and D. A. Stariolo, Phys. Rev. E 62, 5978 (2000). Moecular dynamic simulations of interacting particle systems can be found in Ref. [53,54].

[53] W. Kob and J.-L. Barrat, Phys. Rev. Lett. 78, 4581 (1997); Eur. Phys. J. B 13, 319 (2000). R. di Leonardo et al, Phys. Rev. Lett. 84, 6054 (2000).

[54] L. Berthier and J.-L. Barrat, Phys. Rev. Lett. 89, 095702 (2002); J. Chem. Phys. 116, 6228 (2002).

[55] The existence of a well-defined plateau for the $3 d \mathrm{EA}$ model is not established beyond any doubt. We shall use it as a hypothesis admitting the possibility that future generation simulations might show that $q_{\mathrm{EA}} \rightarrow 0$ even in $d=3$.

[56] B. Kim and A. Latz, Europhys. Lett. 53, 660 (2001).

[57] S. Franz and H. Rieger, J. Stat. Phys. 79, 749 (1995); E. Marinari, G. Parisi, F. Ricci-Tersenghi, and J. J. RuizLorenzo, J. Phys. A 31, 2611 (1998).

[58] D. Herisson and M. Ocio, Phys. Rev. Lett. 88, 257202 (2002).

[59] L. F. Cugliandolo, J. Kurchan, and L. Peliti, Phys. Rev. E 55, 3898 (1997).

[60] L. F. Cugliandolo, D. S. Dean, and J. Kurchan, Phys. Rev. Lett. 79, 2168 (1997).

[61] H. Sompolinsky and A. Zippelius, Phys. Rev. Lett. 53, 1297 (1983).

[62] S. L. Ginzburg, Zh. Eksp. Teor. Fiz. 90, 754 (1986) [Sov. Phys. JETP 63, 439 (1986)].

[63] L. B. Ioffe, Phys. Rev. B 38, 5181 (1988).

[64] H. Sompolinsky and A. Zippelius, Phys. Rev. Lett. 45, 359 (1981); Phys. Rev. B 25, 6860 (1982).

[65] The dynamic equations are derived in the limit $N \rightarrow \infty$. In this case, the dynamic correlations and responses are self-averaging and independent of the nosie realization. For this reason we do not worry about having to average the two-time quantities in Eqs. (38) and (39).

[66] We shall not consider the possibility of there being several (hierarchical) length-scales. Moreover, to keep the discussion simple as elsewhere in this article, we study the asymptotic properties of the mass keeping $t / t_{w}$ fixed [using a ratio $h(t) / h\left(t_{w}\right)$ fixed might be more precise].

[67] D. A. Huse, Phys. Rev. B 43, 8673 (1991); J. Kisker, L. Santen, M. Schreckenberg, and H. Rieger, Phys. Rev. B 53, 6418 (1996); E. Marinari, G. Parisi, F. RicciTersenghi, and J. J. Ruiz-Lorenzo, J. Phys. A 33, 2373 (2000).

[68] V. Dupuis, E. Vincent, J-P Bouchaud, J. Hammann, A. Ito and H. Aruga Katori, Phys. Rev. B 64, 174204 (2001). P. E. Jönsson, H. Yoshino, P. Nordblad, H. Aruga Katori, and A. Ito, cond-mat/0112389.

[69] A. Barrat and L. Berthier, Phys. Rev. Lett. 87, 087204 (2001).

[70] N. D. Mackenzie and A. P. Young, Phys. Rev. Lett. 49, 301 (1982); J. Phys. C 16, 5321 (1983). A. Baldassarri, cond-mat/9607162, Phys. Rev. E 58, 7047 (1998).

[71] A. J. Bray and M. A. Moore. J. Phys. C 13, 419 (1980).

[72] G. Ferraro, tesi de Laurea, Roma I, 1994. A. Baldassarri, L. F. Cugliandolo, J. Kurchan, and G. Parisi J. Phys. A 28, 1831 (1995).

[73] Some articles in which the dynamics of the SK has been studied numerically are: H. Eissfeller and M. Opper, Phys. Rev. Lett. 68, 2094 (1992); A. Scharnagl, M. Opper and W. Kinzel, J. Phys. A 28, 5721 (1995). H. Yoshino, K. Hukushima, and H. Takayama, Prog. Theor. Phys. Supp. 126 (1997). A. Billoire and E. Marinari, cond-mat/0101177, H. Takayama, H. Yoshino, and K. Hukushima cond-mat/9612071. K. Hukushima and K. Nemoto, J. Phys. Soc. Japan 65, 1604 (1996).

[74] T. Nakayama, K. Yakubo, and R. L. Orbach, Rev. Mod. Phys. 66, 381 (1994).

[75] D. Stauffer, Phys. Rev. Lett. 41, 1333 (1978); R. C. Ball and J. R. Lee, J. Phys. I 6, 356 (1996). 
[76] M. Janssen, Int J. Mod. Phys. B 8, 943 (1994).

[77] J. Kondev, Phys. Rev. Lett. 78, 4320 (1997); C. Zeng, J. Kondev, D. McNamara and A. A. Middleton, Phys. Rev. Lett 80, 109 (1998); J Kondev, C. L. Henley and D. G. Salinas, Phys. Rev. E 61, 104 (2000); M. Schwartz, cond-mat/0012105.

[78] S. Franz and G. Parisi, J. Phys. I 5, 1401 (1995), Phys. Rev. Lett. 79, 2486 (1997). C. Donati, S. Franz, G. Parisi and S. C. Glotzer, Phil. Mag. B 79, 1827 (1999).

[79] L. F. Cugliandolo and J. Kurchan, Physica A 263242 (1999).

[80] L. F. Cugliandolo, J. Kurchan, P. Le Doussal, and L. Peliti, Phys. Rev. Lett. 78, 350 (1997); L. Berthier, J.-L. Barrat, and J. Kurchan, Phys. Rev. E 61, 5464 (2000); L. Berthier, cond-mat/0209394.

[81] L. J. Chen, B. J. Ackerson, and C. F. Zulowski, J. Rheol. 38, 193 (1993). F. Pignon, A. Magnin, and J-M Piau, J. Rheol. 40, 573 (1996). W. Losset, L. Bocquet, T. C. Lubensky, and J. P. Gollub, Phys. Rev. Lett. 85, 1428 (2000). C. Debregead, H. Tabuteau, and J-M di Meglio, Phys. Rev. Lett. 87, 178305 (2001). P. Coussot et al, Phys. Rev. Lett. 88, 218301 (2002). G. Picard, A. Ajdari, L. Bocquet, and F. Lequeux, cond-mat/0206260.

[82] F. Varnik, L. Bocquet, J-l Barratand L. Berthier, condmat/0208485.

[83] C. De Dominicis and E. Brézin, cond-mat/0201066.

[84] C. Godrèche and J.-M. Luck, J. Phys. A 33, 1151 (2000); E. Lippiello and M. Zannetti, Phys. Rev. E 61, 3369 (2000); F. Corberi, E. Lippiello, and M. Zannetti, Phys. Rev. E 63, 061506 (2001); Eur. Phys. J. B 24, 359 (2000); F. Corberi, C. Castellano, E. Lippiello, and M. Zannetti, Phys. Rev. E. (to appear); P. Sollich, S. Fielding, and P. Mayer, cond-mat/0111241 to appear in J. Phys. C.

[85] C. Godrèche and J.-M. Luck, J. Phys. A 33, 9141 (2000); M. Henkel, M. Piemling, C. Godrèche, and J.-M. Luck, Phys. Rev. Lett. 87, 265701 (2001); A. Picone and M. Henkel, J. Phys. A 35, 5572 (2002); M. Henkel, hep-th/0205256; P. Calabrese and A. Gambassi, Phys. Rev. E 65, 066120 (2002); cond-mat/0207452; condmat/0207487.

[86] M. Sellitto, Europ. J. Phys. B 4, 135 (1998).

[87] A solvable model with "mean-field-like" nonequilibrium regime and a slower still nonequilibrium regime has recently been exhibited, see G. Semerjian and L. F. Cugliandolo, cond-mat/0204613, Europhys. Lett. (to appear). 